\title{
Design of a Kilogram Scale, Plug Flow Photoreactor Enabled by High Power LEDs
}

François Lévesque*, Michael J. Di Maso*, Karthik Narsimhan, Michael K. Wismer, John

\author{
R. Naber
}

Process Research and Development, Merck Research Laboratories, Rahway, New Jersey 07065, United States. 


\section{Contents}

1. Pressure Drop of Previous Flow Photochemical Reactor ........................................................3

2. Determination of Extinction Coefficient of Reaction Mixture and Penetration Depth .............4

3. Absorbance and Penetration Depth vs. Conversion \& Selection of Wavelength......................5

4. Calculation of Emitted Photon Equivalence......................................................................8

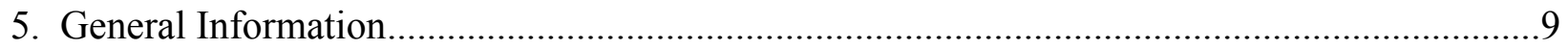

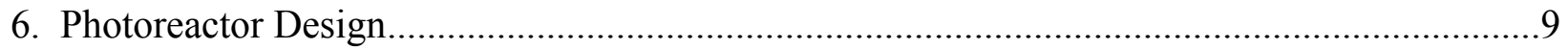

7. LED Plate Output Power Density, Energy Efficiency, and Stability ..................................11

8. Design of Experiments (DoE) with Time Profiles .......................................................13

9. Procedure for the Multi-Kilogram Scale Flow Reaction ....................................................25

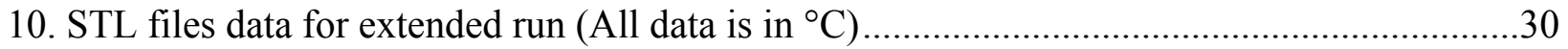


1. Pressure Drop of Previous Flow Photochemical Reactor

$$
\Delta P=\frac{128 \mu Q L}{\pi D^{4}}
$$

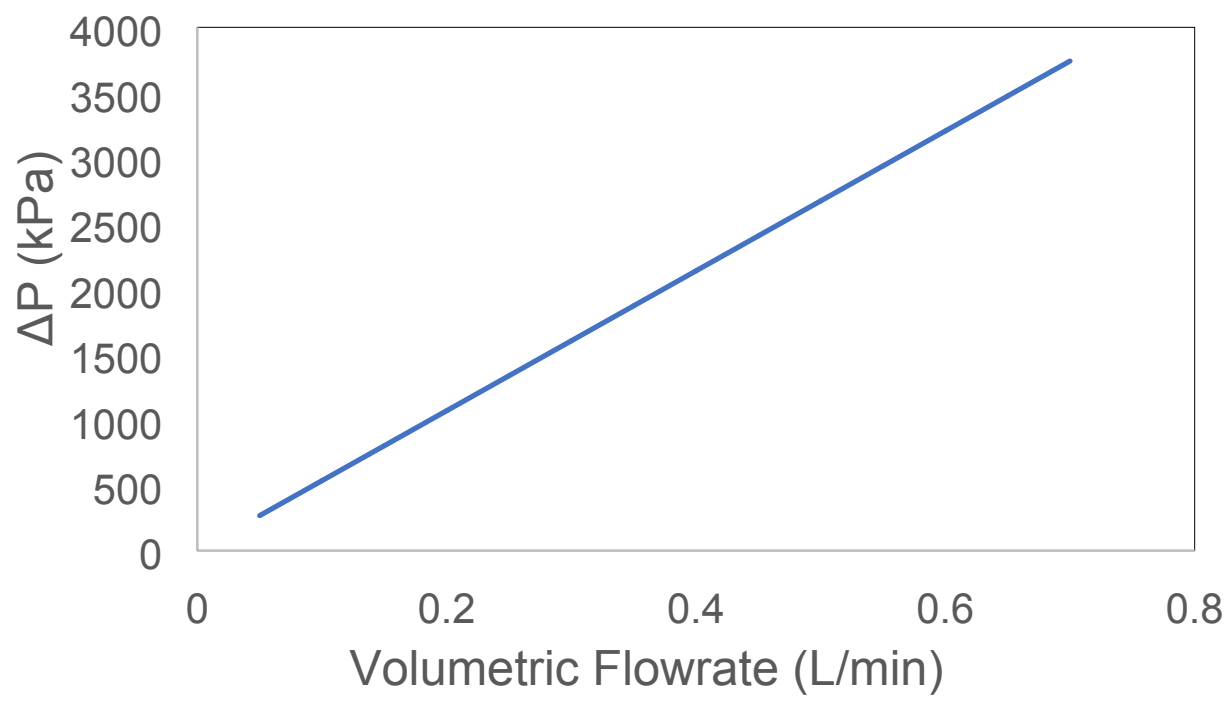

Figure S1: Pressure drop of previous Merck flow photoreactor. Pressure drop was calculated using the Darcy-Weisbach Equation. $\mathrm{Re}<\mathbf{2 3 0 0}$ for all volumetric flow rates examined. 


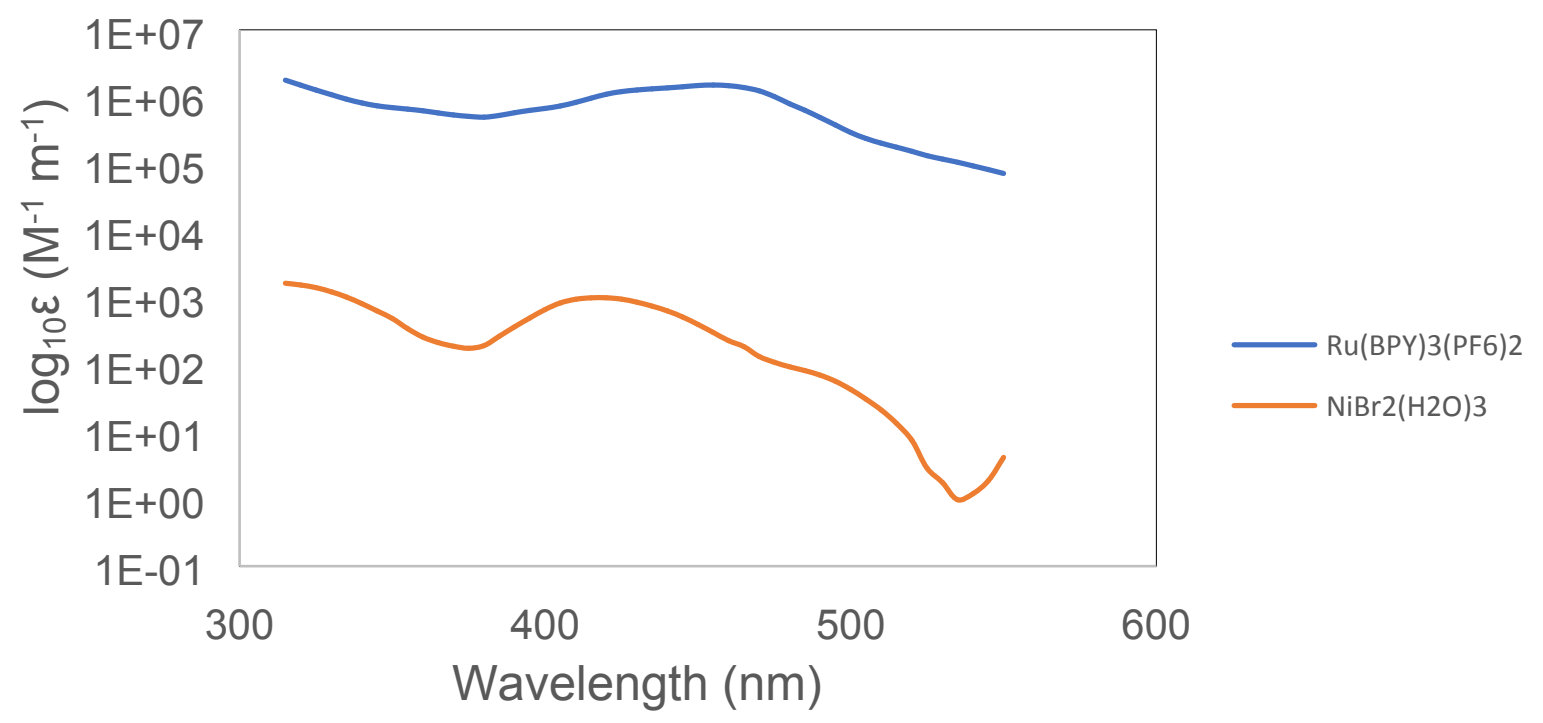

Figure S2: Molar extinction coefficient of $\mathrm{Ru}(\mathrm{BPY})_{3}\left(\mathrm{PF}_{6}\right)_{2}$ and $\mathrm{NiBr}_{2}\left(\mathrm{H}_{2} \mathrm{O}\right)_{3}$ in $\mathrm{DMSO}$. Spectra were collected in Molecular Devices Spectramax 384 Plus UV-visible spectraphotometer in quartz cells of pathlength 1, 2, 3, 5, and $10 \mathrm{~mm}$.

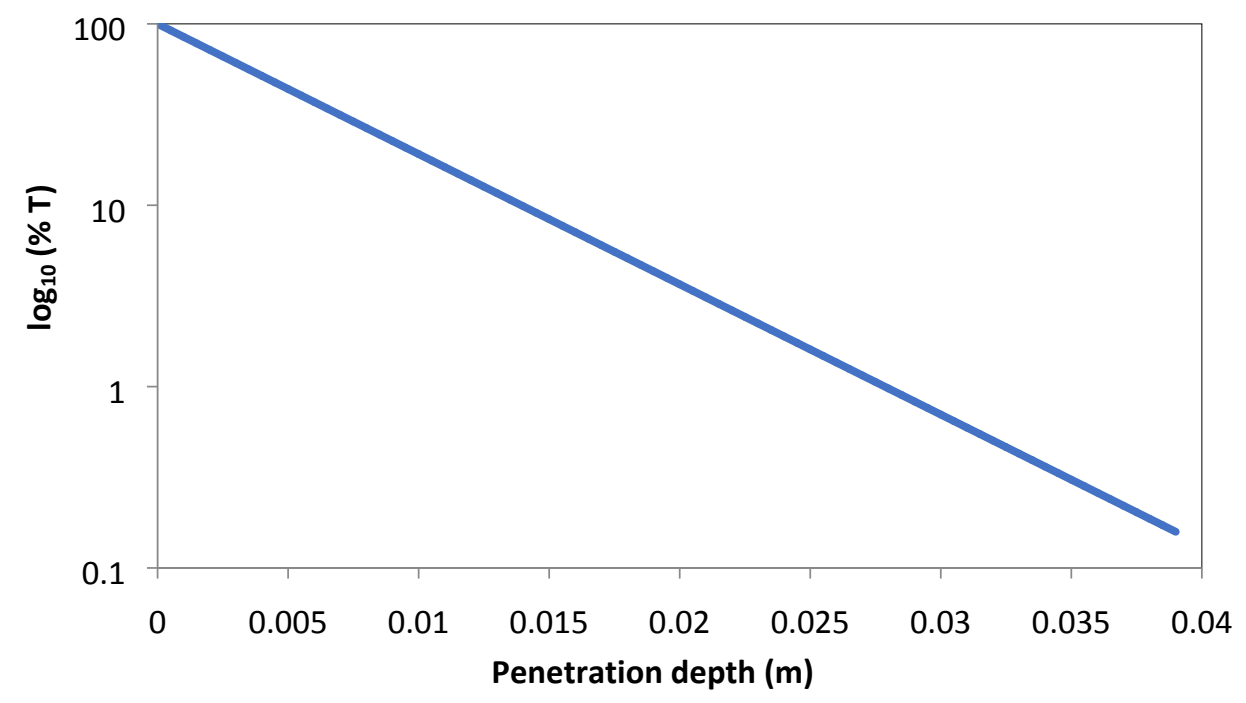

Figure S3: Penetration depth of the photoredox C-N cross coupling reaction in the $250 \mathrm{~mL}$ batch reactor. Reaction conditions: $0.25 \mathrm{M}$ 4-bromobenzotrifluoride, 1.2 eq pyrrolidine, $1.5 \mathrm{eq} \mathrm{1,4-}$ diazabicylco[2,2,2]octane, $3 \mathrm{~mol} \% \mathrm{NiBr}_{2}\left(\mathrm{H}_{2} \mathrm{O}\right)_{3}, 0.02 \mathrm{~mol} \% \mathrm{Ru}(\mathrm{BPY})_{3}\left(\mathrm{PF}_{6}\right)_{2}, \lambda=450 \mathrm{~nm}$. Full absorption of photons was defined at $1 \%$ transmission and was achieved at $0.028 \mathrm{~m}$. Vessel inner diameter was $0.08 \mathrm{~m}$, indicating dark zones in the vessel interior. The reaction was run under conditions of full absorption of photons. 


\section{Absorbance and Penetration Depth vs. Conversion \& Selection of Wavelength}

The wavelength of the cross-coupled amination was optimized by assaying reaction samples for conversion and UV-vis absorption spectra. UV-vis spectroscopy of the neat $\mathrm{NiBr}_{2}$, $\mathrm{Ru}(\mathrm{BPY})_{3}\left(\mathrm{PF}_{6}\right)_{2}$ (Figure S5Error! Reference source not found.) and reaction mixtures showed the electronic transitions of the catalysts changed for conversion above $50 \%$ and corresponded to a decrease in the reaction rate (Figure S4). At short times $(X<50 \%)$, there were strong absorption bands between $250-350 \mathrm{~nm}$ while there were relatively small changes in the visible region of 350-550 nm. The strong peaks at $250-350 \mathrm{~nm}$ appeared even at low conversion (10\%) implying those did not correspond to any products. There were small increases in peaks at 370, 390,430 , and $450 \mathrm{~nm}$ (Figure S4, top row) corresponding to $\mathrm{Ru}(\mathrm{BPY})_{2}\left(\mathrm{PF}_{6}\right)_{2}$ (Figure S5, left). As conversion increased past $50 \%$, the peaks at 370 and $390 \mathrm{~nm}$ dramatically increased while the signatures at 430 and $450 \mathrm{~nm}$ slightly decreased (Figure S4, bottom row). This indicated that solution darkening was minimal in the wavelength range of $430-450 \mathrm{~nm}$.

$$
A(\lambda)=\varepsilon_{e f f}(\lambda) l=\sum_{i=1}^{n} \varepsilon_{i}(\lambda) C_{i} l
$$

The extent of solution darkening was modeled as the "effective" molar extinction coefficient. $\varepsilon_{\text {eff }}$ of the reaction solution was modeled as the sum of all absorbing species at a given wavelength $\lambda . \quad \varepsilon_{\text {eff }}(\lambda, X)$ indicated the absorbtivity of the reaction mixture over the course of the reaction. Based on the increase in absorbance of the reaction for $\lambda<400 \mathrm{~nm}$, these wavelengths were unsuitable to be used for scale-up due to potential of introducing dark zones and mass transfer limitations at large tube diameters. Comparing absorptivity at 405 and 440 $\mathrm{nm}$, two wavelengths commercially available for LEDs, indicated that $405 \mathrm{~nm}$ exhibited an increase in $\varepsilon_{\text {eff }}$ while $440 \mathrm{~nm}$ had relatively constant absorptivity (Error! Reference source not found., left). Thus, at $440 \mathrm{~nm}$, the penetration depth of light into the reaction solution varied between 15-24 mm (Error! Reference source not found., right).

Based on the $\lambda_{\max }$ of $\mathrm{Ru}(\mathrm{BPY})_{2}\left(\mathrm{PF}_{6}\right)_{2}$ between $440-460 \mathrm{~nm}$ (Figure S5), minimal change in absorptivity of the reaction solution at $440 \mathrm{~nm}$ (Figure S6, left), and commercial availablilty of LEDs at the chosen wavelength, the $440 \mathrm{~nm}$ was chosen as the optimum wavelength for reaction scale-up. 

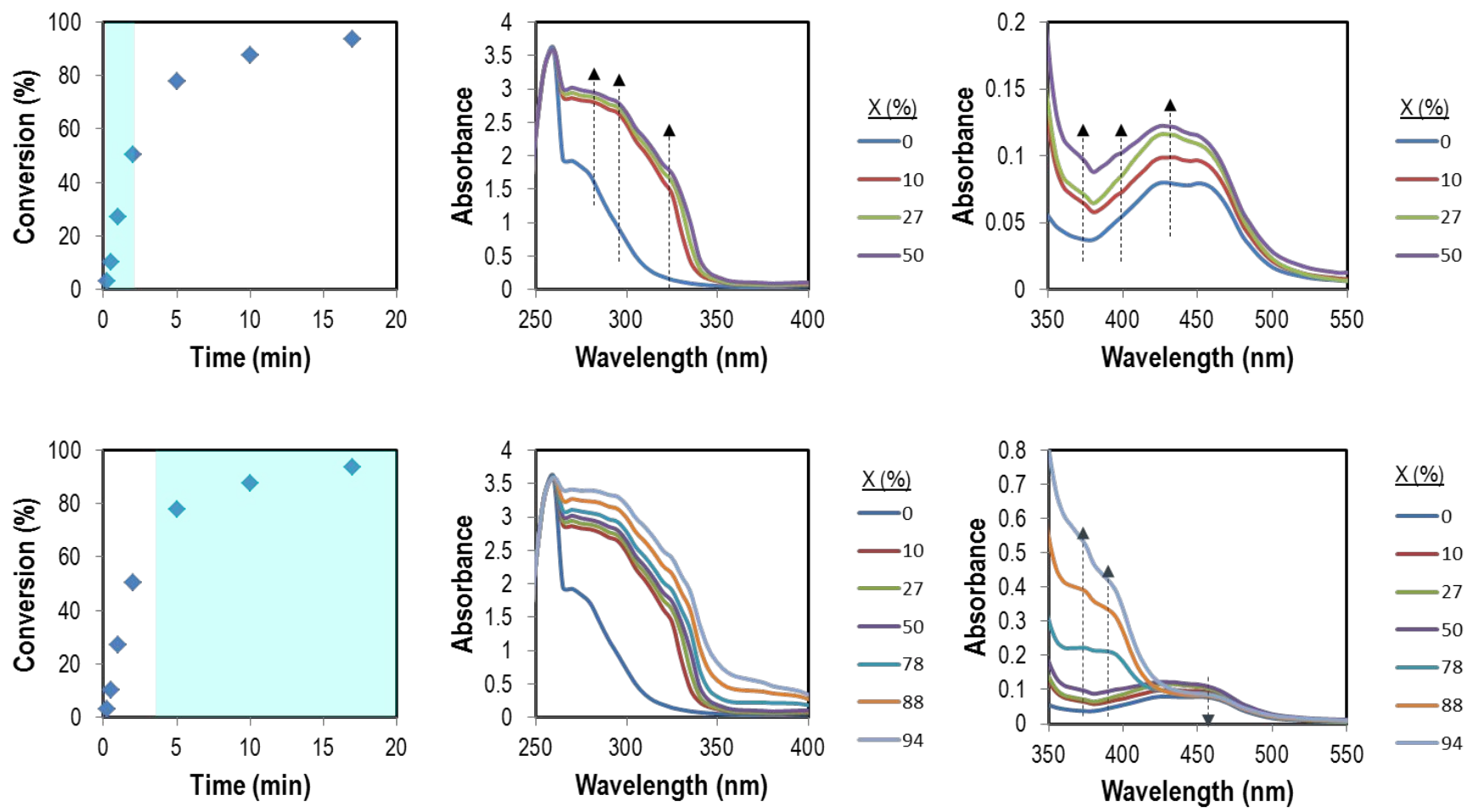

Figure S4: Conversion and UV-visible spectroscopy of time profile samples. Top row is at short reaction times (low conversion) while bottom row is at long reaction times (high conversion). (Left column) Conversion profile. (Middle column) UV-vis spectra of samples from 250-400 nm. (Right column) UV-vis spectra of samples from $350-550 \mathrm{~nm}$. Reaction conditions: $0.25 \mathrm{M}$ trifluorobromobenzene, 1.5 eq pyrrolidine, $1.8 \mathrm{eq}$ DABCO, $0.02 \mathrm{~mol} \% \mathrm{Ru}(\mathrm{BPY}) 3(\mathrm{PF} 6) 2,3 \% \mathrm{NiBr2(H2O)3}$ in DMSO. $3 \mathrm{~mL}$ reaction vol. $\lambda=405 \mathrm{~nm}$. Reference spectra was neat DMSO. Samples were covered in foil when removed from the reactor and before spectra were taken.
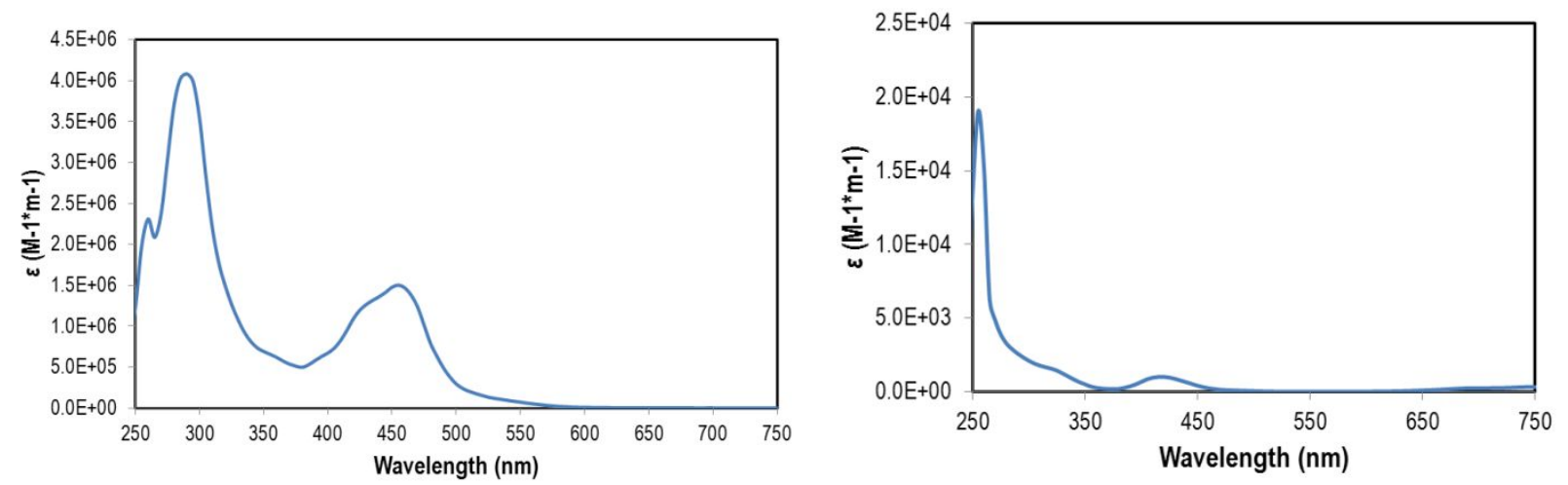

Figure S5: Molar extinction coefficients of (Left) $\mathrm{Ru}(\mathrm{BPY})_{3}\left(\mathrm{PF}_{6}\right)_{2}$ and (Right) $\mathrm{NiBr}_{2}\left(\mathrm{H}_{2} \mathrm{O}\right)_{3}$ in $\mathrm{DMSO}$. Reference spectra was neat DMSO 

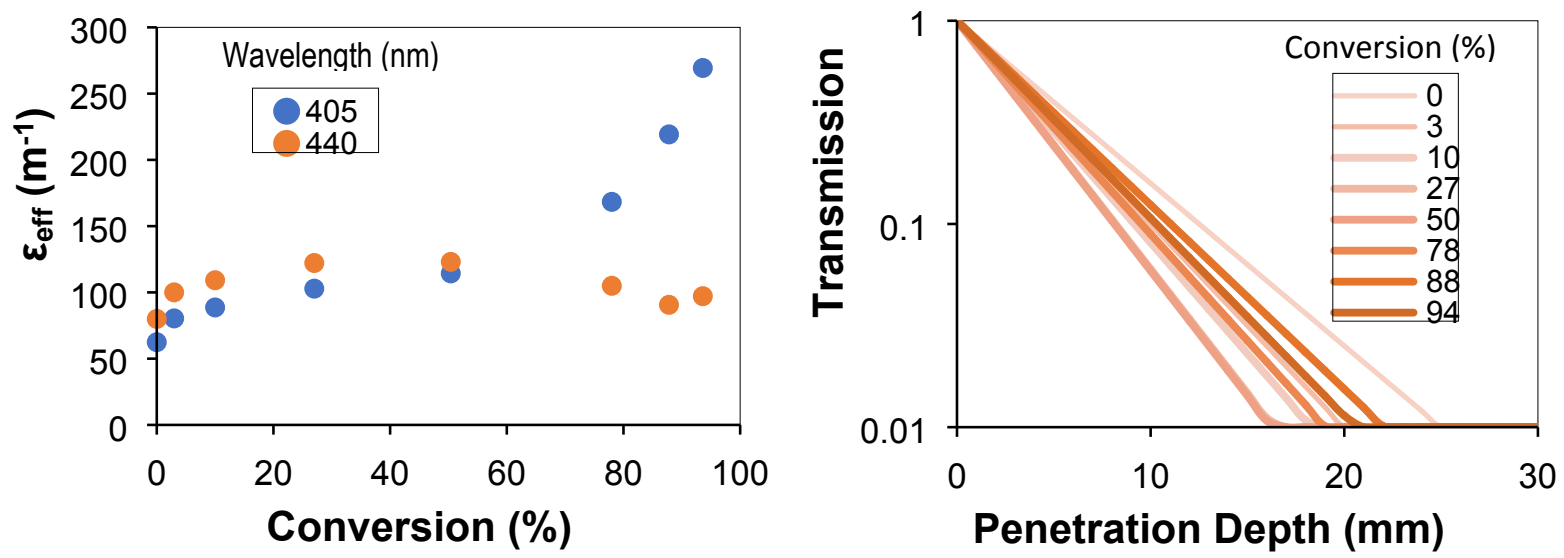

Figure S6: (Left) Effective molar extinction coefficient of the reaction mixture vs. conversion at 405 and 440 nm. (Right) Penetration depth of reaction mixture at $440 \mathrm{~nm}$ vs. conversion. Effective molar extinction coefficient is defined as: $A=\varepsilon_{e f f} l=\sum_{i} \varepsilon_{i} C_{i} l$ 
4. Calculation of Emitted Photon Equivalence

$$
\begin{gathered}
\eta_{e q}=\frac{t n_{p}}{C V}=\frac{t P \lambda}{C V h c N_{A}} \\
\eta_{e q}=\text { photon equivalents } \\
t=\text { time }[\mathrm{s}] \\
n_{p}=\text { molar flow rate of emitted photons }\left[\mathrm{mol} \mathrm{s}^{-1}\right] \\
C=\text { concentration of bromo }-4-\text { trifluorotoluene }\left[\mathrm{mol} \mathrm{L}^{-1}\right] \\
V=\text { volume of reactor }[L] \\
P=\text { lamp output power }[\mathrm{W}] \\
\lambda=\text { emitted photon wavelength }[\mathrm{m}] \\
h=\text { Planck'sconstant }[\mathrm{Js}] \\
c=\text { speed of light }\left[\mathrm{m} \mathrm{s}{ }^{-1}\right] \\
N_{A}=\text { Avogadro's number }
\end{gathered}
$$




\section{General Information}

Unless otherwise specified, flow reactors and fluidic connections were made with commonly available parts from IDEX Health \& Science Technologies, Vapourtec Ltd. (Cambridge UK), or Upchurch Scientific. The tubing used in the construction of these systems included fluoropolymer tubing, either PFA (perfluoroalkoxy alkane) or FEP (fluorinated ethylene propylene), with both types having an outer diameter of $1 / 16$ " and an inner diameter of either 0.030 " or 0.040 " or outer diameters of $1 / 8$ " or $3 / 16$ " with inner diameters of 0.063 " and 0.125 ", respectively. Fluidic connections were made with flat-bottomed (1/4-28) ports and nuts unless noted otherwise. Solutions were transferred using a Masterflex L/S series peristaltic pump unit. Solvents and reagents were purchased from Fisher Scientific and Sigma-Aldrich and were used as received. Starting materials were purchased from Oakwood and Acros and were used as received. Reactions were monitored by quantitative ${ }^{1} \mathrm{H}$ NMR in chloroform-d, using 1,3benzodioxole as the external standard. All ${ }^{1} \mathrm{H}$ NMR spectra were measured on either a $400 \mathrm{MHz}$ or $500 \mathrm{MHz}$ Bruker NMR instrument, with a $20-30 \mu \mathrm{L}$ sample of the reaction solution being diluted with chloroform-d. One equivalent of 1,3-benzodioxole was added to each sample as an external standard.

\section{Photoreactor Design}

For the multikilogram scale experiments, a 100-L chemglass vessel was connected through the bottom valve of the vessel to $1 / 4$ " PTFE tubing via swagelock. The flow rate was controlled by a peristaltic pump and the PTFE tubing was connected to the FEP tubing (3/8" O.D.; 5/16" I.D., Altaflo part no. 200-0375-030-0C, $18 \mathrm{~m}$, total volume of $890 \mathrm{~mL}$ ) which comprised the photoreactor. A 12"x12" square of framing extrusion (part no. 1515-LITE-72) with 3-D printed supports was used to wrap the tubing to the bend radius of the tubing. The tubing was submerged into a recirculating water bath. The temperature of the water bath was controlled by recirculation of the fluid through a $330 \mathrm{~mL}$ stainless steel heat exchanger submerged in an ice water bath. The photoreactor was flanked on either side by panels consisting of fifteen 440-450 nm 100W LED chips (Chanzon, 1DGL-JC-100W-440) for a total of $3 \mathrm{~kW}$ of input power. The panels were cooled by flowing water through a copper pipe embedded into the panel. Thermocouples were installed on the reaction stream before and after the irradiation zone, on the water bath, on the light panels, and on the inlet and outlet of the cooling water on the LED panels. The reaction was allowed to reach steady sate (after three reactor volumes) before collection was begun into a carboy. 


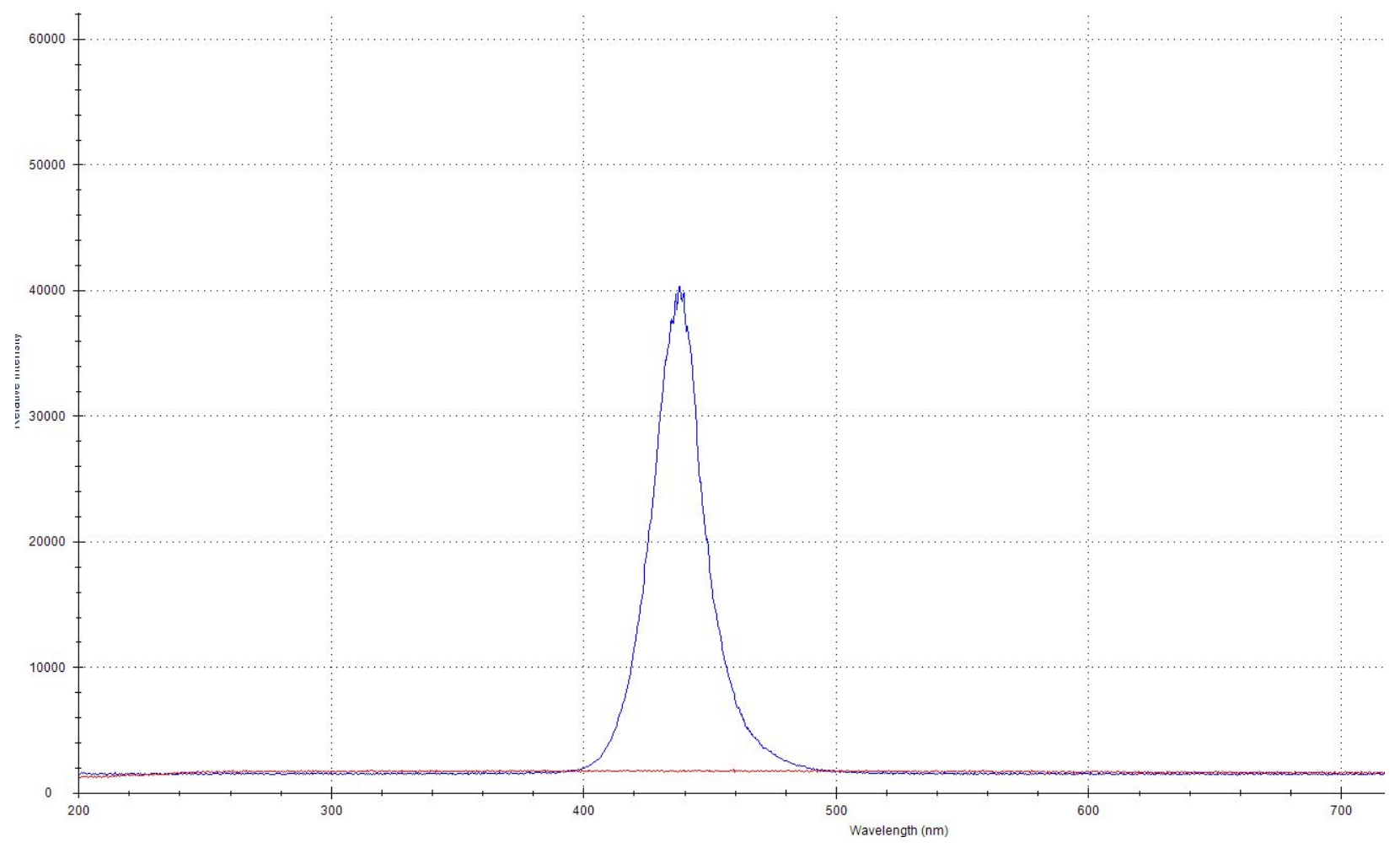

Figure S7: Emission spectrum of LED chips measured with Exemplar LS from BWTEK

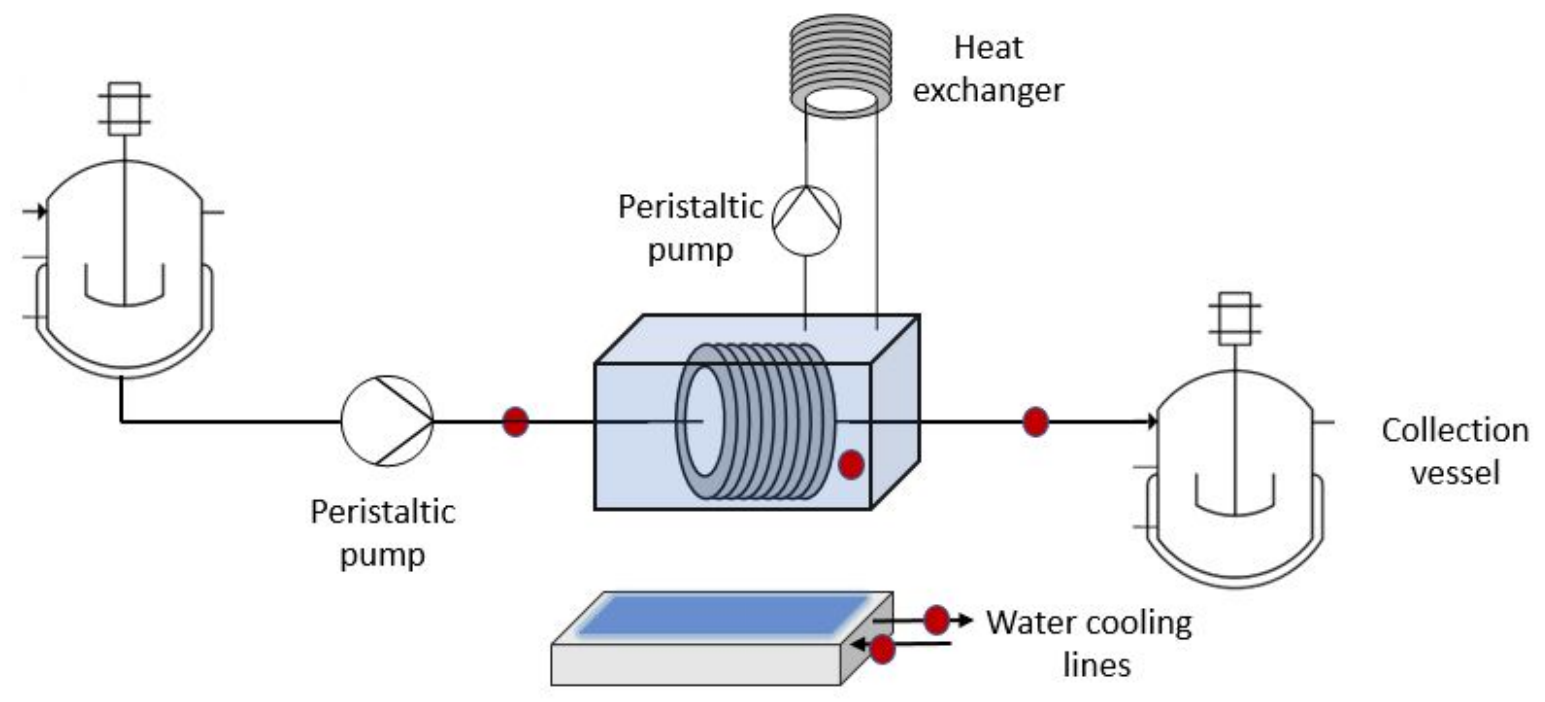

LED light panel

Thermocouples (5)

Figure S8: Process Flow Diagram. For clarity, only one of the two LED light panels is represented 
7. LED Plate Output Power Density, Energy Efficiency, and Stability

$$
\begin{gathered}
\dot{m} C_{p}\left(T_{\text {out }}-T_{\text {in }}\right)=\dot{S}_{L E D} \\
\eta=1-\dot{S}_{L E D} / \dot{E}_{\text {in }} \\
\dot{F}_{L E D}=\frac{\eta \dot{E}_{\text {in }}}{A} \\
\dot{m}=\text { mass flow rate of cooling water }\left[\frac{\mathrm{kg}}{\mathrm{min}}\right]=2.25 \\
C_{p}=\text { water heat capacity }\left[4.184 \frac{\mathrm{J}}{\mathrm{gK}}\right] \\
T_{i}=\text { temperature of cooling water (inlet or outlet) }\left[{ }^{\circ} \mathrm{C}\right] \\
\dot{S}_{L E D}=\text { Energy (Heat)removed from LED plate }[\mathrm{W}]=1110 \\
\dot{E}_{\text {in }}=\text { Total Energy Input }[\mathrm{W}]=1500 \\
\eta=\text { Photon Output Energy Efficiency }=0.26 \\
\dot{F}_{L E D}=\text { output photon power density }\left[\frac{\mathrm{W}}{\mathrm{cm}^{2}}\right]=5.32
\end{gathered}
$$

A macroscopic, steady-state heat balance was applied around the LED plate assembly to calculate the energy efficiency and power output. The heat removed from the LED plate could be calculated by the difference in outlet and inlet temperature of the cooling water and water flow rate. By extension, the fraction of energy emitted as photons could be calculated.

The temperature profiles of the cooling water (inlet and outlet) and power output of the LED plate are shown in Figure S9. The step change in temperature denoted the LEDs being turned on. Steady-state was reached very quickly once the lights were swiched on. Over 210 min, the average $\Delta \mathrm{T}$ in cooling water was $7.1^{\circ} \mathrm{C}$. With a $2.25 \mathrm{~kg} / \mathrm{min}$ cooling water flow rate, $\dot{S}_{L E D}^{a v g}=1.11 \mathrm{~kW}$ and $\eta=0.26$. The emitted power density was then calculated by dividing the energy output of the LEDs by the area of $5 \times 3$ grid of 15 LED chips on the stainless steel plate. Aveage $\dot{F}_{L E D}$ of the LED plate was $0.93 \mathrm{~W} / \mathrm{cm}^{2}$. This average power output density of the plate was in close agreement with the LED power output of a single LED chip (top corner of the assembly) measured at $6.29 \pm 0.02 \mathrm{~W} / \mathrm{cm}^{2}$. The power density of the plate was expected to be lower that of an individual chip due to non-illuminating space between the LEDs. Lastly, the constant measured power density indicated stabilty of the LEDs over extended time and consistent with $10,000 \mathrm{~h}$ lifetime by manufacturers. 


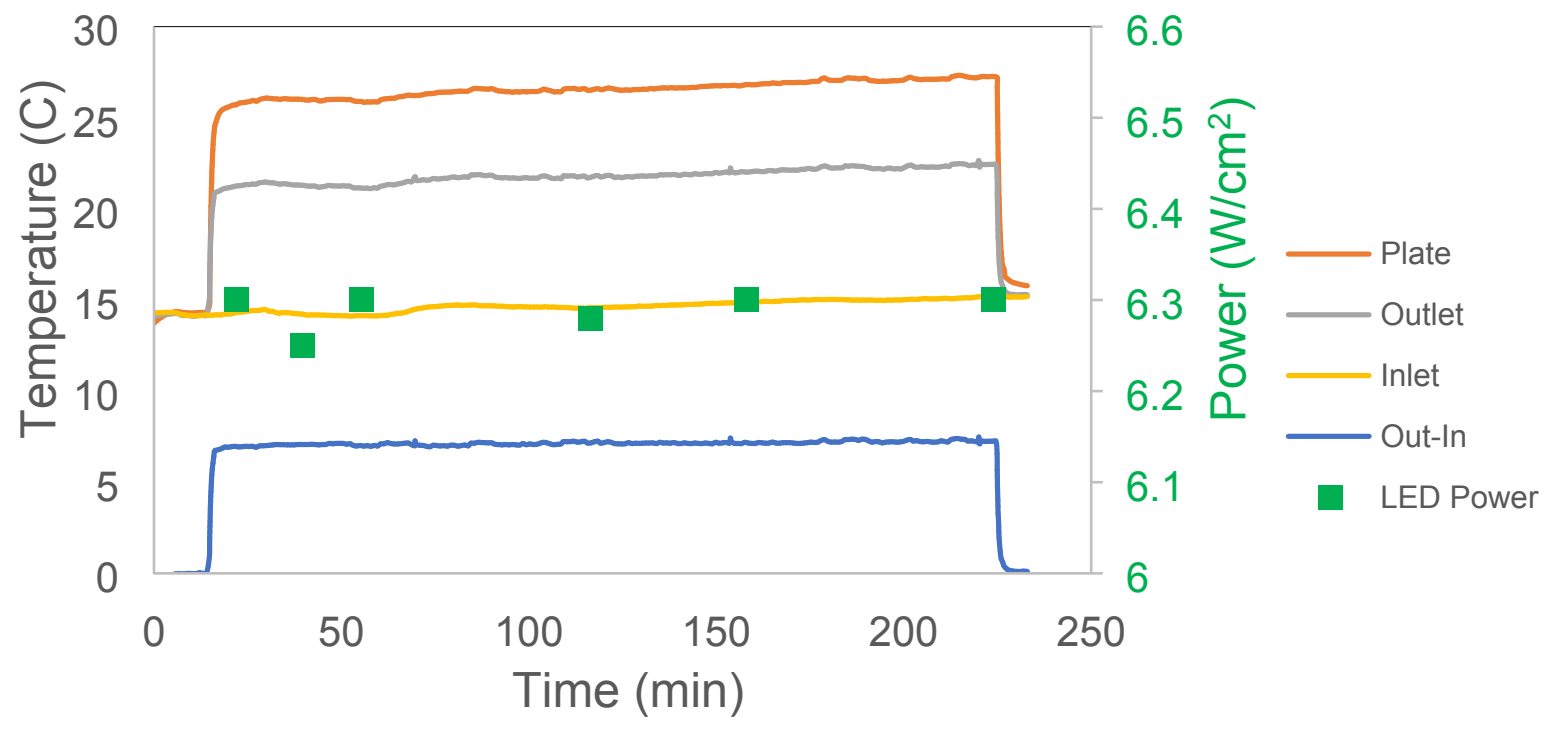

Figure S9: Temperature profiles and output power density at one LED chip in assembly. The power was measured with a PM160 optical power meter (ThorLabs) positioned over an LED chip at the top corner of the LED plate assembly. This position was chosen for consistency in measuring power output stability. 


\section{Design of Experiments (DoE) with Time Profiles}

Reaction Solution Preparation

All reagents were used as received from their respective suppliers.

Initial cross coupling experiments were conducted with a $0.25 \mathrm{M}$ 4-bromobenzotrifluoride with $1.5 \mathrm{eq}(0.375 \mathrm{M})$ pyrrolidine and $1.8 \mathrm{eq}(0.45 \mathrm{M})$ 1,4-diazabicylco[2,2,2] octane solution catalyzed with $7.5 \mathrm{mM}$ (3 mol\%) nickel (II) bromide trihydrate and $0.05 \mathrm{mM}(0.02 \mathrm{~mol} \%)$ tris(2,2'-bipyrimidine)ruthenium (II) hexaflurophosphate. The solution was prepared by adding $\mathrm{NiBr}_{2} \cdot\left(\mathrm{H}_{2} \mathrm{O}\right)_{3}\left(0.20 \mathrm{~g}, 7.4 \times 10^{-4} \mathrm{~mol}, 98 \%\right.$, Sigma-Aldrich) and 1,4-diazabicylco[2,2,2]octane (5.05 g, $0.045 \mathrm{~mol}, \geq 99 \%$, Sigma-Aldrich) to a $100 \mathrm{~mL}$ volumetric flask. Then 4bromobenzotrifluoride $(3.50 \mathrm{~mL}, 5.625 \mathrm{~g}, 0.025 \mathrm{~mol}, 99 \%$, Oakwood Chemical) and pyrrolidine (3.08 mL, $2.67 \mathrm{~g}, 0.038 \mathrm{~mol}$, 99\%, Sigma-Aldrich) were added to the same flask. Dimethylsulfoxide (99\%, Sigma-Aldrich) was added until the solution volume was just under $100 \mathrm{~mL}$, and the flask was sonicated until the $\mathrm{NiBr}_{2} \cdot\left(\mathrm{H}_{2} \mathrm{O}\right)_{3}$ and 1,4-diazabicylco[2,2,2] octane dissolved. Tris(2,2'-bipyrimidine)ruthenium (II) hexaflurophosphate (4.3 mg, 5.0x10-6 mol, $97 \%$, Sigma-Aldrich) was added and the solution volume was filled with DMSO to $100 \mathrm{~mL}$ just before reactions were performed.

\section{Design of Experiments to Identify Optimal Reaction Conditions}

Table S1: Factor and range of values for the design of experiments. Pyrrolidine and DABCO molar equivalents remained constant relative to 4-bromobenzotrifluoride at 1.5 and 1.8 respectively. The optical pathlength was set by the solution volume $1.5,2.25$, and $3 \mathrm{~mL}$ inside scintillation vials. The experimental design space was a two factor, full factorial design ( 24 experiments total).

\begin{tabular}{llll} 
Factor & Low & Center & High \\
\hline 4-bromobenzotrifluoride $(\mathrm{M})$ & 0.25 & 0.325 & 0.4 \\
$\mathrm{Ru}(\mathrm{BPY})_{3}\left(\mathrm{PF}_{6}\right)_{2}(\mathrm{~mol} \%)$ & 0.005 & 0.02 & 0.04 \\
$\mathrm{NiBr}_{2}\left(\mathrm{H}_{2} \mathrm{O}\right)_{3}(\mathrm{~mol} \mathrm{\%})$ & 1 & 3 & 5 \\
Optical length $(\mathrm{cm})$ & 1 & 1.5 & 2
\end{tabular}

The factors and ranges for the design of experiments of the photocatalyzed crosscoupling reaction are listed in Table S1. For a given experiment, $100 \mathrm{~mL}$ of reaction solution was prepared following the same procedure outlined in the Reaction Solution Preparation section for the concentration of each reactant. Reaction solution was transferred into a scintillation vial (17 mm diameter, $60 \mathrm{~mm}$ height, CG-4912-02, ChemGlass) along with a PTFE magnetic stir bar (3.2 mm diameter, $12.7 \mathrm{~mm}$ length, Fisher Scienific). The optical pathlength 
through the reaction solution was varied from 1 to $2 \mathrm{~cm}$ by charging 1.5 to $3 \mathrm{~mL}$ of solution into the vials.

The cross-coupling of 4-bromobenzotrifluoride with pyrrolidine was performed in the Merck photoreactor $^{1}$ equipped with a $5 \mathrm{~W}$ LED light source at $450 \mathrm{~nm}$. The scintillation vials filled with reaction solution were placed inside the center of the holder and submerged into the irradiated zone. The agitation rate was set to $300 \mathrm{rpm}$. The cooling fan was operated at 1500 $\mathrm{rpm}$ for $60 \mathrm{~s}$ before irradiation (pre-cooling), during the reaction, and for $60 \mathrm{~s}$ after the LED was turned off (post-cooling). The duration of irradiation (reaction time) was set to 2, 4, 7, 10, 15, $30,60,120,240,360,450,600,900$, or $1200 \mathrm{~s}$. A different vial with fresh reaction solution was used for each reaction time investigated. After irradiation, the reaction solution was covered prevent further reaction from exposure to ambient light. Samples were diluted in 1:1 acetonitrile:water and filtered. An ultra performance liquid chromatograph (UPLC Infinity II, Agilent Technologies) was equipped with an Agilent ZORBAX Eclipse Plus C-18 column (2.1 $\mathrm{mm} \times 50 \mathrm{~mm}, 1.8 \mu \mathrm{m}$ ) was used to quantify conversion. The UPLC method is shown in Table S2.

Table S2: Mobile phase method for UPLC analysis

\begin{tabular}{lll} 
Time (min) & Acetonitrile (\%) & $0.1 \% \mathrm{H}_{3} \mathrm{PO}_{4} / \mathrm{H}_{2} \mathrm{O}(\%)$ \\
\hline 0 & 10 & 90 \\
\hline 1 & 10 & 90 \\
\hline 7 & 95 & 5 \\
\hline 10 & 95 & 5
\end{tabular}

Conversion of the reaction was quantified as follows:

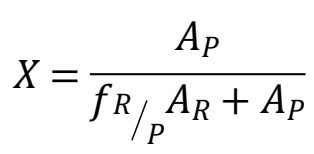

where $\mathrm{X}$ is fractional conversion, $A_{R}$ is the area of 4-bromobenzotrifluoride (reactant), $A_{P}$ is the area of 1-(4-(trifluoromethyl)phenyl)pyrrolidine (product), and $\mathrm{f}_{\mathrm{R} / \mathrm{P}}$ is the relative response factor of the reactant to the product at $210 \mathrm{~nm}$. From conversion profiles, the time profiles of aniline product formation were calculated

Design of Experiments with Time Profiles

An important aspect of scaling photochemcial reactions was to develop an approach to quickly ascertain the optimal reaction conditions to 1) maximize productivity of the desired product and 2) maximize conversion to ease downstream separation/purification. This optimization should result from understanding the effect of process parameters and their interactions on conversion and reaction rate. Due to the complex underlying mechanism for this dual catalytic metallophotoredox reaction system, our optimization approach was to employ data-driven methodologies such as design of experiments (DoE) with time profiles. Experiments were performed in a two level, full factorial design with the 4-bromobenzotrifluoride (M), 
$\mathrm{Ru}(\mathrm{BPY})_{3}\left(\mathrm{PF}_{6}\right)_{2}(\mathrm{~mol} \%), \mathrm{NiBr}_{2}\left(\mathrm{H}_{2} \mathrm{O}\right)_{3}(\mathrm{~mol} \%)$, and optical pathlength $(\mathrm{cm})$ as factors. The bounds for this DoE were chosen to explore the operating space of higher productivity while potentially minimizing catalyst loadings and tube diameter of the plug flow reactor.

Examining the experimental data (Figure S10, Figure S11, and Table S3), the most significant factors for conversion and productivity were 1) $\mathrm{NiBr}_{2}$ loading, 2) optical pathlength, 3) concentration of 4-bromobenzotrifluoride, 4) the interactions between $\mathrm{NiBr}_{2}$ and the pathlength and 4-bromobenzenetrifluoride. From these time profiles, $\mathrm{NiBr}_{2}$ loading enhanced end conversion and productivity (Figure S12). This was due to the initial rate enhancement from higher $\mathrm{NiBr}_{2}$ loading by drastically shortening an initial induction period. Two other factors, 4bromobenzenetrifluoride concentration and pathlength, had opposite contributions to conversion and productivity. Unsurprisingly, productivity increased with higher 4-bromobenzenetrifluoride concentration and longer pathlength (higher reaction volume) (Figure S12, Figure S13). However, higher 4-bromobenzenetrifluoride concentration elongated the induction period (Figure $\mathrm{S} 13$, low $\mathrm{NiBr}_{2}$ loading), supporting its adverse effect to conversion and productivity. The pathlength also delayed the onset of reaction at low $\mathrm{NiBr}_{2}$ loading (Figure S14) as conversion increased more rapidly for short reaction times at $1 \mathrm{~cm}$ pathlength. However, the interaction of $\mathrm{NiBr}_{2}$ with the pathlength eliminated the induction period and any difference in the intial reaction rate. This was evident in Figure S14 when comparing time series of different pathlengths and $\mathrm{NiBr}_{2}$ loadings at high 4-bromobenzenetrifluoride concentration. This valuable insight indicated that the deleterious effects of long pathlengths could be mitigated if the $\mathrm{NiBr}_{2}$ concentration was sufficiently high in the reaction mixture. Lastly, the photocatalyst impact on conversion and productivity was mixed. The photocatalyst loading appeared insignificant for productivity, conversion, and reaction rate at high $\mathrm{NiBr}_{2}$ loading (Figure S13). It also had slightly negative and positive effects on the initial rate and end conversion respectively at low $\mathrm{NiBr}_{2}$ loading. Photocatalyst concentration (interaction with 4-bromobenzenetrifluoride) was insignificant. The interaction of $\mathrm{Ru}(\mathrm{BPY})_{3}$ with $\mathrm{NiBr}_{2}$ (Figure S15) had some positive impact on the initial reaction rate and end conversion and productivity. The positive interaction between $\mathrm{Ru}$ and $\mathrm{Ni}$ catalysts were likely because of the interaction between the photo and thermal catalytic cycles. Despite these positive effects, the effects of $\mathrm{Ru}(\mathrm{BPY})_{3}$ and $\mathrm{NiBr}_{2}$ loading on the initial reaction rate (Figure S15) show that higher $\mathrm{Ru}(\mathrm{BPY})_{3}$ loading had a smaller impact compared to higher $\mathrm{NiBr}_{2}$ loading.

From these insights conveyed by the DoE, the conversion and productivity of the reaction were improved by maximizing 1) 4-bromobenzenetrifluoride concentration, 2) $\mathrm{NiBr}_{2}$ loading, and 3) pathlength. Within the DoE design space, the concentration of 4-bromobenzenetrifluoride was set to $0.4 \mathrm{M}$ to maximize productivity. While doing this would slow the initial conversion rate, increasing $\mathrm{NiBr}_{2}$ loading to $5 \mathrm{~mol} \%$ offset this loss by increasing the initial reaction rate and improving both conversion and productivity. Higher $\mathrm{NiBr}_{2}$ loadings were explored $(>0.02 \mathrm{M})$, but the solubility of $\mathrm{NiBr}_{2}$ in $\mathrm{DMSO}$ caused this regime to be impractical. The optical pathlength was also increased to $2 \mathrm{~cm}$ to increase productivity. The positive interaction of $\mathrm{NiBr}_{2}$ with pathlengths counteracted the induction period such that lengthening the pathlength would not slow the initial reaction rate. Lastly, the $\mathrm{Ru}(\mathrm{BPY})_{3}\left(\mathrm{PF}_{6}\right)_{2}$ concentrations was selected such that $>99 \%$ absorbance (full absorption) would be achieved across the pathlength. This was 
achieved at $0.04 \mathrm{~mol} \% \mathrm{Ru}(\mathrm{BPY})_{3}\left(\mathrm{PF}_{6}\right)_{2}$, but there were minimal differences in the initial reaction rate and end productivity at $0.005 \mathrm{~mol} \% \mathrm{Ru}(\mathrm{BPY})_{3}\left(\mathrm{PF}_{6}\right)_{2}$. Under these optimized reaction conditions, the end conversion increased by $14 \%$ from $85 \%$ to $99 \%$ and productivity increased 2.5-fold (Figure S16). The high $\mathrm{NiBr}_{2}$ loading eliminated the induction period and accelerated the initial conversion rate despite a higher 4-bromobenzenetrifluoride concentration and longer optical pathlength. Likewise, the productivity was significantly increased by increasing the 4-bromobenzenetrifluoride concentration and the optical pathlength.

The same principles were applied to optimize the reaction conditions in the plug flow reactor. Table $\mathrm{S} 3$ shows the optimized reaction conditions guided by the DoE. As within the DoE design space, the 4-bromobenzenetrifluoride and $\mathrm{NiBr}_{2}$ concentrations were set to $0.4 \mathrm{M}$ and $5 \mathrm{~mol} \%$ respectively. The pathlength was maximized set to the $7.9 \mathrm{~mm}$, the largest inner diameter tubing tested in the plug flow reactor (Table 1, main text). Lastly, the concentration of $\mathrm{Ru}(\mathrm{BPY})_{3}\left(\mathrm{PF}_{6}\right)_{2}$ was set to $0.02 \mathrm{~mol} \%$. These reaction conditions were then used in both laboratory and scale-up experiments. 

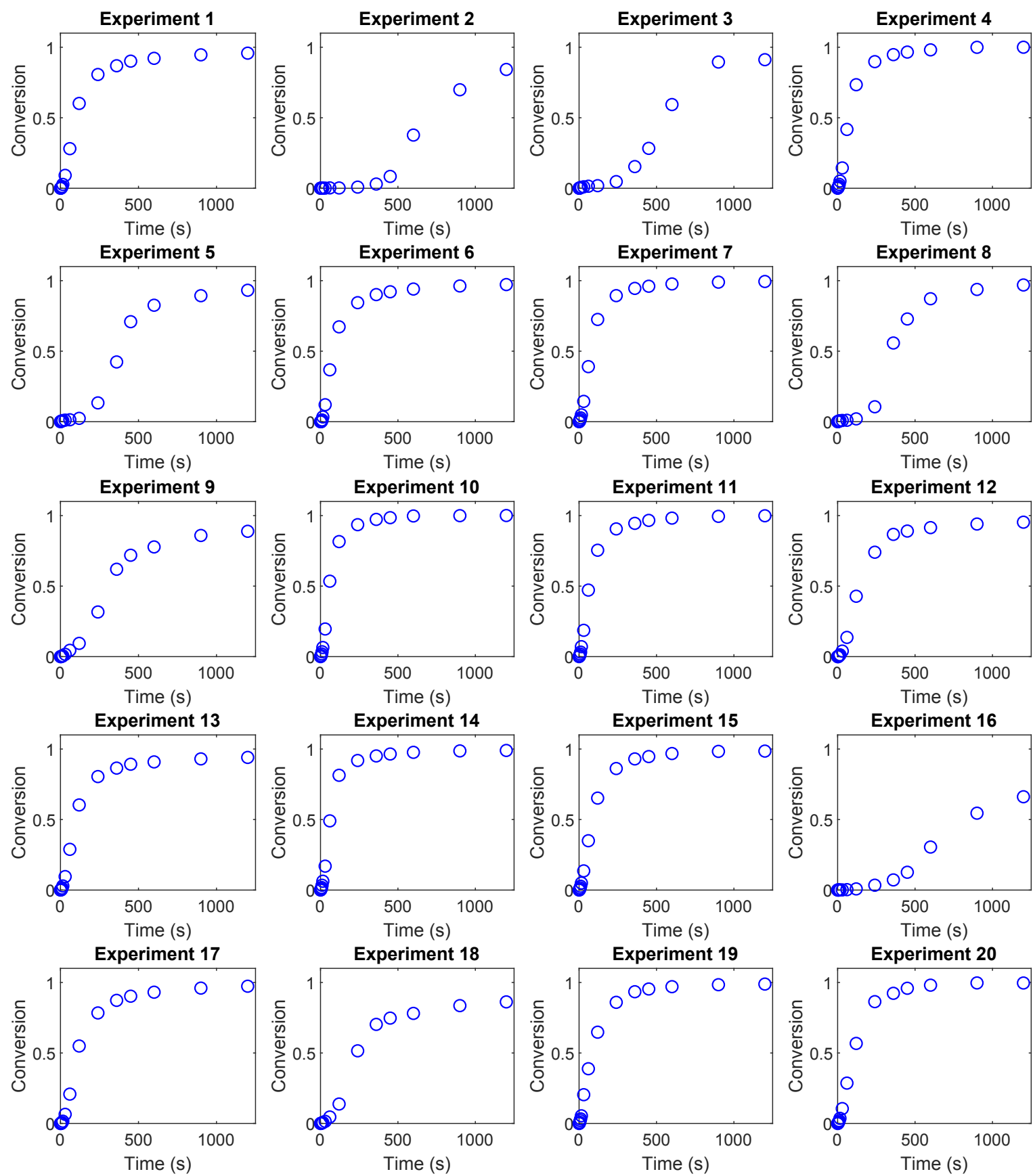

Figure S10: DoE experimental conversion of the photocatalyzed cross-coupling amination. 

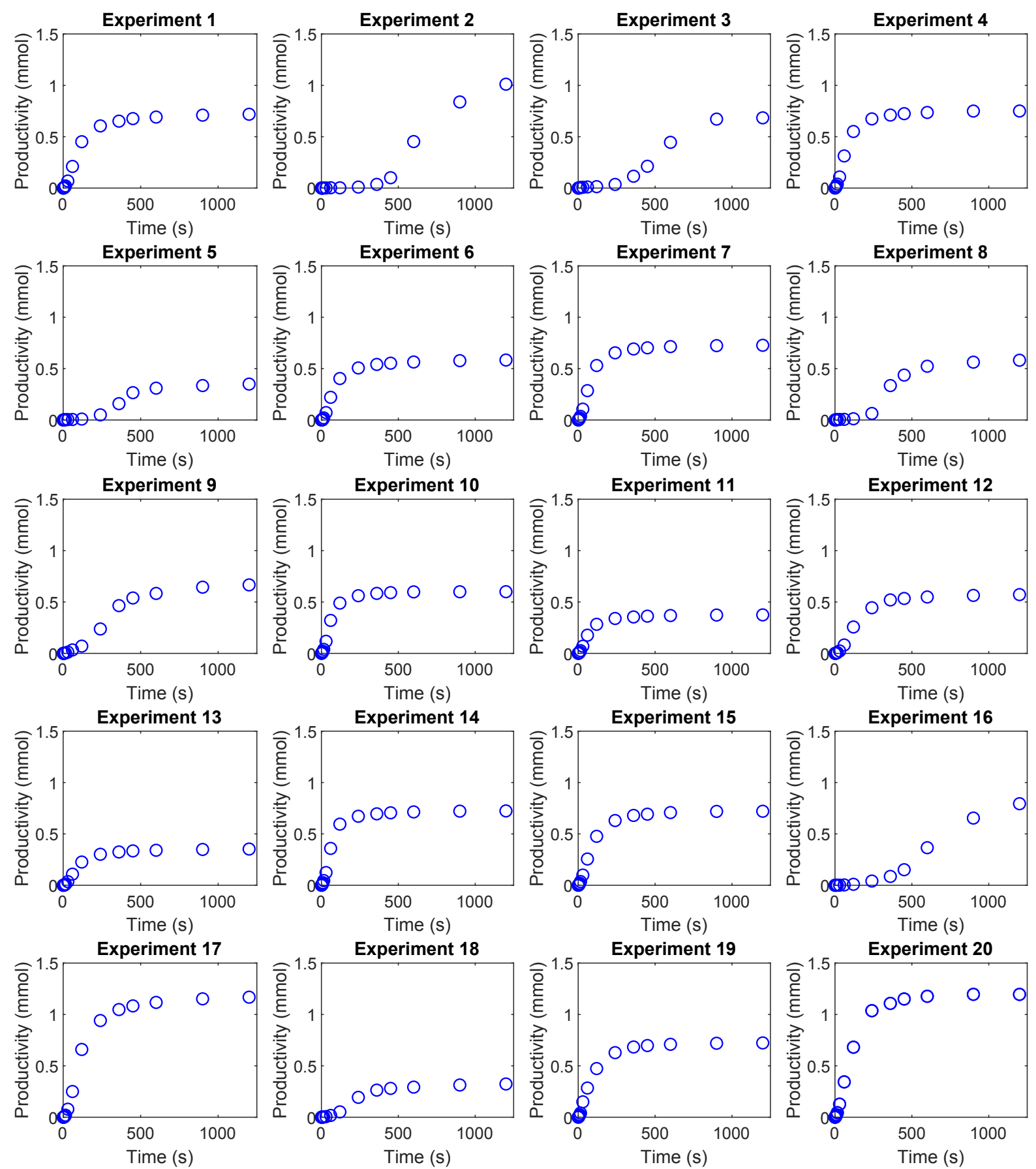

Figure S11: DoE experimental productivity of the photocatalyzed cross-coupling amination. 
Table S3: Factor levels of each experiment in DoE. -1 denotes low value, 0 is centerpoint, +1 is high value.

\begin{tabular}{ccccc} 
Experiment & 4-bromobenzenetrifluoride & $\mathrm{Ru}(\mathrm{BPY})_{3}\left(\mathrm{PF}_{6}\right)_{2}$ & $\mathrm{NiBr}_{2}\left(\mathrm{H}_{2} \mathrm{O}\right)_{3}$ & Optical Pathlength \\
\hline 1 & -1 & -1 & -1 & 1 \\
\hline 2 & 1 & 1 & -1 & 1 \\
\hline 3 & -1 & 1 & 1 & -1 \\
\hline 4 & -1 & 1 & -1 & -1 \\
\hline 5 & -1 & 1 & 1 & 0 \\
\hline 6 & 1 & -1 & 0 & -1 \\
\hline 7 & 0 & 0 & -1 & -1 \\
\hline 8 & 1 & 1 & -1 & -1 \\
\hline 9 & -1 & -1 & 1 & -1 \\
\hline 10 & 1 & 1 & 1 & -1 \\
\hline 11 & -1 & 1 & -1 & 0 \\
\hline 12 & 1 & -1 & 1 & 0 \\
\hline 13 & -1 & -1 & 0 & 1 \\
\hline 14 & 0 & 0 & 0 & -1 \\
\hline 15 & 0 & 0 & -1 & -1 \\
\hline 16 & 1 & -1 & -1 & 0 \\
\hline 17 & 1 & -1 & 0 & 1 \\
\hline 18 & -1 & -1 & 1 & 1 \\
\hline 19 & 0 & 0 & 1 & 1 \\
\hline 20 & 1 & 1 & 1 & 1 \\
\hline
\end{tabular}



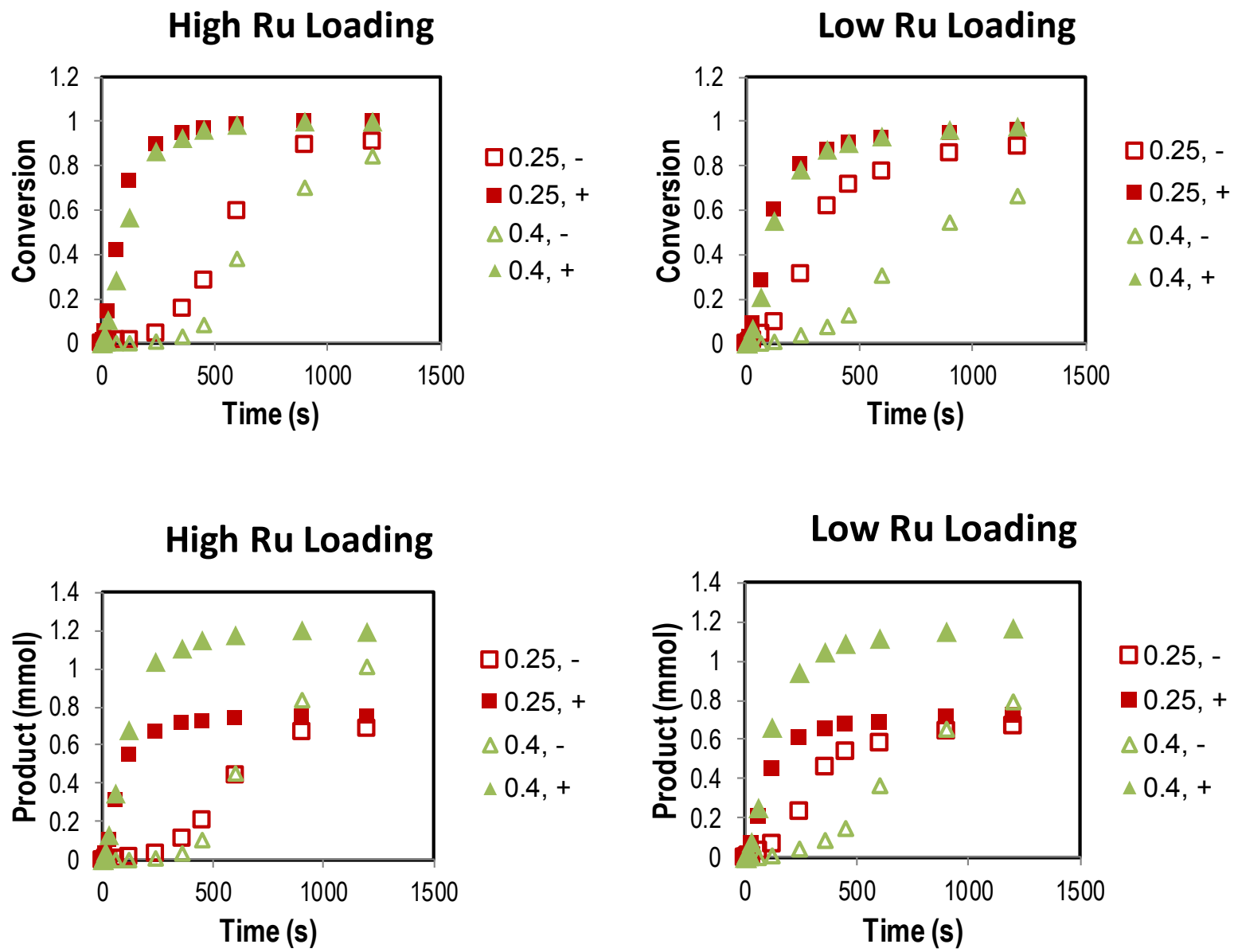

Figure S12: Conversion and aniline product production (mmol) for low (-, open symbols) and high (+, closed symbols) $\mathrm{NiBr}_{2}$ loading at $0.25 \mathrm{M}(\square)$ or $0.4 \mathrm{M}(\triangle)$ 4-bromobenzenetrifluoride. (Left column) $0.04 \mathrm{~mol} \%$ $\mathrm{Ru}(\mathrm{BPY})_{3}(\mathrm{PF})_{2}$ and $2 \mathrm{~cm}$ pathlength. (Right column) $0.005 \mathrm{~mol} \% \mathrm{Ru}(\mathrm{BPY})_{3}(\mathrm{PF} 6)_{2}$ and $2 \mathrm{~cm}$ pathlength. Reactions performed in a Merck photoreactor at $\lambda=450 \mathrm{~nm}$. 

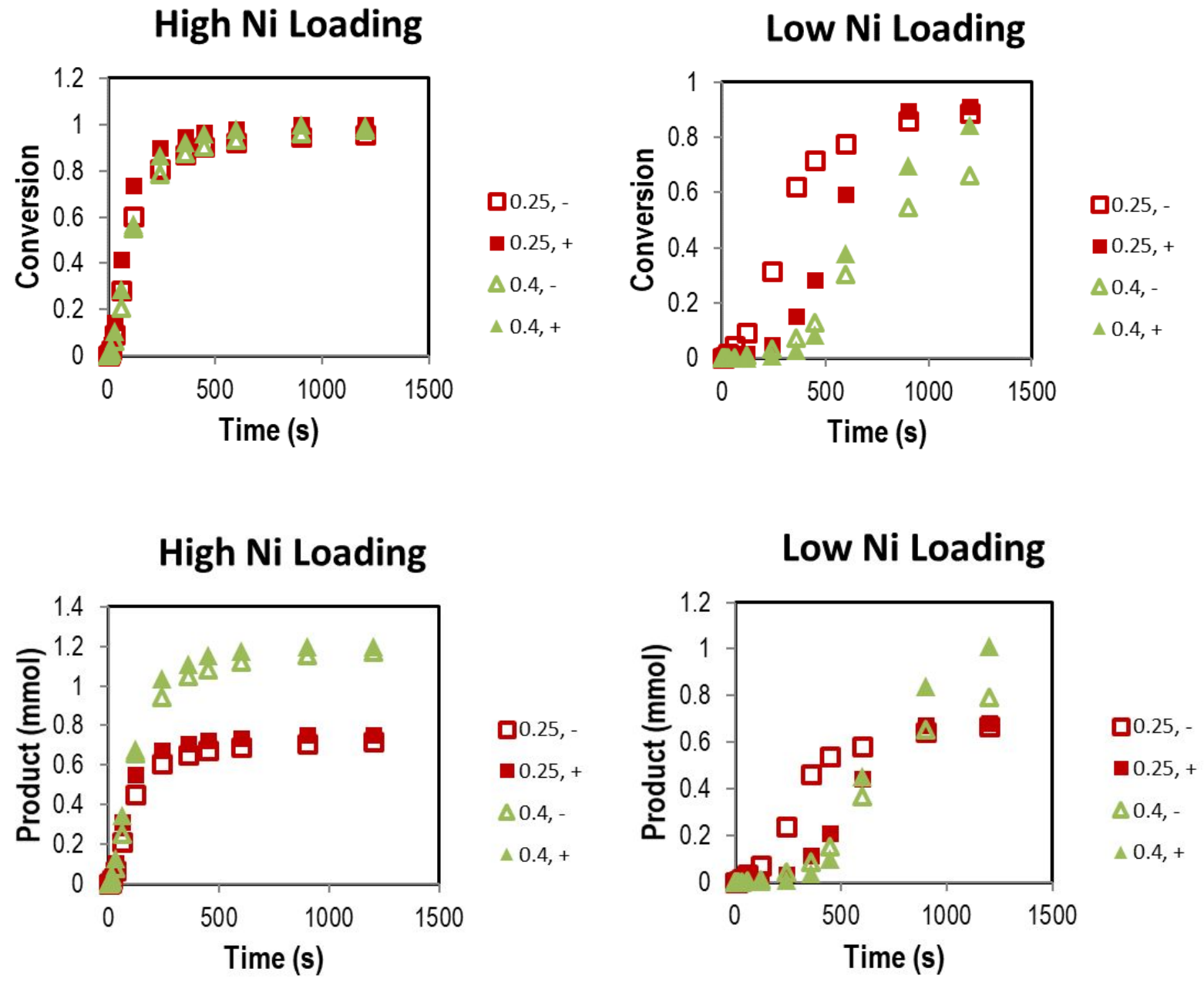

Figure S13: Conversion and aniline product production (mmol) for low (-, open symbols) and high ( + , closed symbols) Ru(BPY), loading at $0.25 \mathrm{M}(\boldsymbol{n})$ or $0.4 \mathrm{M}(\Delta)$ 4-bromobenzenetrifluoride. (Left column) $5 \mathrm{~mol} \%$ $\mathrm{NiBr}_{2}$ and $2 \mathrm{~cm}$ pathlength. (Right column) $1 \mathrm{~mol} \% \mathrm{NiBr}_{2}$ and $2 \mathrm{~cm}$ pathlength. Reactions performed in a Merck photoreactor at $\lambda=450 \mathrm{~nm}$. 

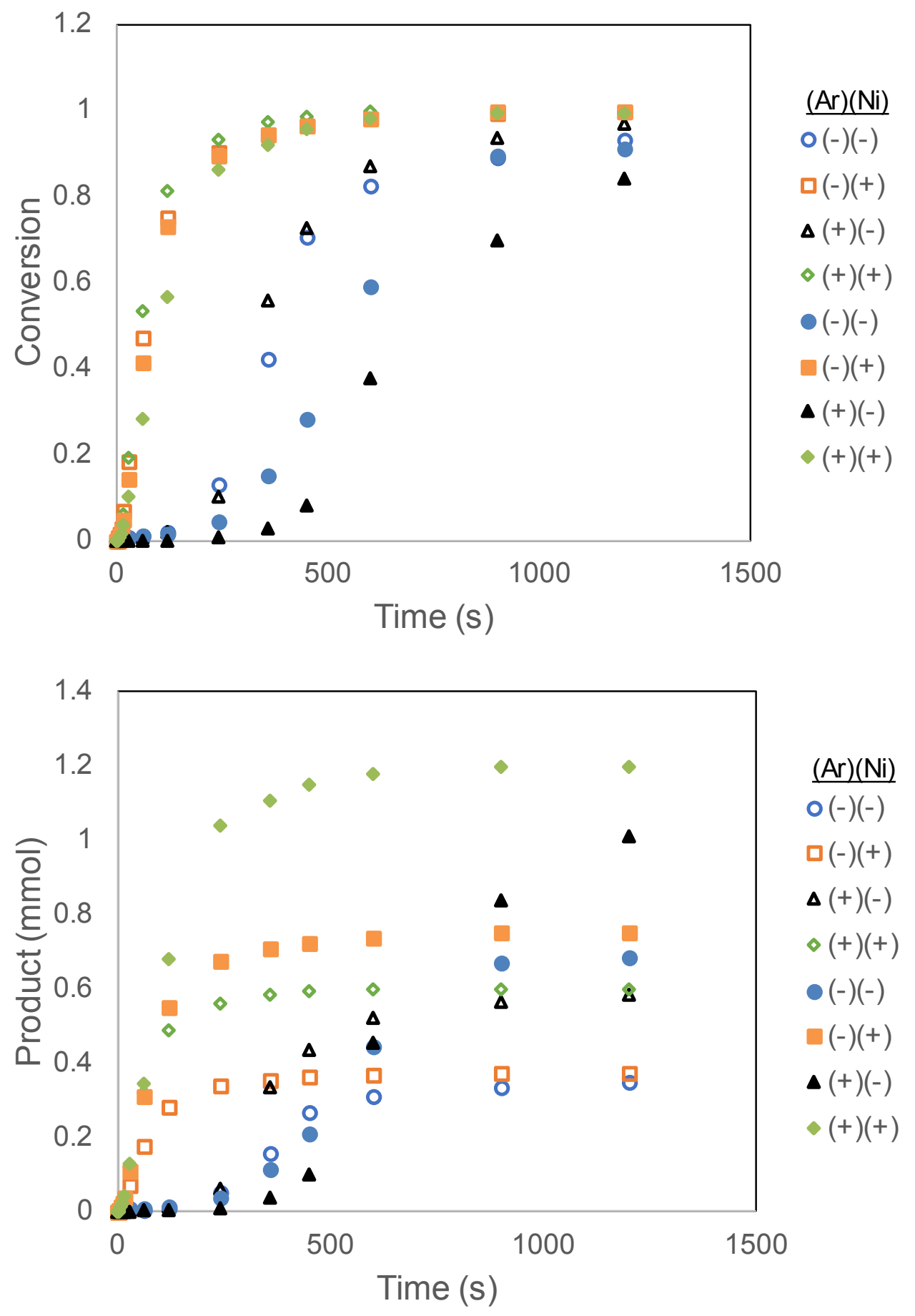

Figure S14: Conversion and aniline production (mmol) for $1 \mathrm{~cm}$ (open symbols) and $2 \mathrm{~cm}$ (closed symbols) pathlength and $0.04 \mathrm{~mol} \% \mathrm{Ru}(\mathrm{BPY})_{3}(\mathrm{PF} 6)_{2}$. $(-)$ and $(+)$ denote low and high factor values for 4bromobenzenetrifluoride and $\mathrm{NiBr}_{2}$ loading respectively. Reactions performed in a Merck photoreactor at $\lambda$ $=450 \mathrm{~nm}$. 

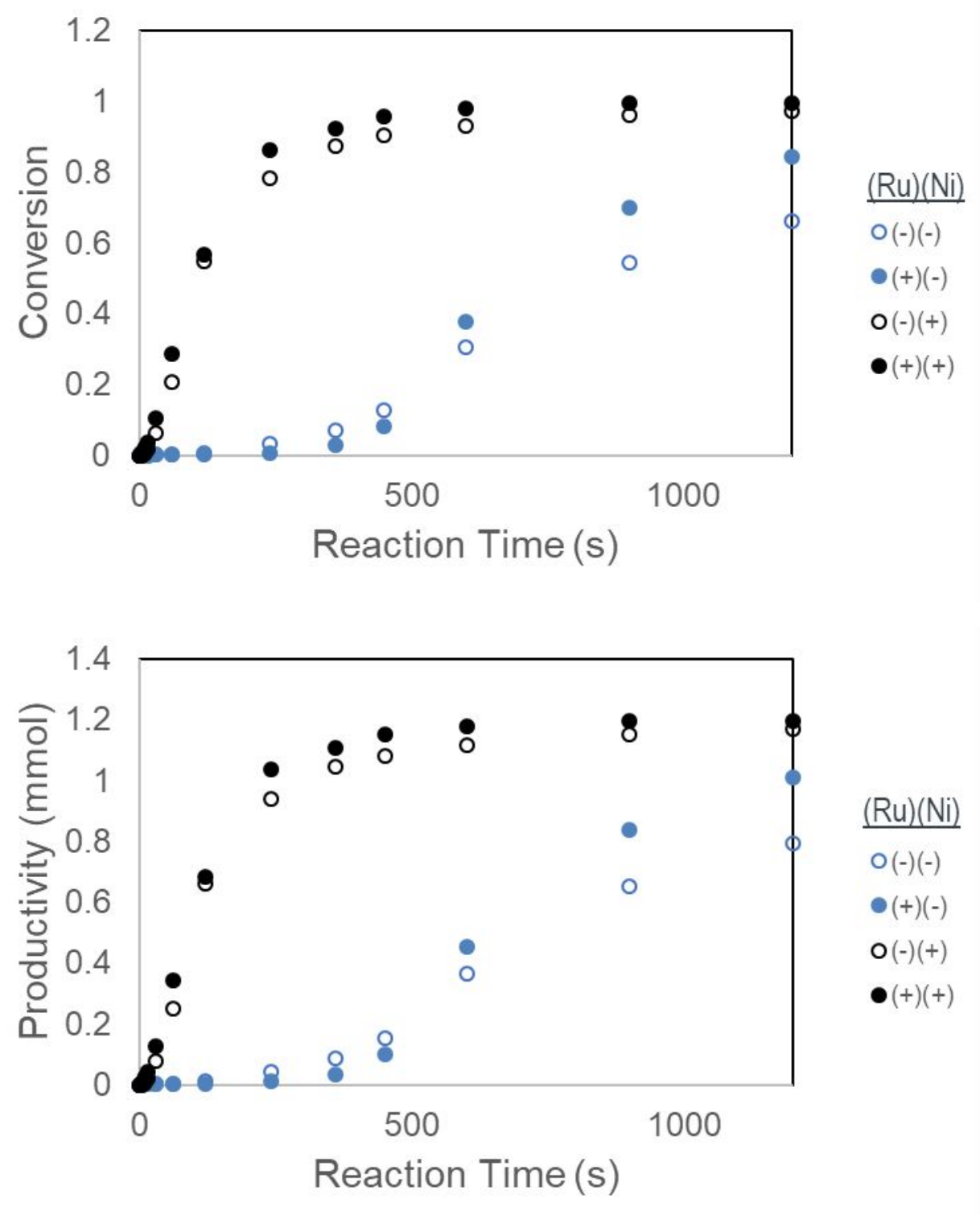

Figure S15: Conversion and aniline product production (mmol) for low (blue symbols) and high (black symbols) Ru(BPY) $)_{3}$ loading at 0.005 mol\% (open symbols) or 0.04 mol\% (closed symbols) Ru(BPY) $)_{3} .0 .40 \mathrm{M}$ 4-bromobenzenetrifluoride and $2 \mathrm{~cm}$ pathlength. Reactions performed in a Merck photoreactor at $\lambda=450$ nm. 


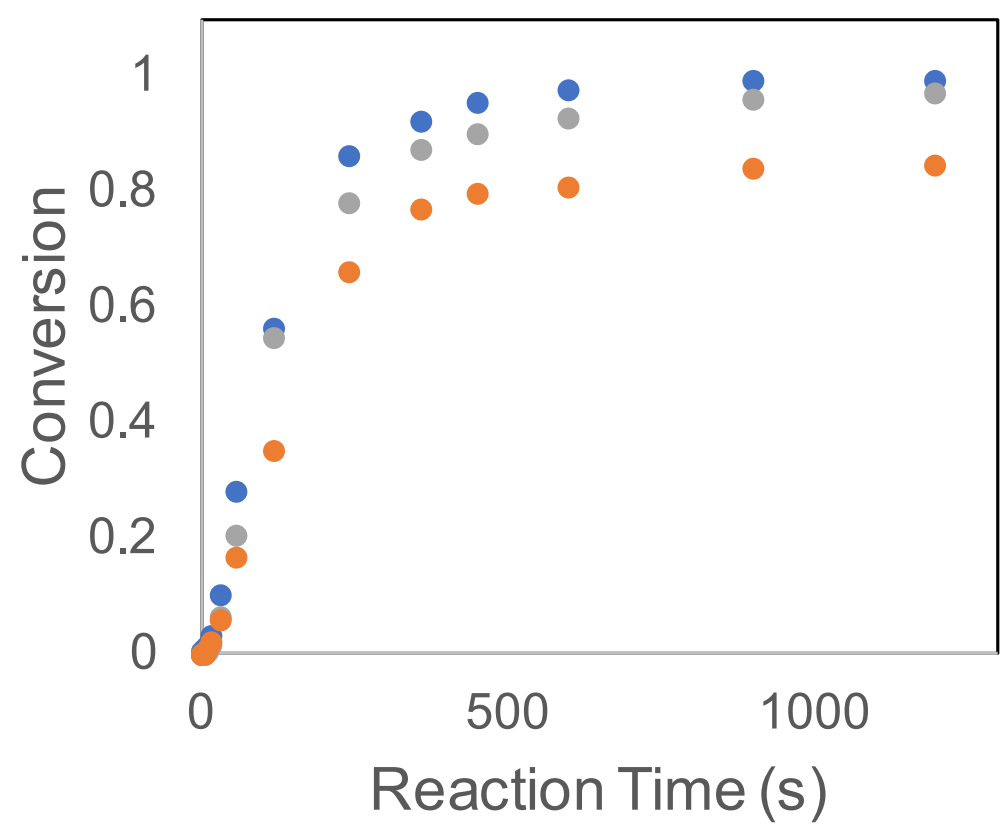

- Optimized

- Opt, $0.005 \mathrm{~mol} \% \mathrm{Ru}$

- Centerpoint

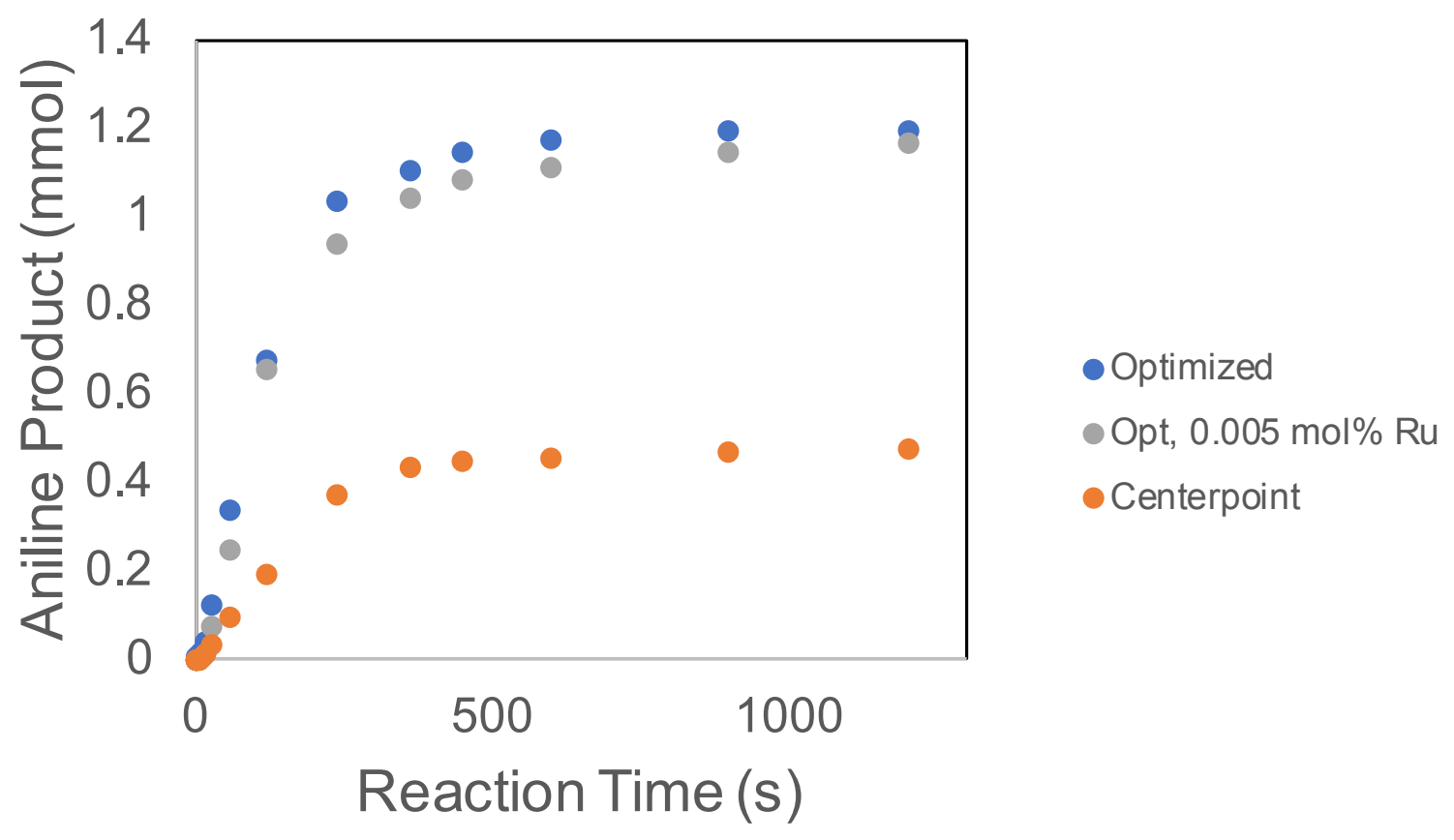

Figure S16: (Top) conversion and (Bottom) Aniline product formation profiles of optimized reaction conditions versus the previous reaction conditions within the design space. Optimized reaction conditions: $0.4 \mathrm{M}$ 4-bromobenzenetrifluoride, $0.04 \mathrm{~mol} \% \mathrm{Ru}(\mathrm{BPY})_{3}\left(\mathrm{PF}_{6}\right)_{2}, 5 \mathrm{~mol} \% \mathrm{NiBr}_{2}\left(\mathrm{H}_{2} \mathrm{O}\right)_{3}, 2 \mathrm{~cm}$ pathlength. Centerpoint conditions: $0.25 \mathrm{M}$ 4-bromobenzenetrifluoride, $0.02 \mathrm{~mol} \% \operatorname{Ru}(\mathrm{BPY})_{3}\left(\mathrm{PF}_{6}\right)_{2}, 3 \mathrm{~mol} \%$ $\mathrm{NiBr}_{2}\left(\mathrm{H}_{2} \mathrm{O}\right)_{3}, 1.5 \mathrm{~cm}$ pathlength. Reactions were performed in a Merck photoreactor with $\lambda=450 \mathrm{~nm}$. 


\section{Procedure for the Multi-Kilogram Scale Flow Reaction}

To a 100 liter vessel were added $16 \mathrm{~L}$ DMSO, 4-Bromobenzotrifluoride $(9.00 \mathrm{~kg}, 40.0 \mathrm{~mol})$, pyrrolidine $(4.27 \mathrm{~kg}, 60.0 \mathrm{~mol})$, nickel (II) bromide trihydrate (545 g, $2.00 \mathrm{~mol})$, and 1,4diazabicyclo[2.2.2] octane $(8.08 \mathrm{~kg}, 72.0 \mathrm{~mol})$. An additional $72 \mathrm{~L}$ of DMSO was used to rinse the transfer equipment into the vessel. The mixture was stirred overnight to completely dissolve the nickel salt. The $\mathrm{Ru}(\mathrm{bpy})_{3}\left(\mathrm{PF}_{6}\right)_{2}(6.88 \mathrm{~g}, 8.00 \mathrm{mmol})$ was added as a solution in $50 \mathrm{~mL}$ of DMSO. The bottom of the vessel was fitted with 1 " tubing connected via swagelock to the $1 \mathrm{~L}$ plug flow reactor (3/16" OD, 1/8" ID). The flow rate was controlled with a peristaltic pump. Thermocouples were installed on the reaction stream prior to and immediately following the photochemical reactor. The reactor was immersed in a recirculating water bath to maintain the desired temperature. The temperature of the bath was recorded with a thermocouple. The LED plates were installed on both sides of the water bath and were cooled by single pass water. The temperature of the incoming and spent water were recorded by thermocouple in addition to the temperature of the aluminum plate. Each LED plate was connected to a power supply providing $1500 \mathrm{~W}(100 \mathrm{~V}, 15 \mathrm{~A})$ of power. The photoreactor was surrounded by an orange polycarbonate shell to minimize light exposure to the scientists. The reactor was allowed to reach steady state and then bulk material was collected. Additional slipstream samples were taken every 15 minutes to measure the stability of the system.

Reaction was monitored by ${ }^{1} \mathrm{H}$ NMR, diluting sample in deuterated chloroform- $\mathrm{d}_{3}$. Conversion was established by comparing the peak of starting material (doublet, $7.49 \mathrm{ppm}$ ) to peak of product $(7.42 \mathrm{ppm})$. Representative ${ }^{1} \mathrm{H}$ NMR are presented in figure S17 and S18. The recorded ${ }^{1} \mathrm{H}$ NMR spectrum for the product is consistent with previous reports in the literature. ${ }^{2}$ 


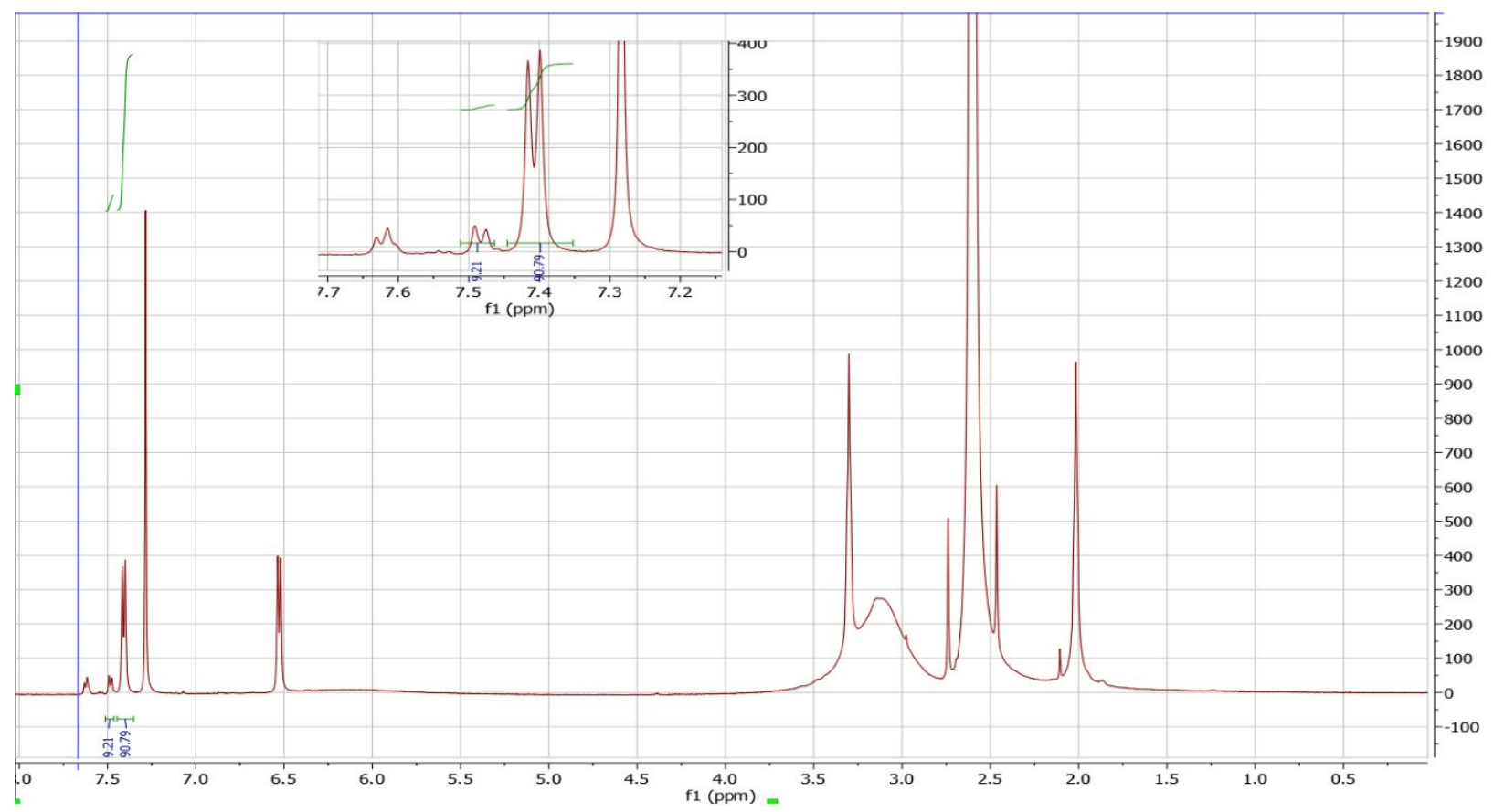

Figure S17: Representative ${ }^{1} \mathrm{H}$ NMR of high conversion experiment.

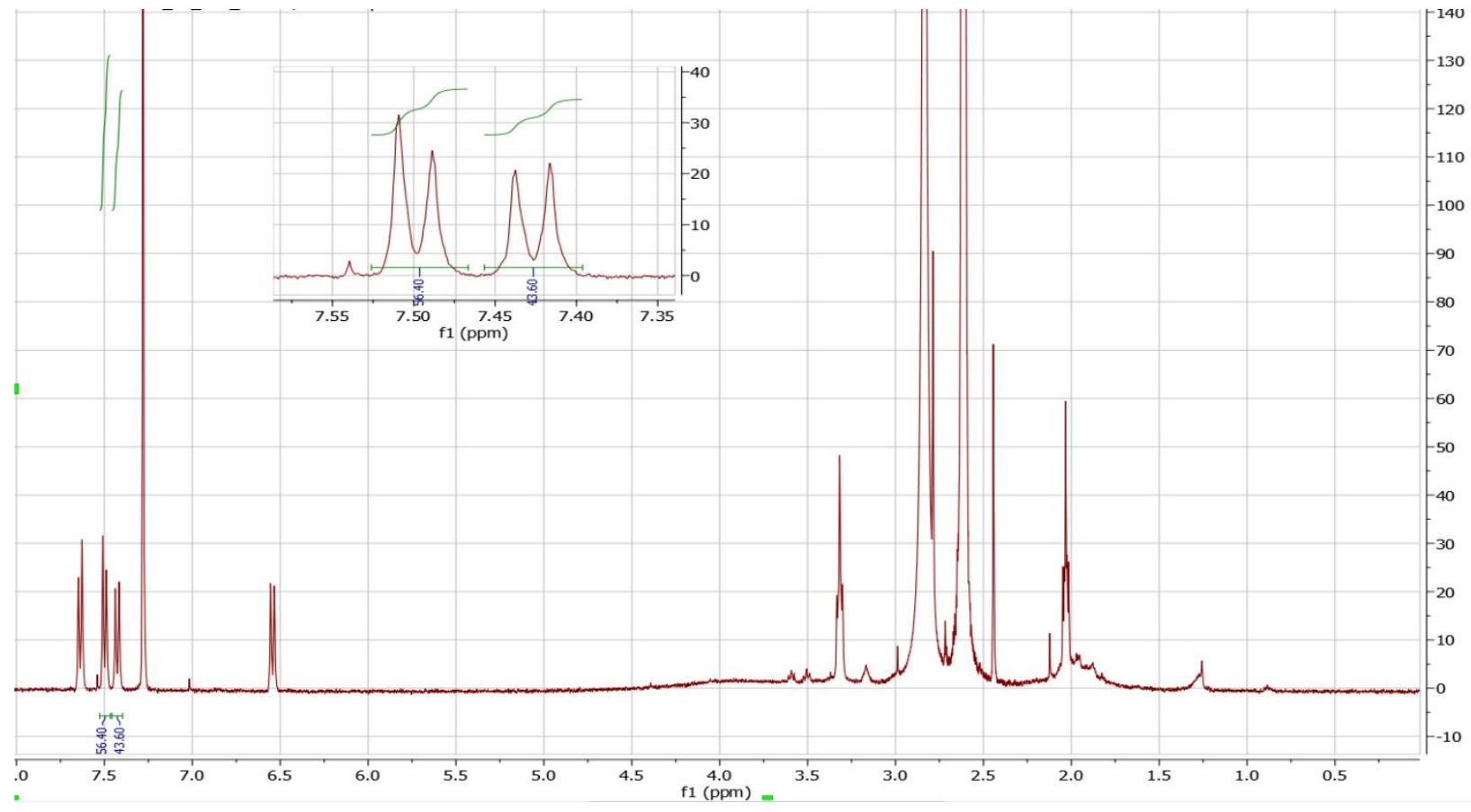

Figure S18: Representative ${ }^{1} \mathrm{H}$ NMR of high productivity experiment.

Assay yield was obtained by adding a known amount of external standard (1,3-Benzodioxole) to $2 \mathrm{~mL}$ of reaction mixture and dissolve $30 \mu \mathrm{L}$ of the resulting mixture in $500 \mu \mathrm{L}$ of deuterated chloroform- $\mathrm{d}_{3}$. ${ }^{1} \mathrm{H}$ NMR for high conversion experiment is presented in figure S19 and the ones 
for high productivity experiment are presented in figure S20 and S21. Assay yield for both experiments are presented in tables S4-S6.

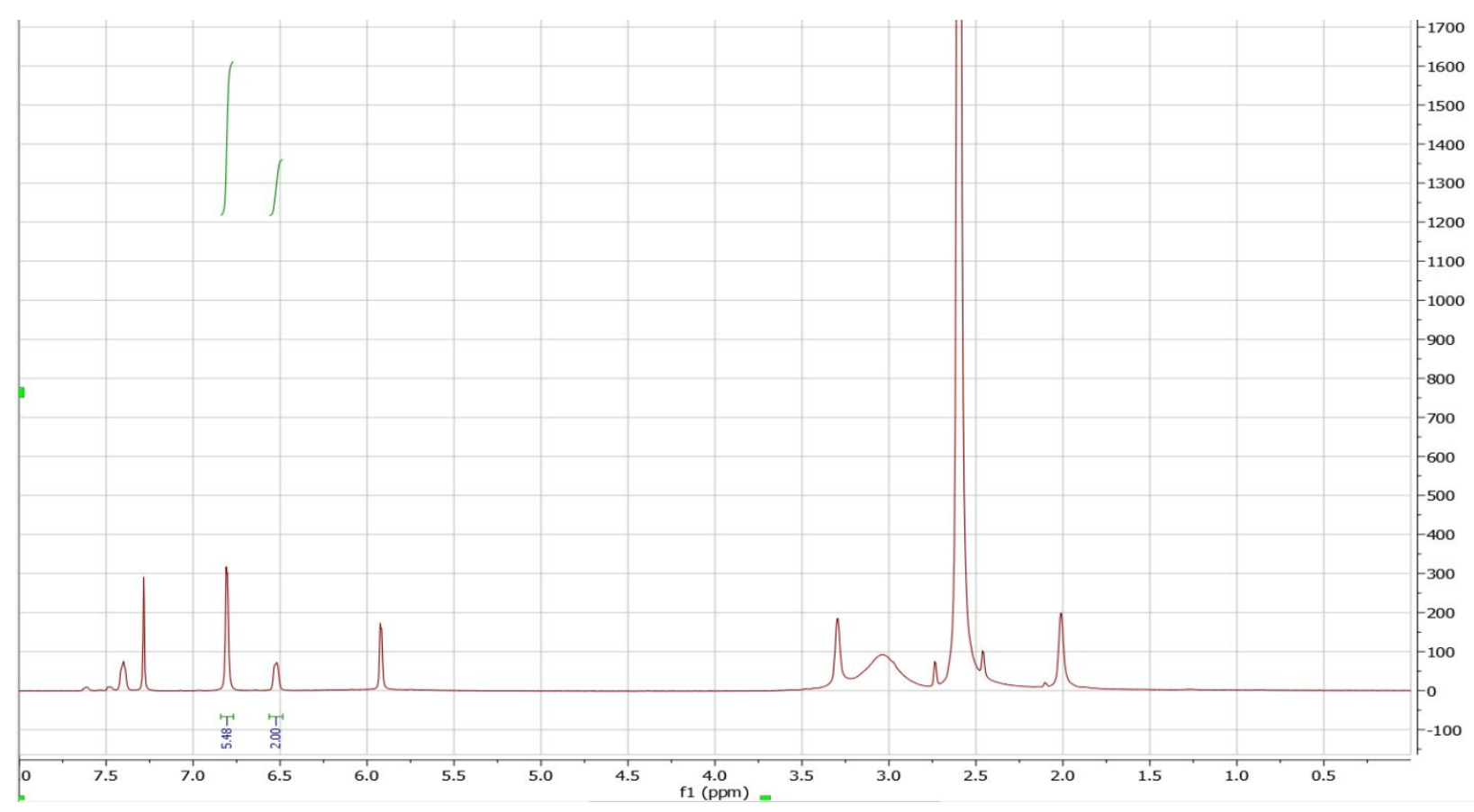

Figure S19: Quantitative ${ }^{1} \mathrm{H}$ NMR for AY calculation of high conversion experiment.

Table S4: Assay yield calculation of high conversion experiments.

\begin{tabular}{|c|c|c|c|}
\hline Reaction mixture & & $\begin{array}{l}\text { 1,3-Benzodioxole added to } \\
\text { sample }\end{array}$ & \\
\hline Sample size (mg) & 2228.0 & $\begin{array}{l}\text { Amount of internal standard } \\
\text { added }(\mathrm{mg})\end{array}$ & 118.5 \\
\hline MW of desired product $(\mathrm{g} / \mathrm{mol})$ & 215.29 & MW of internal standard $(\mathrm{g} / \mathrm{mol})$ & 122.12 \\
\hline Measured integral (6.53 ppm) & 2.00 & Measured integral (6.81 ppm) & 5.48 \\
\hline \# protons & 2 & \# protons & 4 \\
\hline Molar Ratio: & 0.730 & & \\
\hline wt. \% & 6.84 & & \\
\hline Amount collected $(\mathrm{kg})$ & 16.7 & & \\
\hline Amount of desired product $(\mathrm{kg})$ & 1.14 & & \\
\hline $\begin{array}{l}\text { Expected amount of desired } \\
\text { product }(\mathrm{kg})\end{array}$ & 1.27 & & \\
\hline Assay yield & $90.0 \%$ & & \\
\hline Conversion & $90.3 \%$ & & \\
\hline
\end{tabular}




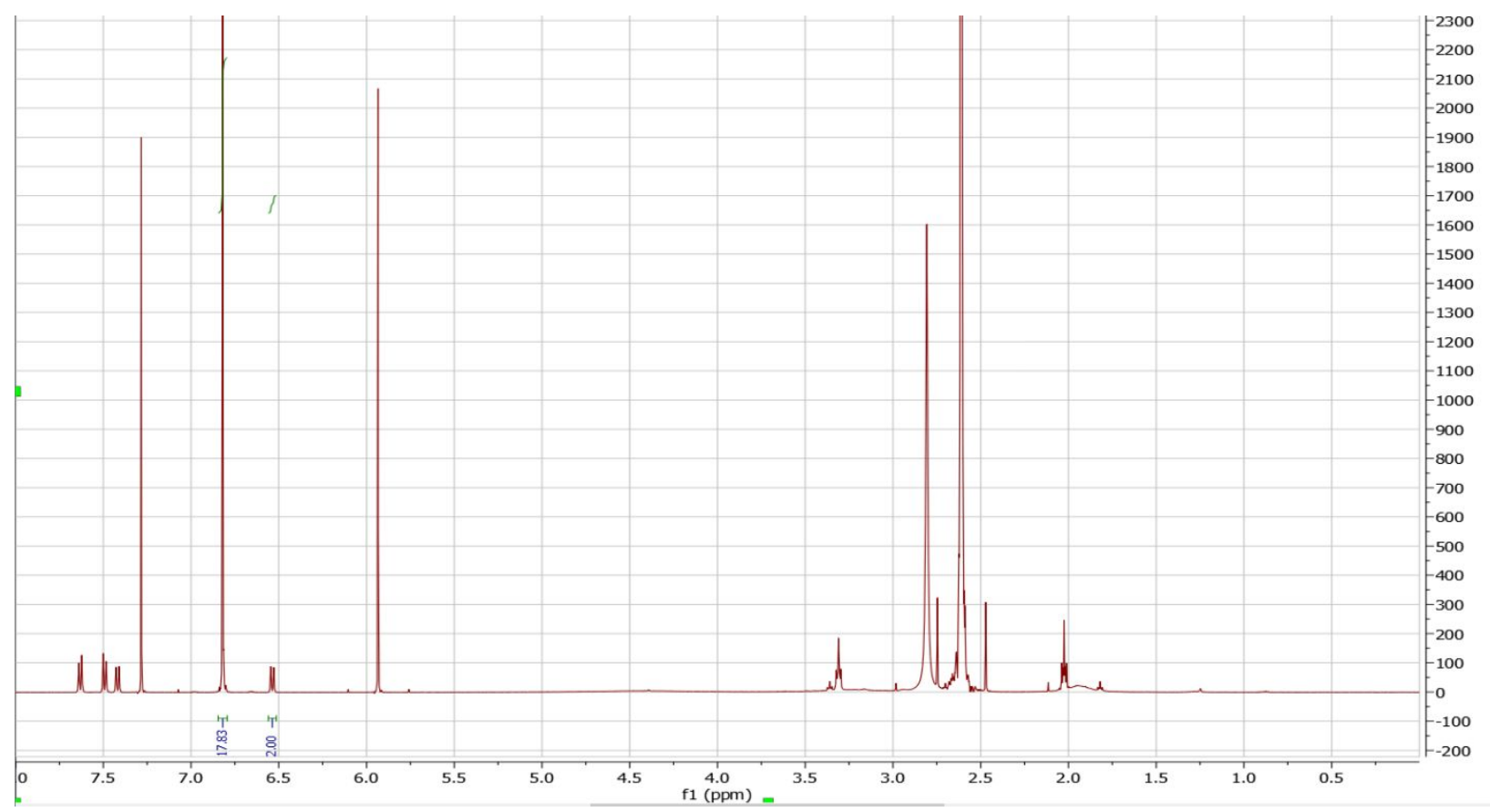

Figure S20: Quantitative ${ }^{1} \mathrm{H}$ NMR for AY calculation of high productivity experiment (first carboy).

Table S5: Assay yield calculation of high productivity experiments (first carboy).

\begin{tabular}{|c|c|c|c|}
\hline Reaction mixture & & $\begin{array}{l}\text { 1,3-Benzodioxole added to } \\
\text { sample }\end{array}$ & \\
\hline Sample size (mg) & 2253.0 & $\begin{array}{l}\text { Amount of internal standard added } \\
\text { (mg) }\end{array}$ & 180.0 \\
\hline MW of desired product $(\mathrm{g} / \mathrm{mol})$ & 215.29 & MW of internal standard $(\mathrm{g} / \mathrm{mol})$ & 122.12 \\
\hline Measure integral (6.53 ppm) & 2 & Measure integral (6.81 ppm) & 17.83 \\
\hline \# protons & 2 & \# protons & 4 \\
\hline Molar Ratio: & 0.224 & & \\
\hline wt. $\%$ & 3.16 & & \\
\hline Amount collected $(\mathrm{kg})$ & 16.8 & & \\
\hline Amount of desired product (kg) & 0.53 & & \\
\hline $\begin{array}{l}\text { Expected amount of desired product } \\
(\mathrm{kg})\end{array}$ & 1.28 & & \\
\hline Assay yield & $41.4 \%$ & & \\
\hline Conversion & $42.0 \%$ & & \\
\hline
\end{tabular}




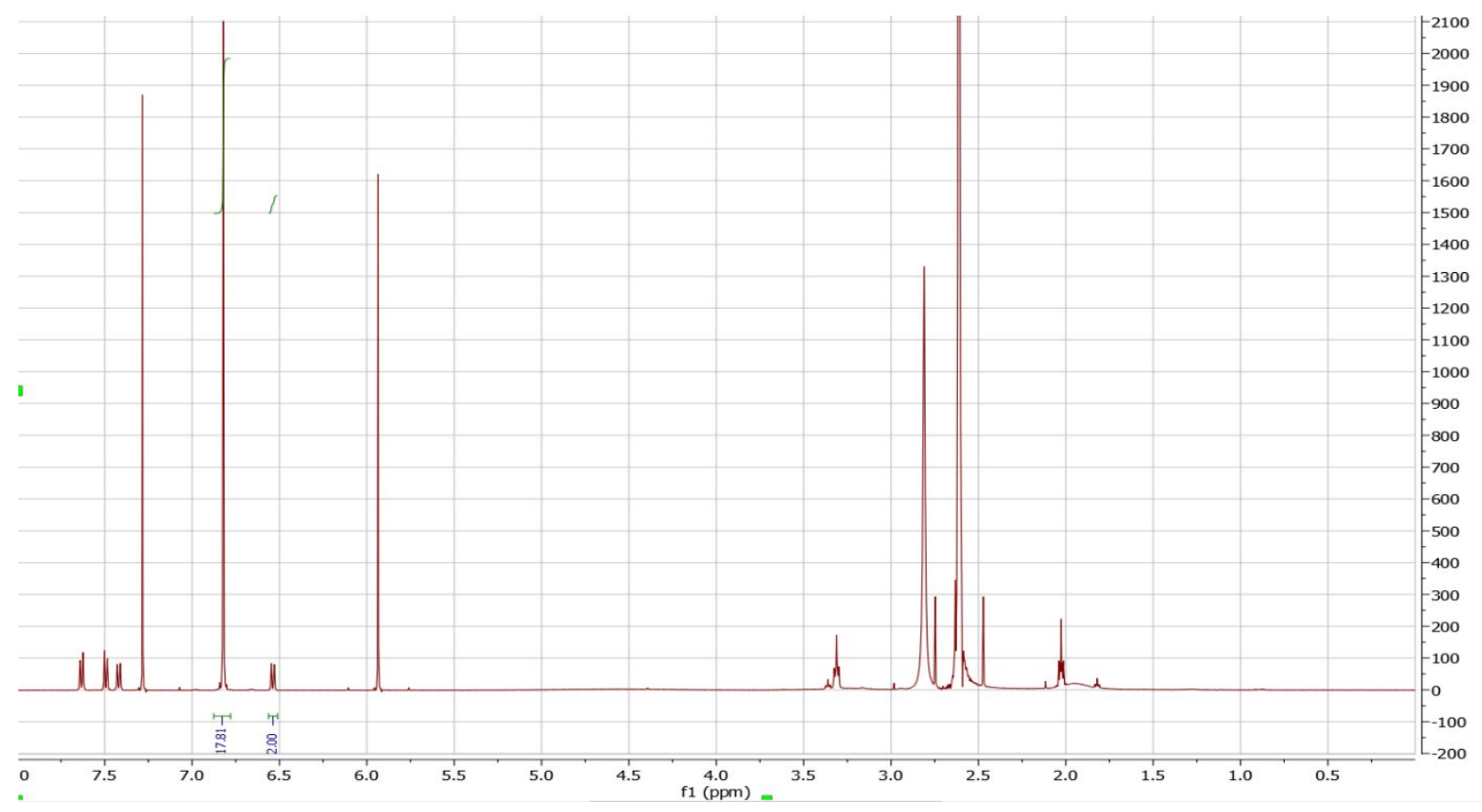

Figure S21: Quantitative ${ }^{1} \mathrm{H}$ NMR for AY calculation of high productivity experiment (second carboy).

Table S6: Assay yield calculation of high productivity experiments (second carboy).

\begin{tabular}{|c|c|c|c|}
\hline Reaction mixture & & $\begin{array}{l}\text { 1,3-Benzodioxole added to } \\
\text { sample }\end{array}$ & \\
\hline Sample size (mg) & 2246.0 & $\begin{array}{l}\text { Amount of internal standard added } \\
(\mathrm{mg})\end{array}$ & 180.0 \\
\hline MW of desired product (g/mol) & 215.29 & MW of internal standard $(\mathrm{g} / \mathrm{mol})$ & 122.12 \\
\hline Measure integral (6.53 ppm) & 2 & Measure integral (6.81 ppm) & 17.81 \\
\hline \# protons & 2 & \# protons & 4 \\
\hline Molar Ratio: & 0.225 & & \\
\hline wt. $\%$ & 3.17 & & \\
\hline Amount collected $(\mathrm{kg})$ & 18.7 & & \\
\hline Amount of desired product $(\mathrm{kg})$ & 0.59 & & \\
\hline $\begin{array}{l}\text { Expected amount of desired product } \\
\text { (kg) }\end{array}$ & 1.42 & & \\
\hline Assay yield & $41.7 \%$ & & \\
\hline Conversion & $42.0 \%$ & & \\
\hline
\end{tabular}




\begin{tabular}{|c|c|c|c|c|c|c|c|}
\hline $\begin{array}{r}\text { time } \\
(h: m m: s s \\
\text { ) }\end{array}$ & $\begin{array}{l}\text { Plate } \\
\text { Right }\end{array}$ & Plate Left & $\begin{array}{l}\text { Reactor } \\
\text { out }\end{array}$ & $\begin{array}{l}\text { Reactor } \\
\text { in }\end{array}$ & $\begin{array}{l}\text { Cooling } \\
\text { line left }\end{array}$ & $\begin{array}{l}\text { Cooling } \\
\text { line right }\end{array}$ & $\begin{array}{l}\text { Water } \\
\text { Bath }\end{array}$ \\
\hline $0: 00: 00$ & 20.2 & 18 & 20 & 19.9 & 20.5 & 20.4 & 18.6 \\
\hline $0: 00: 15$ & 20.2 & 18 & 20 & 19.9 & 20.5 & 20.4 & 18.6 \\
\hline $0: 00: 30$ & 20.3 & 18 & 25.6 & 19.6 & 20.5 & 20.4 & 18.4 \\
\hline $0: 00: 45$ & 20.9 & 16.3 & 27.5 & 20 & 23.1 & 22.7 & 18.8 \\
\hline $0: 01: 00$ & 23 & 13.8 & 29.1 & 19.7 & 25.2 & 24.7 & 18.3 \\
\hline $0: 01: 15$ & 25.1 & 12.2 & 31.5 & 19.6 & 26.4 & 25.8 & 18.4 \\
\hline $0: 01: 30$ & 26.8 & 11.3 & 35 & 19.5 & 27.1 & 26.4 & 18.4 \\
\hline $0: 01: 45$ & 28.1 & 10.8 & 40.1 & 19.5 & 27.5 & 26.8 & 18.3 \\
\hline $0: 02: 00$ & 28.9 & 10.4 & 44.7 & 19.5 & 27.8 & 27.1 & 18.4 \\
\hline $0: 02: 15$ & 29.6 & 10.1 & 48.3 & 19.5 & 28 & 27.3 & 18.6 \\
\hline $0: 02: 30$ & 30 & 10 & 51.1 & 19.5 & 28.1 & 27.4 & 18.6 \\
\hline $0: 02: 45$ & 30.3 & 9.9 & 53.1 & 19.5 & 28.2 & 27.4 & 18.7 \\
\hline $0: 03: 00$ & 30.5 & 9.8 & 54.6 & 19.6 & 28.3 & 27.5 & 18.7 \\
\hline $0: 03: 15$ & 30.7 & 9.8 & 55.6 & 19.6 & 28.3 & 27.5 & 18.8 \\
\hline $0: 03: 30$ & 30.8 & 9.7 & 56.5 & 19.6 & 28.4 & 27.6 & 18.9 \\
\hline $0: 03: 45$ & 30.9 & 9.8 & 57.1 & 19.7 & 28.4 & 27.5 & 18.6 \\
\hline $0: 04: 00$ & 30.9 & 9.7 & 57.5 & 19.7 & 28.5 & 27.6 & 18.8 \\
\hline $0: 04: 15$ & 31 & 9.8 & 58 & 19.7 & 28.4 & 27.6 & 19.1 \\
\hline $0: 04: 30$ & 31 & 10.1 & 58.1 & 19.8 & 27.8 & 27.3 & 18.3 \\
\hline $0: 04: 45$ & 29.2 & 13 & 57.4 & 19.8 & 24.8 & 24.3 & 17.7 \\
\hline $0: 05: 00$ & 26.8 & 15 & 56.5 & 19.8 & 23.2 & 22.8 & 18.6 \\
\hline $0: 05: 15$ & 24.8 & 16.2 & 55.2 & 19.8 & 22.3 & 22 & 18.3 \\
\hline $0: 05: 30$ & 23.4 & 17 & 49.2 & 19.8 & 21.7 & 21.5 & 18.6 \\
\hline $0: 05: 45$ & 22.5 & 17.4 & 39.1 & 19.8 & 21.5 & 21.2 & 19 \\
\hline 0:06:00 & 23.2 & 15.1 & 33.6 & 19.8 & 24.5 & 23.9 & 19.7 \\
\hline $0: 06: 15$ & 25.1 & 13.1 & 31.6 & 19.8 & 26 & 25.4 & 19.9 \\
\hline $0: 06: 30$ & 26.8 & 12 & 30.9 & 19.8 & 26.9 & 26.3 & 19.6 \\
\hline $0: 06: 45$ & 28.2 & 11.2 & 31.1 & 19.8 & 27.4 & 26.7 & 19.5 \\
\hline $0: 07: 00$ & 29.1 & 10.7 & 31.8 & 19.8 & 27.8 & 27.1 & 19.8 \\
\hline $0: 07: 15$ & 29.7 & 10.5 & 32.6 & 19.9 & 28 & 27.3 & 19.8 \\
\hline $0: 07: 30$ & 30.2 & 10.3 & 33.6 & 19.9 & 28.2 & 27.4 & 19.7 \\
\hline $0: 07: 45$ & 30.5 & 10.2 & 34.6 & 19.9 & 28.3 & 27.5 & 19.9 \\
\hline 0:08:00 & 30.7 & 10.1 & 35.4 & 19.9 & 28.4 & 27.6 & 19.9 \\
\hline $0: 08: 15$ & 30.8 & 10.1 & 36.3 & 19.9 & 28.4 & 27.6 & 20.1 \\
\hline $0: 08: 30$ & 30.9 & 10.1 & 37.2 & 19.9 & 28.4 & 27.6 & 20 \\
\hline $0: 08: 45$ & 31 & 10 & 38 & 19.9 & 28.4 & 27.6 & 20.2 \\
\hline $0: 09: 00$ & 31 & 10 & 38.9 & 19.9 & 28.5 & 27.6 & 20.4 \\
\hline $0: 09: 15$ & 31 & 10 & 39.8 & 19.9 & 28.5 & 27.7 & 20.2 \\
\hline $0: 09: 30$ & 31.1 & 10.1 & 40.7 & 19.9 & 28.5 & 27.6 & 20.1 \\
\hline
\end{tabular}

10. STL files data for extended run (All data is in ${ }^{\circ} \mathrm{C}$ ) 


\begin{tabular}{|c|c|c|c|c|c|c|c|}
\hline $0: 09: 45$ & 31.1 & 10.1 & 41.6 & 19.9 & 28.5 & 27.7 & 20.2 \\
\hline $0: 10: 00$ & 31.1 & 10.1 & 42.2 & 19.9 & 28.5 & 27.7 & 20.2 \\
\hline $0: 10: 15$ & 31.2 & 10.1 & 42.8 & 19.9 & 28.5 & 27.7 & 20.2 \\
\hline $0: 10: 30$ & 31.2 & 10.1 & 43.3 & 19.9 & 28.6 & 27.7 & 19.7 \\
\hline $0: 10: 45$ & 31.2 & 10.1 & 43.8 & 19.9 & 28.6 & 27.7 & 19.6 \\
\hline $0: 11: 00$ & 31.2 & 10.1 & 44.3 & 19.9 & 28.5 & 27.7 & 19.9 \\
\hline $0: 11: 15$ & 31.2 & 10.1 & 44.9 & 19.9 & 28.6 & 27.7 & 19.6 \\
\hline $0: 11: 30$ & 31.2 & 10.2 & 45.5 & 19.9 & 28.5 & 27.7 & 19.6 \\
\hline $0: 11: 45$ & 31.3 & 10.2 & 46.2 & 19.9 & 28.6 & 27.7 & 19.4 \\
\hline $0: 12: 00$ & 31.3 & 10.2 & 46.9 & 19.9 & 28.6 & 27.7 & 19.8 \\
\hline $0: 12: 15$ & 31.3 & 10.2 & 47.6 & 19.9 & 28.6 & 27.7 & 19.6 \\
\hline $0: 12: 30$ & 31.2 & 10.2 & 48.3 & 20 & 28.6 & 27.7 & 19.3 \\
\hline $0: 12: 45$ & 31.2 & 10.2 & 49 & 20 & 28.6 & 27.7 & 19.8 \\
\hline $0: 13: 00$ & 31.2 & 10.3 & 49.6 & 20 & 28.6 & 27.7 & 19.3 \\
\hline $0: 13: 15$ & 31.2 & 10.2 & 50.2 & 19.9 & 28.6 & 27.7 & 18.9 \\
\hline $0: 13: 30$ & 31.3 & 10.3 & 50.8 & 19.9 & 28.6 & 27.7 & 18.7 \\
\hline $0: 13: 45$ & 31.3 & 10.2 & 51.4 & 19.9 & 28.6 & 27.7 & 18.8 \\
\hline $0: 14: 00$ & 31.3 & 10.3 & 52 & 19.9 & 28.6 & 27.7 & 18.2 \\
\hline $0: 14: 15$ & 31.3 & 10.2 & 52.5 & 19.9 & 28.6 & 27.7 & 18.5 \\
\hline $0: 14: 30$ & 31.4 & 10.2 & 53.1 & 19.9 & 28.6 & 27.7 & 18.5 \\
\hline $0: 14: 45$ & 31.3 & 10.3 & 53.6 & 19.9 & 28.6 & 27.7 & 18.4 \\
\hline $0: 15: 00$ & 31.3 & 10.3 & 54 & 19.9 & 28.6 & 27.7 & 18.1 \\
\hline $0: 15: 15$ & 31.4 & 10.3 & 54.3 & 19.9 & 28.7 & 27.8 & 18.2 \\
\hline $0: 15: 30$ & 31.4 & 10.3 & 54.7 & 19.9 & 28.6 & 27.7 & 18 \\
\hline $0: 15: 45$ & 31.4 & 10.3 & 54.9 & 19.9 & 28.6 & 27.7 & 18 \\
\hline $0: 16: 00$ & 31.3 & 10.4 & 55.1 & 19.9 & 28.6 & 27.7 & 18.1 \\
\hline $0: 16: 15$ & 31.4 & 10.4 & 55.3 & 19.9 & 28.6 & 27.8 & 18.1 \\
\hline $0: 16: 30$ & 31.4 & 10.4 & 55.4 & 19.9 & 28.6 & 27.7 & 18.1 \\
\hline $0: 16: 45$ & 31.4 & 10.4 & 55.5 & 19.9 & 28.6 & 27.7 & 18 \\
\hline $0: 17: 00$ & 31.4 & 10.4 & 55.6 & 19.9 & 28.6 & 27.7 & 18 \\
\hline $0: 17: 15$ & 31.4 & 10.4 & 55.7 & 19.9 & 28.6 & 27.7 & 17.7 \\
\hline $0: 17: 30$ & 31.4 & 10.5 & 55.7 & 19.9 & 28.6 & 27.7 & 17.7 \\
\hline $0: 17: 45$ & 31.4 & 10.5 & 55.9 & 19.9 & 28.6 & 27.7 & 17.9 \\
\hline $0: 18: 00$ & 31.4 & 10.5 & 55.9 & 19.9 & 28.6 & 27.7 & 17.5 \\
\hline $0: 18: 15$ & 31.4 & 10.5 & 55.9 & 19.9 & 28.6 & 27.8 & 17.6 \\
\hline $0: 18: 30$ & 31.4 & 10.5 & 56 & 19.9 & 28.6 & 27.7 & 17.7 \\
\hline $0: 18: 45$ & 31.4 & 10.5 & 56.1 & 19.9 & 28.6 & 27.7 & 17.7 \\
\hline $0: 19: 00$ & 31.4 & 10.6 & 56.1 & 19.9 & 28.6 & 27.7 & 17.8 \\
\hline $0: 19: 15$ & 31.4 & 10.6 & 56.1 & 19.9 & 28.6 & 27.7 & 17.6 \\
\hline $0: 19: 30$ & 31.5 & 10.6 & 56.1 & 19.9 & 28.6 & 27.8 & 17.6 \\
\hline $0: 19: 45$ & 31.4 & 10.7 & 54.8 & 20 & 28.5 & 27.6 & 17.1 \\
\hline $0: 20: 00$ & 29.7 & 13.4 & 51.5 & 20.1 & 25.2 & 24.5 & 17 \\
\hline $0: 20: 15$ & 27.2 & 15.5 & 50.4 & 20.2 & 23.6 & 23.1 & 16.7 \\
\hline
\end{tabular}




\begin{tabular}{|c|c|c|c|c|c|c|c|}
\hline $0: 20: 30$ & 25.2 & 16.8 & 49.6 & 20.2 & 22.5 & 22.1 & 15.4 \\
\hline $0: 20: 45$ & 23.8 & 17.6 & 48.9 & 20.3 & 21.9 & 21.6 & 18.4 \\
\hline $0: 21: 00$ & 22.7 & 18.1 & 48.2 & 20.3 & 21.5 & 21.2 & 18.2 \\
\hline $0: 21: 15$ & 22 & 18.4 & 47.5 & 20.4 & 21.2 & 21 & 17 \\
\hline $0: 21: 30$ & 21.5 & 18.6 & 46.9 & 20.4 & 21 & 20.8 & 17.6 \\
\hline $0: 21: 45$ & 21.2 & 18.7 & 46.3 & 20.4 & 20.9 & 20.8 & 17.9 \\
\hline $0: 22: 00$ & 21 & 18.7 & 45.7 & 20.4 & 20.8 & 20.7 & 18 \\
\hline $0: 22: 15$ & 20.8 & 18.7 & 45.2 & 20.4 & 20.8 & 20.7 & 17.8 \\
\hline $0: 22: 30$ & 20.6 & 18.7 & 44.6 & 20.4 & 20.7 & 20.6 & 17.8 \\
\hline $0: 22: 45$ & 20.6 & 18.8 & 44 & 20.4 & 20.7 & 20.6 & 17.5 \\
\hline $0: 23: 00$ & 20.5 & 18.8 & 43.4 & 20.4 & 20.7 & 20.6 & 17.4 \\
\hline $0: 23: 15$ & 20.5 & 18.8 & 42.7 & 20.4 & 20.6 & 20.6 & 17.1 \\
\hline $0: 23: 30$ & 20.5 & 18.8 & 42.2 & 20.4 & 20.6 & 20.5 & 16.9 \\
\hline $0: 23: 45$ & 20.4 & 18.7 & 41.6 & 20.4 & 20.6 & 20.5 & 16.8 \\
\hline $0: 24: 00$ & 20.4 & 18.7 & 41.1 & 20.4 & 20.6 & 20.5 & 16.3 \\
\hline $0: 24: 15$ & 20.4 & 18.7 & 40.6 & 20.4 & 20.6 & 20.5 & 16.2 \\
\hline $0: 24: 30$ & 20.4 & 18.7 & 40.1 & 20.4 & 20.6 & 20.5 & 15.8 \\
\hline $0: 24: 45$ & 20.4 & 18.7 & 39.7 & 20.4 & 20.6 & 20.5 & 15.8 \\
\hline $0: 25: 00$ & 20.4 & 18.7 & 39.3 & 20.3 & 20.6 & 20.5 & 15.4 \\
\hline $0: 25: 15$ & 20.4 & 18.7 & 38.8 & 20.3 & 20.6 & 20.5 & 15.1 \\
\hline $0: 25: 30$ & 20.4 & 18.7 & 38.5 & 20.3 & 20.6 & 20.5 & 14.9 \\
\hline $0: 25: 45$ & 20.3 & 18.6 & 38.1 & 20.3 & 20.6 & 20.5 & 14.7 \\
\hline $0: 26: 00$ & 20.3 & 18.6 & 37.7 & 20.3 & 20.6 & 20.5 & 14.2 \\
\hline $0: 26: 15$ & 20.3 & 18.6 & 37.3 & 20.3 & 20.6 & 20.5 & 13.8 \\
\hline $0: 26: 30$ & 20.3 & 18.6 & 36.9 & 20.3 & 20.6 & 20.5 & 14 \\
\hline $0: 26: 45$ & 20.3 & 18.6 & 36.5 & 20.3 & 20.6 & 20.5 & 13.9 \\
\hline $0: 27: 00$ & 20.3 & 18.6 & 36.2 & 20.3 & 20.5 & 20.5 & 13.6 \\
\hline $0: 27: 15$ & 20.3 & 18.5 & 35.8 & 20.3 & 20.6 & 20.5 & 13.2 \\
\hline $0: 27: 30$ & 20.3 & 18.5 & 35.4 & 20.2 & 20.6 & 20.5 & 13 \\
\hline $0: 27: 45$ & 20.3 & 18.5 & 35.1 & 20.2 & 20.5 & 20.5 & 12.9 \\
\hline $0: 28: 00$ & 20.3 & 18.5 & 34.7 & 20.2 & 20.5 & 20.5 & 12.5 \\
\hline $0: 28: 15$ & 20.3 & 18.3 & 34.3 & 19.6 & 20.9 & 20.6 & 12.7 \\
\hline $0: 28: 30$ & 21.9 & 15.6 & 24.6 & 20.2 & 24.1 & 23.7 & 12.7 \\
\hline $0: 28: 45$ & 24.3 & 13.6 & 25.1 & 19.9 & 25.7 & 25.2 & 12.5 \\
\hline $0: 29: 00$ & 26.4 & 12.3 & 26 & 19.7 & 26.7 & 26.1 & 12.7 \\
\hline $0: 29: 15$ & 27.9 & 11.5 & 26.8 & 19.7 & 27.3 & 26.7 & 12.9 \\
\hline $0: 29: 30$ & 28.9 & 11 & 27.8 & 19.7 & 27.7 & 27 & 12.8 \\
\hline $0: 29: 45$ & 29.5 & 10.6 & 28.7 & 19.7 & 28 & 27.2 & 12.8 \\
\hline $0: 30: 00$ & 30 & 10.4 & 29.5 & 19.7 & 28.1 & 27.3 & 13 \\
\hline $0: 30: 15$ & 30.3 & 10.2 & 30.2 & 19.7 & 28.2 & 27.4 & 13.2 \\
\hline $0: 30: 30$ & 30.6 & 10.2 & 30.8 & 19.8 & 28.3 & 27.5 & 13.1 \\
\hline $0: 30: 45$ & 30.7 & 10.1 & 31.3 & 19.8 & 28.4 & 27.6 & 13.2 \\
\hline $0: 31: 00$ & 30.9 & 10.1 & 31.6 & 19.8 & 28.4 & 27.6 & 13.3 \\
\hline
\end{tabular}




\begin{tabular}{|c|c|c|c|c|c|c|c|}
\hline $0: 31: 15$ & 31 & 10 & 32 & 19.8 & 28.5 & 27.6 & 13.3 \\
\hline $0: 31: 30$ & 31 & 10 & 32.4 & 19.9 & 28.5 & 27.6 & 13.5 \\
\hline $0: 31: 45$ & 31 & 10 & 32.7 & 19.9 & 28.5 & 27.6 & 13.5 \\
\hline $0: 32: 00$ & 31.1 & 10 & 32.9 & 19.9 & 28.5 & 27.6 & 13.6 \\
\hline $0: 32: 15$ & 31.1 & 10.1 & 33.2 & 19.9 & 28.5 & 27.6 & 13.7 \\
\hline $0: 32: 30$ & 31.2 & 10.1 & 33.4 & 19.9 & 28.5 & 27.7 & 13.5 \\
\hline $0: 32: 45$ & 31.3 & 10.1 & 33.7 & 19.9 & 28.6 & 27.7 & 13.6 \\
\hline $0: 33: 00$ & 31.3 & 10.1 & 33.8 & 19.9 & 28.6 & 27.7 & 13.7 \\
\hline $0: 33: 15$ & 31.3 & 10.1 & 34 & 19.9 & 28.5 & 27.7 & 13.7 \\
\hline $0: 33: 30$ & 31.3 & 10.1 & 34.2 & 19.9 & 28.6 & 27.7 & 13.8 \\
\hline $0: 33: 45$ & 31.3 & 10.2 & 34.4 & 19.9 & 28.6 & 27.7 & 13.8 \\
\hline $0: 34: 00$ & 31.4 & 10.2 & 34.5 & 19.9 & 28.5 & 27.7 & 13.7 \\
\hline $0: 34: 15$ & 31.4 & 10.2 & 34.6 & 19.9 & 28.5 & 27.7 & 13.5 \\
\hline $0: 34: 30$ & 31.3 & 10.2 & 34.8 & 19.9 & 28.6 & 27.7 & 13.8 \\
\hline $0: 34: 45$ & 31.4 & 10.2 & 34.9 & 19.9 & 28.6 & 27.7 & 13.7 \\
\hline $0: 35: 00$ & 31.4 & 10.2 & 35 & 19.9 & 28.6 & 27.7 & 13.7 \\
\hline $0: 35: 15$ & 31.4 & 10.2 & 35.2 & 19.9 & 28.6 & 27.7 & 13.8 \\
\hline $0: 35: 30$ & 31.4 & 10.2 & 35.3 & 19.9 & 28.6 & 27.8 & 13.7 \\
\hline $0: 35: 45$ & 31.4 & 10.3 & 35.4 & 19.9 & 28.6 & 27.7 & 13.8 \\
\hline $0: 36: 00$ & 31.4 & 10.3 & 35.6 & 19.9 & 28.6 & 27.7 & 13.8 \\
\hline $0: 36: 15$ & 31.4 & 10.3 & 35.7 & 19.9 & 28.6 & 27.7 & 13.8 \\
\hline $0: 36: 30$ & 31.4 & 10.3 & 35.8 & 19.9 & 28.6 & 27.7 & 13.7 \\
\hline $0: 36: 45$ & 31.4 & 10.3 & 35.9 & 19.9 & 28.6 & 27.7 & 14 \\
\hline $0: 37: 00$ & 31.4 & 10.3 & 36 & 19.9 & 28.6 & 27.7 & 13.8 \\
\hline $0: 37: 15$ & 31.4 & 10.3 & 36.2 & 19.9 & 28.6 & 27.7 & 13.8 \\
\hline $0: 37: 30$ & 31.4 & 10.4 & 36.3 & 19.9 & 28.6 & 27.7 & 13.9 \\
\hline $0: 37: 45$ & 31.4 & 10.3 & 36.4 & 19.9 & 28.6 & 27.8 & 13.9 \\
\hline $0: 38: 00$ & 31.5 & 10.4 & 36.5 & 19.9 & 28.6 & 27.7 & 13.9 \\
\hline $0: 38: 15$ & 31.4 & 10.4 & 36.6 & 19.9 & 28.7 & 27.8 & 14.1 \\
\hline $0: 38: 30$ & 31.4 & 10.4 & 36.7 & 19.9 & 28.6 & 27.7 & 14.1 \\
\hline $0: 38: 45$ & 31.5 & 10.5 & 36.9 & 19.9 & 28.6 & 27.7 & 14.1 \\
\hline 0:39:00 & 31.5 & 10.5 & 37 & 19.9 & 28.6 & 27.7 & 14.1 \\
\hline $0: 39: 15$ & 31.4 & 10.5 & 37.1 & 20 & 28.6 & 27.8 & 14.2 \\
\hline $0: 39: 30$ & 31.5 & 10.5 & 37.2 & 20 & 28.6 & 27.7 & 14.2 \\
\hline $0: 39: 45$ & 31.5 & 10.5 & 37.3 & 20 & 28.6 & 27.7 & 14.1 \\
\hline $0: 40: 00$ & 31.5 & 10.5 & 37.4 & 20 & 28.6 & 27.8 & 14.3 \\
\hline $0: 40: 15$ & 31.5 & 10.5 & 37.5 & 19.9 & 28.6 & 27.7 & 14 \\
\hline $0: 40: 30$ & 31.5 & 10.6 & 37.6 & 20 & 28.6 & 27.7 & 14.3 \\
\hline $0: 40: 45$ & 31.5 & 10.5 & 37.7 & 20 & 28.6 & 27.8 & 14.5 \\
\hline $0: 41: 00$ & 31.5 & 10.6 & 37.8 & 20 & 28.6 & 27.7 & 14.3 \\
\hline $0: 41: 15$ & 31.5 & 10.6 & 38 & 20 & 28.6 & 27.8 & 14.5 \\
\hline $0: 41: 30$ & 31.5 & 10.6 & 38 & 20 & 28.6 & 27.8 & 14.5 \\
\hline $0: 41: 45$ & 31.5 & 10.6 & 38.1 & 20 & 28.6 & 27.8 & 14.6 \\
\hline
\end{tabular}




\begin{tabular}{|c|c|c|c|c|c|c|c|}
\hline $0: 42: 00$ & 31.5 & 10.6 & 38.2 & 20 & 28.6 & 27.7 & 14.6 \\
\hline $0: 42: 15$ & 31.5 & 10.6 & 38.3 & 20 & 28.6 & 27.7 & 14.7 \\
\hline $0: 42: 30$ & 31.5 & 10.6 & 38.4 & 20 & 28.6 & 27.7 & 14.7 \\
\hline $0: 42: 45$ & 31.5 & 10.6 & 38.5 & 20 & 28.6 & 27.7 & 15 \\
\hline $0: 43: 00$ & 31.5 & 10.7 & 38.6 & 20 & 28.6 & 27.8 & 14.9 \\
\hline $0: 43: 15$ & 31.5 & 10.7 & 38.7 & 20 & 28.6 & 27.7 & 14.7 \\
\hline $0: 43: 30$ & 31.5 & 10.7 & 38.8 & 20 & 28.7 & 27.8 & 14.9 \\
\hline $0: 43: 45$ & 31.5 & 10.7 & 38.8 & 20 & 28.6 & 27.7 & 15 \\
\hline $0: 44: 00$ & 31.5 & 10.7 & 38.9 & 20 & 28.6 & 27.7 & 14.9 \\
\hline $0: 44: 15$ & 31.5 & 10.7 & 39 & 20 & 28.6 & 27.7 & 14.8 \\
\hline $0: 44: 30$ & 31.5 & 10.7 & 39.1 & 20 & 28.6 & 27.8 & 15.1 \\
\hline $0: 44: 45$ & 31.5 & 10.7 & 39.2 & 20 & 28.6 & 27.8 & 15.1 \\
\hline $0: 45: 00$ & 31.6 & 10.7 & 39.3 & 20 & 28.6 & 27.8 & 15.1 \\
\hline $0: 45: 15$ & 31.5 & 10.7 & 39.4 & 20 & 28.6 & 27.7 & 15.1 \\
\hline $0: 45: 30$ & 31.5 & 10.7 & 39.5 & 20 & 28.6 & 27.7 & 15.2 \\
\hline $0: 45: 45$ & 31.5 & 10.7 & 39.6 & 20 & 28.6 & 27.7 & 15.3 \\
\hline $0: 46: 00$ & 31.5 & 10.7 & 39.7 & 20 & 28.6 & 27.8 & 15.3 \\
\hline $0: 46: 15$ & 31.5 & 10.8 & 39.8 & 20 & 28.6 & 27.8 & 15.4 \\
\hline $0: 46: 30$ & 31.6 & 10.7 & 39.7 & 20 & 28.6 & 27.8 & 15.3 \\
\hline $0: 46: 45$ & 31.6 & 10.7 & 39.9 & 20 & 28.7 & 27.8 & 15.4 \\
\hline $0: 47: 00$ & 31.6 & 10.7 & 39.9 & 20 & 28.6 & 27.8 & 15.4 \\
\hline $0: 47: 15$ & 31.6 & 10.7 & 40 & 20 & 28.6 & 27.7 & 15.5 \\
\hline $0: 47: 30$ & 31.6 & 10.7 & 40.1 & 20 & 28.6 & 27.8 & 15.3 \\
\hline $0: 47: 45$ & 31.5 & 10.7 & 40.2 & 20 & 28.6 & 27.8 & 15.4 \\
\hline $0: 48: 00$ & 31.6 & 10.7 & 40.3 & 20 & 28.6 & 27.8 & 15.4 \\
\hline $0: 48: 15$ & 31.6 & 10.7 & 40.3 & 20 & 28.6 & 27.8 & 15.4 \\
\hline $0: 48: 30$ & 31.6 & 10.7 & 40.5 & 20 & 28.6 & 27.8 & 15.5 \\
\hline $0: 48: 45$ & 31.6 & 10.7 & 40.6 & 20 & 28.7 & 27.8 & 15.5 \\
\hline $0: 49: 00$ & 31.6 & 10.7 & 40.7 & 20 & 28.6 & 27.8 & 15.5 \\
\hline $0: 49: 15$ & 31.6 & 10.7 & 40.8 & 20 & 28.6 & 27.7 & 15.4 \\
\hline $0: 49: 30$ & 31.6 & 10.7 & 40.8 & 20 & 28.6 & 27.8 & 15.3 \\
\hline $0: 49: 45$ & 31.6 & 10.7 & 40.9 & 20 & 28.6 & 27.8 & 15.4 \\
\hline $0: 50: 00$ & 31.6 & 10.7 & 41 & 20 & 28.6 & 27.8 & 15.5 \\
\hline $0: 50: 15$ & 31.6 & 10.7 & 41.1 & 20 & 28.7 & 27.8 & 15.5 \\
\hline $0: 50: 30$ & 31.6 & 10.8 & 41.2 & 20 & 28.6 & 27.7 & 15.4 \\
\hline $0: 50: 45$ & 31.6 & 10.8 & 41.3 & 20 & 28.7 & 27.8 & 15.5 \\
\hline $0: 51: 00$ & 31.6 & 10.7 & 41.4 & 20 & 28.7 & 27.8 & 15.6 \\
\hline $0: 51: 15$ & 31.6 & 10.8 & 41.4 & 19.9 & 28.6 & 27.7 & 15.5 \\
\hline $0: 51: 30$ & 31.6 & 10.8 & 41.5 & 20 & 28.6 & 27.8 & 15.5 \\
\hline $0: 51: 45$ & 31.6 & 10.8 & 41.6 & 20 & 28.6 & 27.8 & 15.5 \\
\hline $0: 52: 00$ & 31.6 & 10.8 & 41.7 & 19.9 & 28.6 & 27.7 & 15.6 \\
\hline $0: 52: 15$ & 31.6 & 10.8 & 41.8 & 20 & 28.7 & 27.8 & 15.6 \\
\hline $0: 52: 30$ & 31.6 & 10.8 & 41.9 & 20 & 28.6 & 27.8 & 15.5 \\
\hline
\end{tabular}




\begin{tabular}{|c|c|c|c|c|c|c|c|}
\hline $0: 52: 45$ & 31.6 & 10.8 & 42 & 20 & 28.6 & 27.7 & 15.6 \\
\hline $0: 53: 00$ & 31.6 & 10.7 & 42 & 20 & 28.7 & 27.8 & 15.5 \\
\hline $0: 53: 15$ & 31.6 & 10.8 & 42.1 & 19.9 & 28.7 & 27.8 & 15.7 \\
\hline $0: 53: 30$ & 31.6 & 10.8 & 42.2 & 20 & 28.6 & 27.7 & 15.7 \\
\hline $0: 53: 45$ & 31.6 & 10.8 & 42.3 & 20 & 28.7 & 27.8 & 15.6 \\
\hline $0: 54: 00$ & 31.6 & 10.8 & 42.3 & 20 & 28.6 & 27.8 & 15.6 \\
\hline $0: 54: 15$ & 31.6 & 10.8 & 42.4 & 20 & 28.7 & 27.8 & 15.8 \\
\hline $0: 54: 30$ & 31.6 & 10.8 & 42.5 & 20 & 28.6 & 27.8 & 15.7 \\
\hline $0: 54: 45$ & 31.6 & 10.8 & 42.5 & 20 & 28.6 & 27.7 & 15.8 \\
\hline $0: 55: 00$ & 31.6 & 10.8 & 42.6 & 19.9 & 28.7 & 27.8 & 15.8 \\
\hline $0: 55: 15$ & 31.6 & 10.8 & 42.7 & 20 & 28.7 & 27.8 & 15.8 \\
\hline $0: 55: 30$ & 31.6 & 10.8 & 42.7 & 20 & 28.7 & 27.8 & 15.9 \\
\hline $0: 55: 45$ & 31.6 & 10.8 & 42.8 & 19.9 & 28.6 & 27.7 & 15.9 \\
\hline $0: 56: 00$ & 31.6 & 10.8 & 42.9 & 20 & 28.7 & 27.8 & 16 \\
\hline $0: 56: 15$ & 31.6 & 10.8 & 42.9 & 20 & 28.7 & 27.8 & 15.9 \\
\hline $0: 56: 30$ & 31.6 & 10.8 & 43 & 20 & 28.6 & 27.7 & 15.9 \\
\hline $0: 56: 45$ & 31.6 & 10.8 & 43.1 & 19.9 & 28.6 & 27.8 & 15.9 \\
\hline $0: 57: 00$ & 31.6 & 10.8 & 43.2 & 20 & 28.7 & 27.8 & 15.9 \\
\hline $0: 57: 15$ & 31.7 & 10.8 & 43.1 & 20 & 28.7 & 27.8 & 16.1 \\
\hline $0: 57: 30$ & 31.7 & 10.8 & 43.1 & 20 & 28.7 & 27.8 & 16 \\
\hline $0: 57: 45$ & 31.7 & 10.8 & 43.2 & 20 & 28.7 & 27.8 & 16 \\
\hline $0: 58: 00$ & 31.7 & 10.8 & 43.2 & 20 & 28.6 & 27.8 & 15.9 \\
\hline $0: 58: 15$ & 31.7 & 10.8 & 43.2 & 20 & 28.7 & 27.8 & 16.2 \\
\hline $0: 58: 30$ & 31.7 & 10.8 & 43.3 & 20 & 28.7 & 27.8 & 16.1 \\
\hline $0: 58: 45$ & 31.7 & 10.9 & 43.4 & 20 & 28.6 & 27.7 & 16.1 \\
\hline 0:59:00 & 31.7 & 10.8 & 43.5 & 20 & 28.7 & 27.9 & 16.1 \\
\hline $0: 59: 15$ & 31.7 & 10.8 & 43.6 & 20 & 28.7 & 27.8 & 16.1 \\
\hline $0: 59: 30$ & 31.7 & 10.8 & 43.7 & 20 & 28.6 & 27.8 & 16.1 \\
\hline $0: 59: 45$ & 31.7 & 10.8 & 43.7 & 20 & 28.7 & 27.8 & 16.2 \\
\hline 1:00:00 & 31.7 & 10.8 & 43.8 & 20 & 28.7 & 27.8 & 16.1 \\
\hline $1: 00: 15$ & 31.7 & 10.9 & 43.9 & 20 & 28.6 & 27.7 & 16.3 \\
\hline $1: 00: 30$ & 31.7 & 10.9 & 43.9 & 20 & 28.7 & 27.8 & 16 \\
\hline $1: 00: 45$ & 31.7 & 10.8 & 44 & 20 & 28.7 & 27.8 & 16.2 \\
\hline 1:01:00 & 31.7 & 10.8 & 44.1 & 20 & 28.7 & 27.8 & 16.1 \\
\hline $1: 01: 15$ & 31.7 & 10.9 & 44.1 & 20 & 28.6 & 27.7 & 16.3 \\
\hline $1: 01: 30$ & 31.7 & 10.9 & 44.2 & 20 & 28.6 & 27.8 & 16.2 \\
\hline $1: 01: 45$ & 31.7 & 10.8 & 44.2 & 20 & 28.7 & 27.8 & 16.3 \\
\hline $1: 02: 00$ & 31.7 & 10.9 & 44.3 & 20.1 & 28.7 & 27.8 & 16.3 \\
\hline $1: 02: 15$ & 31.7 & 10.9 & 44.3 & 20.1 & 28.6 & 27.8 & 16.4 \\
\hline $1: 02: 30$ & 31.7 & 10.9 & 44.4 & 20.1 & 28.7 & 27.8 & 16.2 \\
\hline $1: 02: 45$ & 31.7 & 10.9 & 44.5 & 20.1 & 28.7 & 27.8 & 16.3 \\
\hline 1:03:00 & 31.7 & 10.9 & 44.5 & 20.1 & 28.7 & 27.8 & 16.4 \\
\hline $1: 03: 15$ & 31.7 & 10.8 & 44.6 & 20.1 & 28.7 & 27.8 & 16.2 \\
\hline
\end{tabular}




\begin{tabular}{|c|c|c|c|c|c|c|c|}
\hline $1: 03: 30$ & 31.7 & 10.8 & 44.6 & 20.1 & 28.7 & 27.8 & 16.2 \\
\hline $1: 03: 45$ & 31.7 & 10.8 & 44.7 & 20.1 & 28.6 & 27.8 & 16.2 \\
\hline $1: 04: 00$ & 31.7 & 10.8 & 44.7 & 20.1 & 28.7 & 27.8 & 16.5 \\
\hline $1: 04: 15$ & 31.7 & 10.8 & 44.8 & 20.1 & 28.7 & 27.8 & 16.3 \\
\hline $1: 04: 30$ & 31.7 & 10.8 & 44.8 & 20.1 & 28.7 & 27.8 & 16.5 \\
\hline $1: 04: 45$ & 31.7 & 10.8 & 44.9 & 20.1 & 28.7 & 27.8 & 16.5 \\
\hline $1: 05: 00$ & 31.7 & 10.8 & 44.9 & 20.1 & 28.7 & 27.8 & 16.5 \\
\hline $1: 05: 15$ & 31.7 & 10.8 & 45 & 20 & 28.7 & 27.8 & 16.3 \\
\hline $1: 05: 30$ & 31.7 & 10.8 & 45 & 20.1 & 28.7 & 27.8 & 15.9 \\
\hline $1: 05: 45$ & 31.6 & 10.8 & 45.1 & 20.1 & 28.7 & 27.8 & 16.3 \\
\hline 1:06:00 & 31.6 & 10.8 & 45.2 & 20.1 & 28.7 & 27.8 & 15.9 \\
\hline $1: 06: 15$ & 31.6 & 10.8 & 45.2 & 20.1 & 28.7 & 27.8 & 16 \\
\hline $1: 06: 30$ & 31.6 & 10.8 & 45.2 & 20.1 & 28.7 & 27.8 & 16 \\
\hline $1: 06: 45$ & 31.6 & 10.8 & 45.3 & 20.1 & 28.7 & 27.8 & 16.2 \\
\hline 1:07:00 & 31.6 & 10.9 & 45.3 & 20.1 & 28.7 & 27.8 & 15.9 \\
\hline $1: 07: 15$ & 31.6 & 10.8 & 45.3 & 20.1 & 28.7 & 27.8 & 16 \\
\hline $1: 07: 30$ & 31.6 & 10.8 & 45.4 & 20.1 & 28.7 & 27.8 & 15.8 \\
\hline $1: 07: 45$ & 31.6 & 10.8 & 45.4 & 20.1 & 28.7 & 27.8 & 15.8 \\
\hline $1: 08: 00$ & 31.6 & 10.8 & 45.5 & 20.1 & 28.7 & 27.8 & 15.7 \\
\hline $1: 08: 15$ & 31.6 & 10.8 & 45.5 & 20.1 & 28.7 & 27.8 & 15.6 \\
\hline $1: 08: 30$ & 31.6 & 10.8 & 45.6 & 20.1 & 28.7 & 27.8 & 15.7 \\
\hline $1: 08: 45$ & 31.6 & 10.7 & 45.6 & 20 & 28.7 & 27.8 & 15.7 \\
\hline $1: 09: 00$ & 31.6 & 10.7 & 45.7 & 20 & 28.7 & 27.8 & 15.6 \\
\hline $1: 09: 15$ & 31.6 & 10.7 & 45.7 & 20 & 28.7 & 27.8 & 15.5 \\
\hline $1: 09: 30$ & 31.6 & 10.7 & 45.8 & 20 & 28.7 & 27.8 & 15.4 \\
\hline $1: 09: 45$ & 31.6 & 10.7 & 45.9 & 20 & 28.7 & 27.8 & 15.3 \\
\hline $1: 10: 00$ & 31.6 & 10.7 & 45.9 & 20 & 28.7 & 27.8 & 15.5 \\
\hline $1: 10: 15$ & 31.6 & 10.6 & 45.9 & 20 & 28.7 & 27.8 & 15.5 \\
\hline $1: 10: 30$ & 31.6 & 10.6 & 46 & 20 & 28.7 & 27.8 & 15.4 \\
\hline $1: 10: 45$ & 31.6 & 10.6 & 46 & 20 & 28.7 & 27.8 & 15.3 \\
\hline $1: 11: 00$ & 31.6 & 10.6 & 46 & 20 & 28.7 & 27.8 & 15.3 \\
\hline $1: 11: 15$ & 31.7 & 10.6 & 46.1 & 20 & 28.7 & 27.8 & 15.5 \\
\hline $1: 11: 30$ & 31.7 & 10.6 & 46.2 & 20 & 28.7 & 27.8 & 15.4 \\
\hline $1: 11: 45$ & 31.6 & 10.6 & 46.2 & 20 & 28.7 & 27.8 & 15.7 \\
\hline $1: 12: 00$ & 31.6 & 10.5 & 46.2 & 20 & 28.7 & 27.8 & 15.5 \\
\hline $1: 12: 15$ & 31.7 & 10.6 & 46.3 & 20 & 28.7 & 27.8 & 15.6 \\
\hline $1: 12: 30$ & 31.7 & 10.6 & 46.3 & 20 & 28.7 & 27.8 & 15.5 \\
\hline $1: 12: 45$ & 31.7 & 10.6 & 46.3 & 20 & 28.7 & 27.8 & 15.7 \\
\hline $1: 13: 00$ & 31.7 & 10.6 & 46.4 & 20.1 & 28.7 & 27.8 & 15.6 \\
\hline $1: 13: 15$ & 31.6 & 10.6 & 46.4 & 20.1 & 28.7 & 27.8 & 15.5 \\
\hline $1: 13: 30$ & 31.7 & 10.6 & 46.4 & 20.1 & 28.7 & 27.9 & 15.7 \\
\hline $1: 13: 45$ & 31.7 & 10.6 & 46.5 & 20.1 & 28.7 & 27.8 & 15.7 \\
\hline $1: 14: 00$ & 31.7 & 10.6 & 46.5 & 20.1 & 28.7 & 27.8 & 15.6 \\
\hline
\end{tabular}




\begin{tabular}{|c|c|c|c|c|c|c|c|}
\hline $1: 14: 15$ & 31.6 & 10.6 & 46.6 & 20.1 & 28.7 & 27.8 & 15.8 \\
\hline $1: 14: 30$ & 31.7 & 10.6 & 46.6 & 20.1 & 28.7 & 27.8 & 15.9 \\
\hline $1: 14: 45$ & 31.7 & 10.6 & 46.7 & 20.1 & 28.7 & 27.8 & 15.7 \\
\hline $1: 15: 00$ & 31.7 & 10.6 & 46.7 & 20.1 & 28.7 & 27.8 & 15.8 \\
\hline $1: 15: 15$ & 31.7 & 10.6 & 46.7 & 20.1 & 28.7 & 27.8 & 15.8 \\
\hline $1: 15: 30$ & 31.7 & 10.5 & 46.7 & 20 & 28.7 & 27.9 & 15.8 \\
\hline $1: 15: 45$ & 31.7 & 10.5 & 46.8 & 20 & 28.7 & 27.8 & 15.9 \\
\hline $1: 16: 00$ & 31.7 & 10.5 & 46.8 & 20 & 28.7 & 27.8 & 15.7 \\
\hline $1: 16: 15$ & 31.7 & 10.5 & 46.9 & 20 & 28.7 & 27.8 & 15.9 \\
\hline $1: 16: 30$ & 31.7 & 10.5 & 46.9 & 20 & 28.7 & 27.8 & 16 \\
\hline $1: 16: 45$ & 31.6 & 10.5 & 46.9 & 20 & 28.7 & 27.8 & 16.2 \\
\hline $1: 17: 00$ & 31.6 & 10.5 & 46.9 & 20 & 28.7 & 27.8 & 16.1 \\
\hline $1: 17: 15$ & 31.6 & 10.5 & 47 & 20 & 28.7 & 27.8 & 16.1 \\
\hline $1: 17: 30$ & 31.7 & 10.5 & 47 & 20 & 28.7 & 27.8 & 16 \\
\hline $1: 17: 45$ & 31.7 & 10.5 & 47.1 & 20 & 28.7 & 27.8 & 16.1 \\
\hline $1: 18: 00$ & 31.7 & 10.5 & 47.1 & 20 & 28.7 & 27.8 & 16.2 \\
\hline $1: 18: 15$ & 31.7 & 10.4 & 47.1 & 20 & 28.7 & 27.8 & 16.1 \\
\hline $1: 18: 30$ & 31.7 & 10.4 & 47.1 & 20 & 28.7 & 27.8 & 16.1 \\
\hline $1: 18: 45$ & 31.7 & 10.4 & 47.2 & 20 & 28.7 & 27.8 & 16.3 \\
\hline $1: 19: 00$ & 31.7 & 10.4 & 47.2 & 20 & 28.7 & 27.8 & 16.4 \\
\hline $1: 19: 15$ & 31.7 & 10.4 & 47.2 & 20 & 28.7 & 27.8 & 16.2 \\
\hline $1: 19: 30$ & 31.7 & 10.4 & 47.3 & 20 & 28.7 & 27.8 & 16.4 \\
\hline $1: 19: 45$ & 31.7 & 10.5 & 47.3 & 20.1 & 28.7 & 27.8 & 16.4 \\
\hline $1: 20: 00$ & 31.7 & 10.4 & 47.3 & 20.1 & 28.7 & 27.8 & 16.3 \\
\hline $1: 20: 15$ & 31.7 & 10.5 & 47.4 & 20.1 & 28.7 & 27.8 & 16.3 \\
\hline $1: 20: 30$ & 31.7 & 10.5 & 47.4 & 20.1 & 28.7 & 27.8 & 16.2 \\
\hline $1: 20: 45$ & 31.7 & 10.5 & 47.4 & 20.1 & 28.7 & 27.8 & 16.1 \\
\hline $1: 21: 00$ & 31.7 & 10.5 & 47.4 & 20.1 & 28.7 & 27.8 & 16.3 \\
\hline $1: 21: 15$ & 31.7 & 10.5 & 47.5 & 20.1 & 28.7 & 27.8 & 16.3 \\
\hline $1: 21: 30$ & 31.7 & 10.5 & 47.5 & 20.1 & 28.7 & 27.8 & 16.3 \\
\hline $1: 21: 45$ & 31.7 & 10.5 & 47.6 & 20.1 & 28.7 & 27.8 & 16.3 \\
\hline $1: 22: 00$ & 31.7 & 10.5 & 47.6 & 20.1 & 28.7 & 27.8 & 16.3 \\
\hline $1: 22: 15$ & 31.7 & 10.5 & 47.6 & 20.1 & 28.7 & 27.8 & 16.1 \\
\hline $1: 22: 30$ & 31.7 & 10.5 & 47.6 & 20.1 & 28.7 & 27.8 & 16.2 \\
\hline $1: 22: 45$ & 31.7 & 10.5 & 47.7 & 20.1 & 28.7 & 27.8 & 16.2 \\
\hline $1: 23: 00$ & 31.6 & 10.5 & 47.7 & 20.1 & 28.7 & 27.8 & 15.9 \\
\hline $1: 23: 15$ & 31.6 & 10.5 & 47.7 & 20.1 & 28.7 & 27.8 & 16.1 \\
\hline $1: 23: 30$ & 31.7 & 10.5 & 47.7 & 20 & 28.7 & 27.8 & 16 \\
\hline $1: 23: 45$ & 31.7 & 10.5 & 47.8 & 20.1 & 28.7 & 27.9 & 16.1 \\
\hline $1: 24: 00$ & 31.7 & 10.5 & 47.8 & 20 & 28.7 & 27.8 & 15.9 \\
\hline $1: 24: 15$ & 31.7 & 10.4 & 47.8 & 20 & 28.7 & 27.8 & 16 \\
\hline $1: 24: 30$ & 31.7 & 10.4 & 47.9 & 20 & 28.7 & 27.8 & 16.1 \\
\hline $1: 24: 45$ & 31.7 & 10.4 & 47.9 & 20 & 28.7 & 27.8 & 16 \\
\hline
\end{tabular}




\begin{tabular}{|c|c|c|c|c|c|c|c|}
\hline $1: 25: 00$ & 31.7 & 10.4 & 47.9 & 20 & 28.7 & 27.8 & 16 \\
\hline $1: 25: 15$ & 31.7 & 10.4 & 47.9 & 20 & 28.7 & 27.8 & 16.2 \\
\hline $1: 25: 30$ & 31.7 & 10.4 & 48 & 20 & 28.7 & 27.8 & 16.1 \\
\hline $1: 25: 45$ & 31.7 & 10.4 & 48 & 20 & 28.7 & 27.8 & 16.2 \\
\hline $1: 26: 00$ & 31.7 & 10.4 & 48 & 20 & 28.7 & 27.8 & 16.2 \\
\hline $1: 26: 15$ & 31.7 & 10.4 & 48 & 20 & 28.7 & 27.8 & 16.1 \\
\hline $1: 26: 30$ & 31.6 & 10.4 & 48.1 & 20 & 28.8 & 27.8 & 16.1 \\
\hline $1: 26: 45$ & 31.6 & 10.4 & 48.1 & 20 & 28.7 & 27.9 & 16 \\
\hline $1: 27: 00$ & 31.7 & 10.4 & 48.1 & 20 & 28.7 & 27.8 & 16.1 \\
\hline $1: 27: 15$ & 31.7 & 10.4 & 48.2 & 20 & 28.7 & 27.8 & 16 \\
\hline $1: 27: 30$ & 31.7 & 10.4 & 48.2 & 20 & 28.8 & 27.9 & 16.1 \\
\hline $1: 27: 45$ & 31.7 & 10.4 & 48.2 & 20 & 28.8 & 27.9 & 16.1 \\
\hline $1: 28: 00$ & 31.7 & 10.3 & 48.2 & 20 & 28.8 & 27.9 & 16.1 \\
\hline $1: 28: 15$ & 31.7 & 10.4 & 48.2 & 20 & 28.8 & 27.9 & 16.2 \\
\hline $1: 28: 30$ & 31.7 & 10.3 & 48.3 & 20 & 28.8 & 27.9 & 16.2 \\
\hline $1: 28: 45$ & 31.7 & 10.4 & 48.3 & 20 & 28.7 & 27.8 & 16.2 \\
\hline $1: 29: 00$ & 31.6 & 10.3 & 48.3 & 20 & 28.7 & 27.9 & 16.2 \\
\hline $1: 29: 15$ & 31.7 & 10.3 & 48.3 & 20.1 & 28.7 & 27.9 & 16.2 \\
\hline $1: 29: 30$ & 31.7 & 10.3 & 48.4 & 20.1 & 28.7 & 27.8 & 16.3 \\
\hline $1: 29: 45$ & 31.6 & 10.3 & 48.4 & 20.1 & 28.8 & 27.9 & 16.3 \\
\hline $1: 30: 00$ & 31.6 & 10.3 & 48.4 & 20 & 28.8 & 27.9 & 16.3 \\
\hline $1: 30: 15$ & 31.7 & 10.3 & 48.4 & 20 & 28.8 & 27.8 & 16.4 \\
\hline $1: 30: 30$ & 31.7 & 10.3 & 48.4 & 20 & 28.7 & 27.9 & 16.4 \\
\hline $1: 30: 45$ & 31.6 & 10.2 & 48.5 & 20 & 28.8 & 27.9 & 16.3 \\
\hline $1: 31: 00$ & 31.6 & 10.2 & 48.5 & 20 & 28.7 & 27.8 & 16.4 \\
\hline $1: 31: 15$ & 31.7 & 10.2 & 48.5 & 20 & 28.7 & 27.8 & 16.5 \\
\hline $1: 31: 30$ & 31.7 & 10.2 & 48.5 & 20 & 28.7 & 27.8 & 16.4 \\
\hline $1: 31: 45$ & 31.7 & 10.2 & 48.6 & 20 & 28.8 & 27.8 & 16.5 \\
\hline $1: 32: 00$ & 31.7 & 10.2 & 48.6 & 20 & 28.7 & 27.9 & 16.5 \\
\hline $1: 32: 15$ & 31.6 & 10.2 & 48.6 & 20 & 28.7 & 27.8 & 16.5 \\
\hline $1: 32: 30$ & 31.6 & 10.2 & 48.6 & 20 & 28.7 & 27.8 & 16.5 \\
\hline $1: 32: 45$ & 31.6 & 10.1 & 48.7 & 20 & 28.7 & 27.8 & 16.5 \\
\hline $1: 33: 00$ & 31.6 & 10.1 & 48.7 & 20 & 28.7 & 27.8 & 16.5 \\
\hline $1: 33: 15$ & 31.7 & 10.1 & 48.7 & 20 & 28.7 & 27.8 & 16.5 \\
\hline $1: 33: 30$ & 31.6 & 10.1 & 48.7 & 20 & 28.7 & 27.8 & 16.6 \\
\hline $1: 33: 45$ & 31.7 & 10.1 & 48.7 & 20 & 28.8 & 27.9 & 16.6 \\
\hline $1: 34: 00$ & 31.7 & 10.1 & 48.8 & 20 & 28.8 & 27.8 & 16.6 \\
\hline $1: 34: 15$ & 31.7 & 10.1 & 48.8 & 20 & 28.8 & 27.9 & 16.7 \\
\hline $1: 34: 30$ & 31.7 & 10.1 & 48.8 & 20 & 28.8 & 27.9 & 16.7 \\
\hline $1: 34: 45$ & 31.7 & 10.1 & 48.8 & 20 & 28.7 & 27.8 & 16.6 \\
\hline $1: 35: 00$ & 31.7 & 10.1 & 48.8 & 20 & 28.7 & 27.9 & 16.8 \\
\hline $1: 35: 15$ & 31.6 & 10.1 & 48.9 & 20 & 28.7 & 27.8 & 16.7 \\
\hline $1: 35: 30$ & 31.7 & 10.1 & 48.9 & 20 & 28.7 & 27.8 & 16.8 \\
\hline
\end{tabular}




\begin{tabular}{|c|c|c|c|c|c|c|c|}
\hline $1: 35: 45$ & 31.6 & 10.1 & 48.9 & 20 & 28.7 & 27.8 & 16.7 \\
\hline $1: 36: 00$ & 31.7 & 10 & 48.9 & 20 & 28.8 & 27.9 & 16.8 \\
\hline $1: 36: 15$ & 31.7 & 10.1 & 48.9 & 20 & 28.7 & 27.8 & 16.8 \\
\hline $1: 36: 30$ & 31.6 & 10 & 49 & 20 & 28.8 & 27.9 & 16.9 \\
\hline $1: 36: 45$ & 31.7 & 10 & 49 & 20 & 28.7 & 27.9 & 16.9 \\
\hline $1: 37: 00$ & 31.6 & 10 & 49 & 20 & 28.7 & 27.8 & 16.9 \\
\hline $1: 37: 15$ & 31.6 & 10 & 49 & 20 & 28.8 & 27.8 & 16.9 \\
\hline $1: 37: 30$ & 31.7 & 10 & 49 & 20 & 28.7 & 27.9 & 16.9 \\
\hline $1: 37: 45$ & 31.6 & 10 & 49.1 & 20 & 28.8 & 27.8 & 16.9 \\
\hline $1: 38: 00$ & 31.6 & 10 & 49.1 & 20 & 28.8 & 27.9 & 16.9 \\
\hline $1: 38: 15$ & 31.7 & 10 & 49.1 & 20 & 28.8 & 27.9 & 16.9 \\
\hline $1: 38: 30$ & 31.6 & 10 & 49.1 & 20 & 28.7 & 27.8 & 17 \\
\hline $1: 38: 45$ & 31.6 & 10 & 49.1 & 20 & 28.8 & 27.8 & 17 \\
\hline 1:39:00 & 31.6 & 10 & 49.2 & 20 & 28.8 & 27.9 & 16.9 \\
\hline $1: 39: 15$ & 31.6 & 10 & 49.2 & 20 & 28.8 & 27.8 & 17 \\
\hline $1: 39: 30$ & 31.6 & 10 & 49.2 & 20 & 28.8 & 27.9 & 16.9 \\
\hline $1: 39: 45$ & 31.6 & 10 & 49.2 & 20 & 28.7 & 27.8 & 17 \\
\hline $1: 40: 00$ & 31.7 & 10 & 49.2 & 20 & 28.8 & 27.8 & 16.9 \\
\hline $1: 40: 15$ & 31.7 & 10 & 49.3 & 20 & 28.7 & 27.8 & 17.1 \\
\hline $1: 40: 30$ & 31.8 & 10 & 49.3 & 20 & 28.8 & 27.9 & 17 \\
\hline $1: 40: 45$ & 31.8 & 10 & 49.3 & 20 & 28.8 & 27.9 & 17 \\
\hline $1: 41: 00$ & 31.7 & 10.1 & 49.1 & 20.1 & 28.8 & 27.8 & 17.1 \\
\hline $1: 41: 15$ & 31.7 & 10.1 & 49 & 20.1 & 28.8 & 27.9 & 17.3 \\
\hline $1: 41: 30$ & 31.7 & 10.1 & 49 & 20.1 & 28.7 & 27.8 & 17.1 \\
\hline $1: 41: 45$ & 31.7 & 10.1 & 49 & 20.1 & 28.8 & 27.8 & 17.2 \\
\hline $1: 42: 00$ & 31.7 & 10.1 & 49.1 & 20.1 & 28.7 & 27.9 & 17.2 \\
\hline $1: 42: 15$ & 31.7 & 10.1 & 49.2 & 20.1 & 28.8 & 27.9 & 17.2 \\
\hline $1: 42: 30$ & 31.7 & 10.2 & 49.2 & 20 & 28.8 & 27.8 & 17.2 \\
\hline $1: 42: 45$ & 31.7 & 10.2 & 49.3 & 20 & 28.7 & 27.8 & 17.1 \\
\hline $1: 43: 00$ & 31.7 & 10.2 & 49.3 & 20 & 28.8 & 27.9 & 17.3 \\
\hline $1: 43: 15$ & 31.7 & 10.2 & 49.4 & 20 & 28.8 & 27.8 & 17.2 \\
\hline $1: 43: 30$ & 31.7 & 10.2 & 49.4 & 20 & 28.8 & 27.9 & 17.3 \\
\hline $1: 43: 45$ & 31.7 & 10.2 & 49.4 & 20 & 28.8 & 27.9 & 17.2 \\
\hline $1: 44: 00$ & 31.7 & 10.3 & 49.4 & 20 & 28.7 & 27.8 & 17.3 \\
\hline $1: 44: 15$ & 31.7 & 10.2 & 49.5 & 20 & 28.8 & 27.9 & 17.3 \\
\hline $1: 44: 30$ & 31.8 & 10.3 & 49.5 & 20 & 28.8 & 27.9 & 17.3 \\
\hline $1: 44: 45$ & 31.8 & 10.3 & 49.5 & 20 & 28.7 & 27.8 & 17.4 \\
\hline $1: 45: 00$ & 31.8 & 10.3 & 49.5 & 20 & 28.7 & 27.9 & 17.4 \\
\hline $1: 45: 15$ & 31.8 & 10.3 & 49.5 & 20 & 28.8 & 27.9 & 17.3 \\
\hline $1: 45: 30$ & 31.8 & 10.3 & 49.5 & 20 & 28.8 & 27.8 & 17.3 \\
\hline $1: 45: 45$ & 31.7 & 10.3 & 49.6 & 20 & 28.7 & 27.9 & 17.3 \\
\hline $1: 46: 00$ & 31.7 & 10.3 & 49.6 & 20 & 28.8 & 27.9 & 17.3 \\
\hline $1: 46: 15$ & 31.8 & 10.3 & 49.6 & 20 & 28.8 & 27.8 & 17.3 \\
\hline
\end{tabular}




\begin{tabular}{|c|c|c|c|c|c|c|c|}
\hline $1: 46: 30$ & 31.8 & 10.4 & 49.6 & 20 & 28.7 & 27.9 & 17.4 \\
\hline $1: 46: 45$ & 31.8 & 10.3 & 49.6 & 20 & 28.8 & 27.9 & 17.2 \\
\hline $1: 47: 00$ & 31.8 & 10.4 & 49.6 & 20 & 28.8 & 27.9 & 17.2 \\
\hline $1: 47: 15$ & 31.8 & 10.4 & 49.6 & 20 & 28.8 & 27.8 & 17.3 \\
\hline $1: 47: 30$ & 31.8 & 10.4 & 49.6 & 20 & 28.7 & 27.9 & 17.1 \\
\hline $1: 47: 45$ & 31.8 & 10.4 & 49.6 & 20 & 28.8 & 27.9 & 17 \\
\hline $1: 48: 00$ & 31.8 & 10.4 & 49.6 & 20 & 28.8 & 27.9 & 17.2 \\
\hline $1: 48: 15$ & 31.8 & 10.4 & 49.7 & 20 & 28.8 & 27.9 & 17.2 \\
\hline $1: 48: 30$ & 31.8 & 10.4 & 49.6 & 20 & 28.8 & 27.9 & 17.1 \\
\hline $1: 48: 45$ & 31.8 & 10.4 & 49.7 & 20 & 28.8 & 27.9 & 17.1 \\
\hline $1: 49: 00$ & 31.8 & 10.4 & 49.7 & 20 & 28.8 & 27.9 & 16.8 \\
\hline $1: 49: 15$ & 31.8 & 10.4 & 49.7 & 20 & 28.8 & 27.9 & 16.7 \\
\hline $1: 49: 30$ & 31.8 & 10.4 & 49.7 & 20 & 28.8 & 27.9 & 16.7 \\
\hline $1: 49: 45$ & 31.8 & 10.4 & 49.7 & 20 & 28.8 & 27.9 & 16.7 \\
\hline $1: 50: 00$ & 31.8 & 10.4 & 49.8 & 20 & 28.8 & 27.9 & 16.8 \\
\hline $1: 50: 15$ & 31.8 & 10.4 & 49.8 & 20 & 28.8 & 27.9 & 16.6 \\
\hline $1: 50: 30$ & 31.8 & 10.4 & 49.8 & 20 & 28.8 & 27.9 & 16.7 \\
\hline $1: 50: 45$ & 31.8 & 10.4 & 49.8 & 20 & 28.8 & 27.9 & 16.5 \\
\hline $1: 51: 00$ & 31.8 & 10.5 & 49.9 & 20 & 28.8 & 27.9 & 16.6 \\
\hline $1: 51: 15$ & 31.8 & 10.5 & 49.9 & 20 & 28.8 & 27.9 & 16.5 \\
\hline $1: 51: 30$ & 31.8 & 10.5 & 49.9 & 20 & 28.8 & 27.9 & 16.4 \\
\hline $1: 51: 45$ & 31.8 & 10.5 & 49.9 & 20 & 28.8 & 27.9 & 16.4 \\
\hline $1: 52: 00$ & 31.8 & 10.5 & 49.9 & 20 & 28.8 & 27.9 & 16.4 \\
\hline $1: 52: 15$ & 31.8 & 10.6 & 49.9 & 20 & 28.8 & 27.9 & 16.4 \\
\hline $1: 52: 30$ & 31.8 & 10.6 & 50 & 20 & 28.8 & 27.9 & 16.4 \\
\hline $1: 52: 45$ & 31.8 & 10.6 & 50 & 20 & 28.8 & 27.9 & 16.4 \\
\hline $1: 53: 00$ & 31.8 & 10.6 & 50 & 20 & 28.8 & 27.9 & 16.4 \\
\hline $1: 53: 15$ & 31.8 & 10.6 & 50 & 19.9 & 28.8 & 27.9 & 16.5 \\
\hline $1: 53: 30$ & 31.8 & 10.6 & 50 & 19.9 & 28.8 & 27.9 & 16.5 \\
\hline $1: 53: 45$ & 31.8 & 10.6 & 50 & 19.9 & 28.8 & 27.9 & 16.5 \\
\hline $1: 54: 00$ & 31.8 & 10.6 & 50.1 & 19.9 & 28.8 & 27.9 & 16.4 \\
\hline $1: 54: 15$ & 31.8 & 10.6 & 50.1 & 19.9 & 28.8 & 27.9 & 16.4 \\
\hline $1: 54: 30$ & 31.7 & 10.6 & 50.1 & 19.9 & 28.8 & 27.9 & 16.4 \\
\hline $1: 54: 45$ & 31.8 & 10.6 & 50.1 & 19.9 & 28.8 & 27.9 & 16.4 \\
\hline $1: 55: 00$ & 31.7 & 10.6 & 50.1 & 19.9 & 28.8 & 27.9 & 16.5 \\
\hline $1: 55: 15$ & 31.7 & 10.5 & 50.1 & 19.9 & 28.8 & 27.9 & 16.4 \\
\hline $1: 55: 30$ & 31.7 & 10.5 & 50.1 & 19.9 & 28.8 & 27.9 & 16.5 \\
\hline $1: 55: 45$ & 31.8 & 10.6 & 50.1 & 19.9 & 28.8 & 27.9 & 16.6 \\
\hline $1: 56: 00$ & 31.8 & 10.5 & 50.1 & 19.9 & 28.8 & 27.9 & 16.7 \\
\hline $1: 56: 15$ & 31.8 & 10.5 & 50.2 & 19.9 & 28.8 & 27.9 & 16.7 \\
\hline $1: 56: 30$ & 31.8 & 10.5 & 50.2 & 19.9 & 28.8 & 27.9 & 16.6 \\
\hline $1: 56: 45$ & 31.8 & 10.5 & 50.2 & 19.9 & 28.8 & 27.9 & 16.8 \\
\hline $1: 57: 00$ & 31.8 & 10.5 & 50.2 & 19.9 & 28.8 & 27.9 & 16.7 \\
\hline
\end{tabular}




\begin{tabular}{|c|c|c|c|c|c|c|c|}
\hline $1: 57: 15$ & 31.8 & 10.5 & 50.2 & 19.9 & 28.8 & 27.9 & 16.8 \\
\hline $1: 57: 30$ & 31.8 & 10.4 & 50.2 & 19.9 & 28.8 & 27.9 & 16.7 \\
\hline $1: 57: 45$ & 31.8 & 10.4 & 50.2 & 19.9 & 28.8 & 27.9 & 16.8 \\
\hline $1: 58: 00$ & 31.8 & 10.4 & 50.2 & 19.9 & 28.8 & 27.9 & 16.8 \\
\hline $1: 58: 15$ & 31.8 & 10.4 & 50.3 & 19.9 & 28.8 & 27.9 & 16.9 \\
\hline $1: 58: 30$ & 31.8 & 10.4 & 50.3 & 19.9 & 28.8 & 27.9 & 16.9 \\
\hline $1: 58: 45$ & 31.8 & 10.4 & 50.3 & 19.9 & 28.8 & 27.9 & 17 \\
\hline $1: 59: 00$ & 31.8 & 10.4 & 50.3 & 19.9 & 28.8 & 27.9 & 16.8 \\
\hline $1: 59: 15$ & 31.7 & 10.3 & 50.3 & 19.9 & 28.8 & 27.9 & 16.9 \\
\hline $1: 59: 30$ & 31.7 & 10.3 & 50.3 & 19.9 & 28.8 & 27.9 & 17 \\
\hline $1: 59: 45$ & 31.8 & 10.4 & 50.3 & 19.9 & 28.8 & 27.9 & 17 \\
\hline $2: 00: 00$ & 31.8 & 10.3 & 50.3 & 19.9 & 28.8 & 27.9 & 16.9 \\
\hline $2: 00: 15$ & 31.8 & 10.3 & 50.3 & 19.9 & 28.8 & 27.9 & 17 \\
\hline $2: 00: 30$ & 31.8 & 10.3 & 50.3 & 19.9 & 28.8 & 27.9 & 17 \\
\hline $2: 00: 45$ & 31.8 & 10.3 & 50.3 & 19.9 & 28.8 & 27.9 & 16.9 \\
\hline $2: 01: 00$ & 31.8 & 10.3 & 50.4 & 19.9 & 28.9 & 27.9 & 17 \\
\hline $2: 01: 15$ & 31.8 & 10.3 & 50.4 & 19.9 & 28.8 & 27.9 & 16.9 \\
\hline $2: 01: 30$ & 31.8 & 10.3 & 50.4 & 19.9 & 28.8 & 27.9 & 17 \\
\hline $2: 01: 45$ & 31.8 & 10.3 & 50.4 & 19.9 & 28.8 & 27.9 & 16.9 \\
\hline $2: 02: 00$ & 31.8 & 10.2 & 50.4 & 19.9 & 28.9 & 28 & 17 \\
\hline $2: 02: 15$ & 31.8 & 10.3 & 50.4 & 19.9 & 28.8 & 27.9 & 17 \\
\hline $2: 02: 30$ & 31.8 & 10.3 & 50.4 & 19.9 & 28.8 & 27.9 & 16.9 \\
\hline $2: 02: 45$ & 31.8 & 10.2 & 50.4 & 19.9 & 28.8 & 28 & 17 \\
\hline $2: 03: 00$ & 31.8 & 10.2 & 50.4 & 19.9 & 28.8 & 27.9 & 17 \\
\hline $2: 03: 15$ & 31.8 & 10.3 & 50.4 & 19.9 & 28.8 & 27.9 & 17.1 \\
\hline $2: 03: 30$ & 31.8 & 10.3 & 50.5 & 19.9 & 28.8 & 27.9 & 17 \\
\hline $2: 03: 45$ & 31.8 & 10.3 & 50.5 & 19.9 & 28.8 & 27.9 & 17.2 \\
\hline $2: 04: 00$ & 31.8 & 10.3 & 50.5 & 19.9 & 28.8 & 27.9 & 17 \\
\hline $2: 04: 15$ & 31.8 & 10.2 & 50.5 & 19.9 & 28.8 & 27.9 & 17.1 \\
\hline $2: 04: 30$ & 31.8 & 10.2 & 50.5 & 19.9 & 28.8 & 27.9 & 17.2 \\
\hline $2: 04: 45$ & 31.8 & 10.2 & 50.5 & 19.9 & 28.9 & 27.9 & 17.1 \\
\hline $2: 05: 00$ & 31.9 & 10.2 & 50.5 & 19.9 & 28.8 & 27.9 & 17.3 \\
\hline $2: 05: 15$ & 31.8 & 10.2 & 50.5 & 19.9 & 28.8 & 28 & 17 \\
\hline $2: 05: 30$ & 31.8 & 10.2 & 50.5 & 19.9 & 28.8 & 27.9 & 16.9 \\
\hline $2: 05: 45$ & 31.8 & 10.3 & 50.5 & 19.9 & 28.8 & 27.9 & 17.1 \\
\hline $2: 06: 00$ & 31.7 & 10.3 & 50.5 & 19.9 & 28.8 & 27.9 & 17 \\
\hline $2: 06: 15$ & 31.8 & 10.2 & 50.6 & 20 & 28.9 & 27.9 & 17.1 \\
\hline $2: 06: 30$ & 31.8 & 10.3 & 50.6 & 20 & 28.8 & 27.9 & 17.2 \\
\hline $2: 06: 45$ & 31.8 & 10.2 & 50.6 & 20 & 28.8 & 28 & 16.9 \\
\hline $2: 07: 00$ & 31.8 & 10.2 & 50.6 & 20 & 28.9 & 28 & 17.1 \\
\hline $2: 07: 15$ & 31.8 & 10.2 & 50.5 & 20 & 28.8 & 28 & 17.2 \\
\hline $2: 07: 30$ & 31.8 & 10.3 & 50.6 & 20 & 28.9 & 28 & 17.1 \\
\hline $2: 07: 45$ & 31.8 & 10.3 & 50.6 & 20 & 28.8 & 27.9 & 17.2 \\
\hline
\end{tabular}




\begin{tabular}{|c|c|c|c|c|c|c|c|}
\hline 2:08:00 & 31.8 & 10.2 & 50.6 & 20 & 28.9 & 28 & 17.2 \\
\hline $2: 08: 15$ & 31.8 & 10.2 & 50.6 & 20 & 28.9 & 28 & 17.3 \\
\hline $2: 08: 30$ & 31.8 & 10.2 & 50.6 & 20 & 28.9 & 28 & 17.1 \\
\hline $2: 08: 45$ & 31.8 & 10.3 & 50.7 & 20.1 & 28.8 & 28 & 17.3 \\
\hline $2: 09: 00$ & 31.8 & 10.2 & 50.7 & 20.1 & 28.9 & 28 & 17.4 \\
\hline $2: 09: 15$ & 31.9 & 10.3 & 50.7 & 20.1 & 28.9 & 27.9 & 17.1 \\
\hline $2: 09: 30$ & 31.8 & 10.3 & 50.7 & 20 & 28.9 & 28 & 17.2 \\
\hline $2: 09: 45$ & 31.9 & 10.3 & 50.7 & 20 & 28.9 & 28 & 17.2 \\
\hline $2: 10: 00$ & 31.9 & 10.3 & 50.7 & 20 & 28.9 & 28 & 17.4 \\
\hline $2: 10: 15$ & 31.8 & 10.3 & 50.7 & 20 & 28.9 & 28 & 17.3 \\
\hline $2: 10: 30$ & 31.8 & 10.3 & 50.7 & 20 & 28.9 & 28 & 17.2 \\
\hline $2: 10: 45$ & 31.8 & 10.2 & 50.7 & 20 & 28.9 & 27.9 & 17.4 \\
\hline $2: 11: 00$ & 31.8 & 10.2 & 50.7 & 20 & 28.9 & 28 & 17.1 \\
\hline $2: 11: 15$ & 31.9 & 10.3 & 50.7 & 20 & 28.9 & 28 & 17.3 \\
\hline $2: 11: 30$ & 31.8 & 10.3 & 50.7 & 20 & 28.9 & 27.9 & 17.1 \\
\hline $2: 11: 45$ & 31.9 & 10.3 & 50.7 & 20 & 28.8 & 27.9 & 17.3 \\
\hline $2: 12: 00$ & 31.8 & 10.3 & 50.7 & 20 & 28.9 & 28 & 17.2 \\
\hline $2: 12: 15$ & 31.8 & 10.3 & 50.7 & 20 & 28.9 & 27.9 & 17.3 \\
\hline $2: 12: 30$ & 31.8 & 10.3 & 50.7 & 20 & 28.9 & 28 & 17.4 \\
\hline $2: 12: 45$ & 31.9 & 10.3 & 50.8 & 20 & 28.9 & 28 & 17.1 \\
\hline $2: 13: 00$ & 31.9 & 10.2 & 50.8 & 20 & 28.9 & 28 & 17.4 \\
\hline $2: 13: 15$ & 31.9 & 10.2 & 50.8 & 20 & 28.9 & 27.9 & 17.2 \\
\hline $2: 13: 30$ & 31.9 & 10.3 & 50.8 & 20 & 28.9 & 28 & 17.4 \\
\hline $2: 13: 45$ & 31.8 & 10.2 & 50.8 & 20 & 28.9 & 27.9 & 17.3 \\
\hline $2: 14: 00$ & 31.8 & 10.3 & 50.8 & 20 & 28.9 & 28 & 17.3 \\
\hline $2: 14: 15$ & 31.9 & 10.3 & 50.8 & 20 & 28.9 & 28 & 17.4 \\
\hline $2: 14: 30$ & 31.9 & 10.3 & 50.9 & 20 & 28.9 & 27.9 & 17.3 \\
\hline $2: 14: 45$ & 31.8 & 10.3 & 50.9 & 20 & 28.8 & 27.9 & 17.2 \\
\hline $2: 15: 00$ & 31.8 & 10.3 & 50.9 & 20 & 28.9 & 28 & 17.2 \\
\hline $2: 15: 15$ & 31.8 & 10.3 & 50.9 & 20 & 28.9 & 28 & 17.2 \\
\hline $2: 15: 30$ & 31.8 & 10.3 & 50.9 & 20 & 28.9 & 27.9 & 17.3 \\
\hline $2: 15: 45$ & 31.8 & 10.3 & 50.9 & 20 & 28.9 & 28 & 17.3 \\
\hline $2: 16: 00$ & 31.8 & 10.4 & 50.9 & 20 & 28.9 & 28 & 17.2 \\
\hline $2: 16: 15$ & 31.8 & 10.4 & 50.9 & 20 & 28.9 & 28 & 17.5 \\
\hline $2: 16: 30$ & 31.9 & 10.4 & 50.9 & 20 & 28.9 & 28 & 17.3 \\
\hline $2: 16: 45$ & 31.8 & 10.4 & 50.9 & 20 & 28.9 & 28 & 17.4 \\
\hline $2: 17: 00$ & 31.9 & 10.4 & 50.9 & 20 & 28.9 & 28 & 17.3 \\
\hline $2: 17: 15$ & 31.9 & 10.3 & 50.9 & 20 & 28.9 & 28 & 17.4 \\
\hline $2: 17: 30$ & 31.8 & 10.4 & 50.9 & 20.1 & 28.9 & 27.9 & 17.3 \\
\hline $2: 17: 45$ & 31.8 & 10.4 & 51 & 20 & 28.9 & 27.9 & 17.4 \\
\hline $2: 18: 00$ & 31.9 & 10.4 & 51 & 20 & 28.9 & 28 & 17.4 \\
\hline $2: 18: 15$ & 31.9 & 10.4 & 51 & 20 & 28.9 & 27.9 & 17.3 \\
\hline $2: 18: 30$ & 31.9 & 10.4 & 51 & 20 & 28.9 & 27.9 & 17.4 \\
\hline
\end{tabular}




\begin{tabular}{|c|c|c|c|c|c|c|c|}
\hline $2: 18: 45$ & 31.9 & 10.4 & 51 & 20.1 & 28.8 & 28 & 17.5 \\
\hline $2: 19: 00$ & 31.9 & 10.4 & 51 & 20 & 28.9 & 28 & 17.3 \\
\hline $2: 19: 15$ & 31.9 & 10.4 & 51 & 20 & 28.9 & 28 & 17.4 \\
\hline $2: 19: 30$ & 31.9 & 10.4 & 51 & 20.1 & 28.9 & 28 & 17.5 \\
\hline $2: 19: 45$ & 31.9 & 10.4 & 51 & 20 & 28.9 & 28 & 17.5 \\
\hline $2: 20: 00$ & 31.9 & 10.4 & 51 & 20 & 28.8 & 27.9 & 17.5 \\
\hline $2: 20: 15$ & 31.9 & 10.4 & 51 & 20.1 & 28.8 & 27.9 & 17.4 \\
\hline $2: 20: 30$ & 31.9 & 10.4 & 51 & 20.1 & 28.9 & 28 & 17.4 \\
\hline $2: 20: 45$ & 31.9 & 10.4 & 51 & 20.1 & 28.9 & 27.9 & 17.5 \\
\hline $2: 21: 00$ & 31.8 & 10.4 & 51 & 20.1 & 28.9 & 28 & 17.4 \\
\hline $2: 21: 15$ & 31.9 & 10.4 & 51 & 20.1 & 28.9 & 28 & 17.6 \\
\hline $2: 21: 30$ & 31.9 & 10.4 & 51 & 20.1 & 28.9 & 28 & 17.5 \\
\hline $2: 21: 45$ & 31.9 & 10.5 & 51 & 20.1 & 28.9 & 28 & 17.3 \\
\hline $2: 22: 00$ & 31.9 & 10.4 & 51 & 20.1 & 28.9 & 28 & 17.2 \\
\hline $2: 22: 15$ & 31.9 & 10.4 & 51 & 20.1 & 28.9 & 28 & 17.6 \\
\hline $2: 22: 30$ & 31.9 & 10.5 & 51 & 20.1 & 28.8 & 27.9 & 17.4 \\
\hline $2: 22: 45$ & 31.9 & 10.5 & 51 & 20.1 & 28.8 & 27.9 & 17.4 \\
\hline $2: 23: 00$ & 31.9 & 10.5 & 51.1 & 20.1 & 28.9 & 28 & 17.2 \\
\hline $2: 23: 15$ & 31.9 & 10.5 & 51.1 & 20.1 & 28.9 & 28 & 17.4 \\
\hline $2: 23: 30$ & 31.9 & 10.5 & 51.1 & 20.1 & 28.9 & 28 & 17.4 \\
\hline $2: 23: 45$ & 31.9 & 10.5 & 51.1 & 20.1 & 28.9 & 27.9 & 17.1 \\
\hline $2: 24: 00$ & 31.9 & 10.5 & 51.1 & 20.1 & 28.9 & 27.9 & 17.2 \\
\hline $2: 24: 15$ & 31.9 & 10.5 & 51.1 & 20.1 & 28.9 & 28 & 16.9 \\
\hline $2: 24: 30$ & 31.9 & 10.5 & 51.1 & 20.1 & 28.9 & 27.9 & 17.1 \\
\hline $2: 24: 45$ & 31.9 & 10.6 & 51.1 & 20.1 & 28.9 & 28 & 17.1 \\
\hline $2: 25: 00$ & 31.9 & 10.6 & 51.1 & 20.1 & 28.9 & 27.9 & 16.9 \\
\hline $2: 25: 15$ & 31.9 & 10.6 & 51.1 & 20.1 & 28.8 & 27.9 & 17 \\
\hline $2: 25: 30$ & 31.9 & 10.6 & 51.1 & 20.1 & 28.9 & 28 & 16.8 \\
\hline $2: 25: 45$ & 31.9 & 10.6 & 51.1 & 20.1 & 28.9 & 28 & 16.7 \\
\hline $2: 26: 00$ & 31.8 & 10.5 & 51.1 & 20.1 & 28.9 & 27.9 & 16.7 \\
\hline $2: 26: 15$ & 31.9 & 10.6 & 51.2 & 20.1 & 28.8 & 27.9 & 16.9 \\
\hline $2: 26: 30$ & 31.9 & 10.5 & 51.1 & 20.1 & 28.8 & 27.9 & 16.7 \\
\hline $2: 26: 45$ & 31.8 & 10.5 & 51.1 & 20.1 & 28.8 & 27.9 & 16.7 \\
\hline $2: 27: 00$ & 31.9 & 10.5 & 51.1 & 20 & 28.8 & 27.9 & 16.8 \\
\hline $2: 27: 15$ & 31.9 & 10.5 & 51.1 & 20 & 28.9 & 28 & 16.7 \\
\hline $2: 27: 30$ & 31.9 & 10.5 & 51.1 & 20 & 28.8 & 27.9 & 16.7 \\
\hline $2: 27: 45$ & 31.9 & 10.5 & 51.1 & 20 & 28.8 & 27.9 & 16.7 \\
\hline $2: 28: 00$ & 31.8 & 10.5 & 51.1 & 20 & 28.8 & 27.9 & 16.6 \\
\hline $2: 28: 15$ & 31.8 & 10.4 & 51.1 & 20 & 28.8 & 27.9 & 16.7 \\
\hline $2: 28: 30$ & 31.8 & 10.4 & 51.1 & 20 & 28.9 & 27.9 & 16.8 \\
\hline $2: 28: 45$ & 31.8 & 10.4 & 51.2 & 20 & 28.8 & 27.9 & 16.8 \\
\hline $2: 29: 00$ & 31.8 & 10.4 & 51.2 & 20 & 28.8 & 27.9 & 16.8 \\
\hline $2: 29: 15$ & 31.8 & 10.4 & 51.2 & 20 & 28.9 & 27.9 & 16.8 \\
\hline
\end{tabular}




\begin{tabular}{|c|c|c|c|c|c|c|c|}
\hline $2: 29: 30$ & 31.8 & 10.4 & 51.2 & 20 & 28.8 & 27.9 & 16.8 \\
\hline $2: 29: 45$ & 31.8 & 10.3 & 51.2 & 20 & 28.8 & 27.9 & 16.8 \\
\hline $2: 30: 00$ & 31.8 & 10.3 & 51.2 & 20 & 28.8 & 27.9 & 16.9 \\
\hline $2: 30: 15$ & 31.8 & 10.3 & 51.2 & 20 & 28.8 & 27.9 & 17 \\
\hline $2: 30: 30$ & 31.8 & 10.3 & 51.2 & 20 & 28.8 & 27.9 & 16.9 \\
\hline $2: 30: 45$ & 31.8 & 10.3 & 51.2 & 20 & 28.8 & 27.9 & 16.9 \\
\hline $2: 31: 00$ & 31.8 & 10.3 & 51.2 & 20 & 28.8 & 27.9 & 17 \\
\hline $2: 31: 15$ & 31.8 & 10.3 & 51.2 & 19.9 & 28.9 & 28 & 16.8 \\
\hline $2: 31: 30$ & 31.8 & 10.3 & 51.2 & 19.9 & 28.8 & 27.9 & 17 \\
\hline $2: 31: 45$ & 31.8 & 10.3 & 51.2 & 19.9 & 28.8 & 28 & 16.9 \\
\hline $2: 32: 00$ & 31.8 & 10.3 & 51.2 & 19.9 & 28.8 & 27.9 & 17.1 \\
\hline $2: 32: 15$ & 31.8 & 10.2 & 51.2 & 19.9 & 28.9 & 27.9 & 16.9 \\
\hline $2: 32: 30$ & 31.9 & 10.2 & 51.2 & 19.9 & 28.8 & 27.9 & 17 \\
\hline $2: 32: 45$ & 31.9 & 10.2 & 51.2 & 19.9 & 28.9 & 27.9 & 16.9 \\
\hline $2: 33: 00$ & 31.8 & 10.2 & 51.2 & 19.9 & 28.8 & 27.9 & 17.1 \\
\hline $2: 33: 15$ & 31.8 & 10.2 & 51.2 & 19.9 & 28.8 & 28 & 17.1 \\
\hline $2: 33: 30$ & 31.8 & 10.2 & 51.2 & 19.9 & 28.8 & 27.9 & 17 \\
\hline $2: 33: 45$ & 31.8 & 10.2 & 51.2 & 19.9 & 28.8 & 27.9 & 17 \\
\hline $2: 34: 00$ & 31.8 & 10.2 & 51.3 & 19.9 & 28.8 & 27.9 & 17 \\
\hline $2: 34: 15$ & 31.8 & 10.2 & 51.3 & 20 & 28.8 & 27.9 & 17 \\
\hline $2: 34: 30$ & 31.8 & 10.2 & 51.3 & 20 & 28.8 & 27.9 & 17.1 \\
\hline $2: 34: 45$ & 31.8 & 10.2 & 51.3 & 20 & 28.8 & 27.9 & 17.2 \\
\hline $2: 35: 00$ & 31.8 & 10.2 & 51.3 & 20 & 28.9 & 27.9 & 17.1 \\
\hline $2: 35: 15$ & 31.8 & 10.2 & 51.3 & 20 & 28.8 & 27.9 & 17.2 \\
\hline $2: 35: 30$ & 31.9 & 10.2 & 51.3 & 20 & 28.9 & 28 & 17.2 \\
\hline $2: 35: 45$ & 31.9 & 10.3 & 51.3 & 20 & 28.8 & 27.9 & 17.1 \\
\hline $2: 36: 00$ & 31.9 & 10.3 & 51.3 & 20 & 28.8 & 27.9 & 17.2 \\
\hline $2: 36: 15$ & 31.9 & 10.3 & 51.4 & 20.1 & 28.9 & 27.9 & 17.2 \\
\hline $2: 36: 30$ & 31.9 & 10.3 & 51.3 & 20.1 & 28.9 & 27.9 & 17.1 \\
\hline $2: 36: 45$ & 31.9 & 10.4 & 51.3 & 20.1 & 28.9 & 27.9 & 17.2 \\
\hline $2: 37: 00$ & 31.9 & 10.4 & 51.4 & 20.1 & 28.8 & 27.9 & 17.3 \\
\hline $2: 37: 15$ & 31.9 & 10.4 & 51.4 & 20.1 & 28.9 & 28 & 17.2 \\
\hline $2: 37: 30$ & 31.9 & 10.4 & 51.4 & 20.1 & 28.8 & 27.9 & 17.3 \\
\hline $2: 37: 45$ & 31.9 & 10.5 & 51.4 & 20.1 & 28.9 & 27.9 & 17.3 \\
\hline $2: 38: 00$ & 31.9 & 10.5 & 51.4 & 20.1 & 28.8 & 27.9 & 17.3 \\
\hline $2: 38: 15$ & 31.9 & 10.5 & 51.4 & 20.1 & 28.8 & 27.9 & 17.3 \\
\hline $2: 38: 30$ & 31.9 & 10.5 & 51.4 & 20.1 & 28.8 & 27.9 & 17.3 \\
\hline $2: 38: 45$ & 31.9 & 10.5 & 51.4 & 20.1 & 28.8 & 28 & 17.4 \\
\hline $2: 39: 00$ & 32 & 10.6 & 51.4 & 20.1 & 28.8 & 27.9 & 17.3 \\
\hline $2: 39: 15$ & 31.9 & 10.6 & 51.4 & 20.1 & 28.9 & 27.9 & 17.4 \\
\hline $2: 39: 30$ & 31.9 & 10.6 & 51.4 & 20.1 & 28.9 & 28 & 17.5 \\
\hline $2: 39: 45$ & 31.9 & 10.6 & 51.4 & 20.1 & 28.9 & 27.9 & 17.5 \\
\hline $2: 40: 00$ & 31.9 & 10.6 & 51.5 & 20.1 & 28.9 & 27.9 & 17.3 \\
\hline
\end{tabular}




\begin{tabular}{|c|c|c|c|c|c|c|c|}
\hline $2: 40: 15$ & 31.9 & 10.7 & 51.5 & 20.1 & 28.9 & 28 & 17.4 \\
\hline $2: 40: 30$ & 31.9 & 10.7 & 51.5 & 20.1 & 28.9 & 27.9 & 17.3 \\
\hline $2: 40: 45$ & 31.9 & 10.7 & 51.5 & 20.1 & 28.8 & 27.9 & 17.5 \\
\hline $2: 41: 00$ & 31.9 & 10.8 & 51.5 & 20.1 & 28.8 & 27.9 & 17.4 \\
\hline $2: 41: 15$ & 31.9 & 10.8 & 51.3 & 20.1 & 28.9 & 27.9 & 17.6 \\
\hline $2: 41: 30$ & 31.9 & 10.8 & 51.4 & 20.1 & 28.9 & 27.9 & 17.3 \\
\hline $2: 41: 45$ & 31.9 & 10.8 & 51.5 & 20.1 & 28.9 & 28 & 17.5 \\
\hline $2: 42: 00$ & 31.9 & 10.8 & 51.5 & 20.1 & 28.8 & 27.9 & 17.5 \\
\hline $2: 42: 15$ & 32 & 10.8 & 51.5 & 20.1 & 29 & 28 & 17.4 \\
\hline $2: 42: 30$ & 32 & 10.9 & 51.5 & 20.1 & 28.8 & 28 & 17.5 \\
\hline $2: 42: 45$ & 31.9 & 10.9 & 51.5 & 20.1 & 28.8 & 27.9 & 17.5 \\
\hline $2: 43: 00$ & 31.9 & 10.9 & 51.5 & 20.1 & 28.8 & 27.9 & 17.6 \\
\hline $2: 43: 15$ & 31.9 & 11 & 51.5 & 20.1 & 28.8 & 27.9 & 17.5 \\
\hline $2: 43: 30$ & 31.9 & 10.9 & 51.5 & 20.1 & 28.9 & 28 & 17.5 \\
\hline $2: 43: 45$ & 31.9 & 11 & 51.5 & 20.1 & 28.8 & 27.9 & 17.5 \\
\hline $2: 44: 00$ & 31.9 & 11 & 51.6 & 20.1 & 28.8 & 27.9 & 17.5 \\
\hline $2: 44: 15$ & 31.9 & 11 & 51.6 & 20.1 & 28.8 & 27.9 & 17.5 \\
\hline $2: 44: 30$ & 31.9 & 11 & 51.6 & 20.1 & 28.9 & 28 & 17.6 \\
\hline $2: 44: 45$ & 32 & 11 & 51.6 & 20.1 & 28.8 & 27.9 & 17.5 \\
\hline $2: 45: 00$ & 31.9 & 11.1 & 51.6 & 20.1 & 28.8 & 27.9 & 17.5 \\
\hline $2: 45: 15$ & 31.9 & 11.1 & 51.6 & 20.1 & 28.8 & 27.9 & 17.5 \\
\hline $2: 45: 30$ & 31.9 & 11.1 & 51.6 & 20.1 & 28.8 & 27.9 & 17.6 \\
\hline $2: 45: 45$ & 31.9 & 11.1 & 51.6 & 20.2 & 28.8 & 27.9 & 17.5 \\
\hline $2: 46: 00$ & 31.9 & 11.1 & 51.6 & 20.1 & 28.8 & 27.9 & 17.6 \\
\hline $2: 46: 15$ & 31.9 & 11.1 & 51.6 & 20.1 & 28.8 & 27.9 & 17.7 \\
\hline $2: 46: 30$ & 31.9 & 11.2 & 51.6 & 20.1 & 28.8 & 27.9 & 17.6 \\
\hline $2: 46: 45$ & 31.9 & 11.2 & 51.6 & 20.2 & 28.8 & 27.9 & 17.6 \\
\hline $2: 47: 00$ & 31.9 & 11.2 & 51.6 & 20.1 & 28.8 & 27.9 & 17.4 \\
\hline $2: 47: 15$ & 31.9 & 11.2 & 51.6 & 20.2 & 28.8 & 27.9 & 17.6 \\
\hline $2: 47: 30$ & 31.9 & 11.2 & 51.6 & 20.1 & 28.8 & 27.9 & 17.6 \\
\hline $2: 47: 45$ & 31.9 & 11.2 & 51.7 & 20.2 & 28.8 & 27.9 & 17.5 \\
\hline $2: 48: 00$ & 31.9 & 11.2 & 51.7 & 20.2 & 28.8 & 27.9 & 17.6 \\
\hline $2: 48: 15$ & 31.9 & 11.2 & 51.7 & 20.2 & 28.8 & 27.9 & 17.7 \\
\hline $2: 48: 30$ & 31.9 & 11.2 & 51.7 & 20.2 & 28.8 & 27.9 & 17.4 \\
\hline $2: 48: 45$ & 31.9 & 11.2 & 51.7 & 20.2 & 28.8 & 27.9 & 17.6 \\
\hline $2: 49: 00$ & 31.8 & 11.3 & 51.6 & 20.2 & 28.8 & 27.8 & 17.6 \\
\hline $2: 49: 15$ & 31.8 & 11.3 & 51.7 & 20.2 & 28.8 & 27.9 & 17.6 \\
\hline $2: 49: 30$ & 31.8 & 11.3 & 51.7 & 20.2 & 28.8 & 27.9 & 17.6 \\
\hline $2: 49: 45$ & 31.8 & 11.3 & 51.6 & 20.2 & 28.8 & 27.9 & 17.6 \\
\hline $2: 50: 00$ & 31.9 & 11.3 & 51.6 & 20.2 & 28.8 & 27.9 & 17.6 \\
\hline $2: 50: 15$ & 31.8 & 11.2 & 51.6 & 20.2 & 28.9 & 28 & 17.6 \\
\hline $2: 50: 30$ & 31.8 & 11.3 & 51.6 & 20.2 & 28.8 & 27.9 & 17.5 \\
\hline $2: 50: 45$ & 31.8 & 11.3 & 51.6 & 20.2 & 28.8 & 27.9 & 17.6 \\
\hline
\end{tabular}




\begin{tabular}{|c|c|c|c|c|c|c|c|}
\hline $2: 51: 00$ & 31.8 & 11.3 & 51.7 & 20.2 & 28.8 & 27.9 & 17.7 \\
\hline $2: 51: 15$ & 31.8 & 11.3 & 51.7 & 20.2 & 28.8 & 27.9 & 17.6 \\
\hline $2: 51: 30$ & 31.8 & 11.3 & 51.7 & 20.2 & 28.8 & 27.9 & 17.6 \\
\hline $2: 51: 45$ & 31.8 & 11.3 & 51.6 & 20.2 & 28.8 & 27.9 & 17.6 \\
\hline $2: 52: 00$ & 31.8 & 11.3 & 51.7 & 20.2 & 28.8 & 27.9 & 17.7 \\
\hline $2: 52: 15$ & 31.8 & 11.3 & 51.7 & 20.2 & 28.8 & 27.8 & 17.6 \\
\hline $2: 52: 30$ & 31.8 & 11.3 & 51.7 & 20.2 & 28.8 & 27.9 & 17.5 \\
\hline $2: 52: 45$ & 31.8 & 11.3 & 51.7 & 20.2 & 28.8 & 27.9 & 17.7 \\
\hline $2: 53: 00$ & 31.8 & 11.3 & 51.7 & 20.2 & 28.8 & 27.9 & 17.5 \\
\hline $2: 53: 15$ & 31.8 & 11.3 & 51.7 & 20.2 & 28.8 & 27.9 & 17.5 \\
\hline $2: 53: 30$ & 31.8 & 11.3 & 51.7 & 20.2 & 28.8 & 27.8 & 17.8 \\
\hline $2: 53: 45$ & 31.8 & 11.3 & 51.7 & 20.2 & 28.8 & 27.9 & 17.6 \\
\hline $2: 54: 00$ & 31.8 & 11.3 & 51.7 & 20.2 & 28.8 & 27.9 & 17.7 \\
\hline $2: 54: 15$ & 31.8 & 11.3 & 51.7 & 20.2 & 28.8 & 27.9 & 17.5 \\
\hline $2: 54: 30$ & 31.7 & 11.3 & 51.7 & 20.2 & 28.8 & 27.9 & 17.6 \\
\hline $2: 54: 45$ & 31.8 & 11.3 & 51.7 & 20.2 & 28.8 & 27.9 & 17.7 \\
\hline $2: 55: 00$ & 31.8 & 11.3 & 51.7 & 20.2 & 28.8 & 27.8 & 17.5 \\
\hline $2: 55: 15$ & 31.8 & 11.3 & 51.7 & 20.2 & 28.8 & 27.8 & 17.7 \\
\hline $2: 55: 30$ & 31.8 & 11.3 & 51.7 & 20.2 & 28.8 & 27.9 & 17.6 \\
\hline $2: 55: 45$ & 31.8 & 11.3 & 51.8 & 20.2 & 28.8 & 27.9 & 17.7 \\
\hline $2: 56: 00$ & 31.8 & 11.3 & 51.7 & 20.2 & 28.8 & 27.9 & 17.7 \\
\hline $2: 56: 15$ & 31.8 & 11.3 & 51.7 & 20.2 & 28.8 & 27.9 & 17.8 \\
\hline $2: 56: 30$ & 31.8 & 11.3 & 51.8 & 20.3 & 28.8 & 27.8 & 17.6 \\
\hline $2: 56: 45$ & 31.8 & 11.3 & 51.7 & 20.3 & 28.8 & 27.8 & 17.8 \\
\hline $2: 57: 00$ & 31.8 & 11.3 & 51.8 & 20.3 & 28.8 & 27.9 & 17.6 \\
\hline $2: 57: 15$ & 31.8 & 11.3 & 51.8 & 20.3 & 28.8 & 27.9 & 17.5 \\
\hline $2: 57: 30$ & 31.8 & 11.3 & 51.8 & 20.3 & 28.8 & 27.8 & 17.7 \\
\hline $2: 57: 45$ & 31.8 & 11.3 & 51.8 & 20.3 & 28.8 & 27.9 & 17.7 \\
\hline $2: 58: 00$ & 31.8 & 11.3 & 51.8 & 20.3 & 28.8 & 27.9 & 17.6 \\
\hline $2: 58: 15$ & 31.8 & 11.3 & 51.8 & 20.3 & 28.8 & 27.8 & 17.8 \\
\hline $2: 58: 30$ & 31.8 & 11.3 & 51.8 & 20.3 & 28.8 & 27.9 & 17.7 \\
\hline $2: 58: 45$ & 31.8 & 11.3 & 51.8 & 20.3 & 28.8 & 27.9 & 17.8 \\
\hline $2: 59: 00$ & 31.8 & 11.3 & 51.8 & 20.3 & 28.7 & 27.8 & 17.8 \\
\hline $2: 59: 15$ & 31.7 & 11.3 & 51.8 & 20.3 & 28.8 & 27.9 & 17.7 \\
\hline $2: 59: 30$ & 31.7 & 11.3 & 51.8 & 20.3 & 28.8 & 27.9 & 17.7 \\
\hline $2: 59: 45$ & 31.8 & 11.3 & 51.8 & 20.3 & 28.8 & 27.8 & 17.9 \\
\hline $3: 00: 00$ & 31.8 & 11.3 & 51.8 & 20.3 & 28.8 & 27.9 & 17.9 \\
\hline $3: 00: 15$ & 31.8 & 11.3 & 51.8 & 20.3 & 28.7 & 27.9 & 17.8 \\
\hline $3: 00: 30$ & 31.8 & 11.3 & 51.8 & 20.3 & 28.8 & 27.8 & 17.8 \\
\hline $3: 00: 45$ & 31.8 & 11.3 & 51.8 & 20.3 & 28.8 & 27.9 & 17.8 \\
\hline $3: 01: 00$ & 31.8 & 11.3 & 51.8 & 20.3 & 28.8 & 27.9 & 18 \\
\hline $3: 01: 15$ & 31.8 & 11.3 & 51.8 & 20.3 & 28.8 & 27.8 & 18 \\
\hline $3: 01: 30$ & 31.8 & 11.3 & 51.9 & 20.3 & 28.8 & 27.8 & 17.9 \\
\hline
\end{tabular}




\begin{tabular}{|c|c|c|c|c|c|c|c|}
\hline $3: 01: 45$ & 31.8 & 11.3 & 51.8 & 20.3 & 28.7 & 27.9 & 17.9 \\
\hline $3: 02: 00$ & 31.8 & 11.3 & 51.8 & 20.3 & 28.8 & 27.8 & 17.9 \\
\hline $3: 02: 15$ & 31.8 & 11.3 & 51.8 & 20.3 & 28.8 & 27.8 & 17.7 \\
\hline $3: 02: 30$ & 31.8 & 11.3 & 51.8 & 20.3 & 28.8 & 27.9 & 17.7 \\
\hline $3: 02: 45$ & 31.8 & 11.3 & 51.8 & 20.3 & 28.8 & 27.9 & 17.7 \\
\hline $3: 03: 00$ & 31.8 & 11.3 & 51.9 & 20.3 & 28.8 & 27.9 & 17.9 \\
\hline $3: 03: 15$ & 31.8 & 11.3 & 51.9 & 20.3 & 28.8 & 27.9 & 17.6 \\
\hline $3: 03: 30$ & 31.8 & 11.3 & 51.8 & 20.3 & 28.7 & 27.8 & 17.7 \\
\hline $3: 03: 45$ & 31.8 & 11.3 & 51.8 & 20.3 & 28.7 & 27.8 & 17.7 \\
\hline $3: 04: 00$ & 31.8 & 11.3 & 51.8 & 20.3 & 28.8 & 27.9 & 17.6 \\
\hline $3: 04: 15$ & 31.8 & 11.3 & 51.8 & 20.3 & 28.8 & 27.8 & 17.4 \\
\hline $3: 04: 30$ & 31.8 & 11.3 & 51.9 & 20.3 & 28.8 & 27.8 & 17.5 \\
\hline $3: 04: 45$ & 31.8 & 11.3 & 51.9 & 20.3 & 28.8 & 27.9 & 17.4 \\
\hline $3: 05: 00$ & 31.8 & 11.3 & 51.9 & 20.3 & 28.7 & 27.8 & 17.2 \\
\hline $3: 05: 15$ & 31.8 & 11.3 & 51.9 & 20.3 & 28.7 & 27.8 & 17.1 \\
\hline $3: 05: 30$ & 31.8 & 11.3 & 51.9 & 20.3 & 28.7 & 27.9 & 17.2 \\
\hline $3: 05: 45$ & 31.8 & 11.3 & 51.9 & 20.3 & 28.8 & 27.8 & 16.7 \\
\hline 3:06:00 & 31.8 & 11.3 & 51.9 & 20.3 & 28.8 & 27.9 & 17 \\
\hline $3: 06: 15$ & 31.8 & 11.3 & 51.9 & 20.3 & 28.8 & 27.9 & 16.7 \\
\hline $3: 06: 30$ & 31.7 & 11.3 & 51.9 & 20.3 & 28.8 & 27.9 & 16.6 \\
\hline $3: 06: 45$ & 31.7 & 11.4 & 51.8 & 20.3 & 28.7 & 27.8 & 16.6 \\
\hline $3: 07: 00$ & 31.7 & 11.3 & 51.9 & 20.3 & 28.8 & 27.9 & 16.4 \\
\hline $3: 07: 15$ & 31.7 & 11.3 & 51.9 & 20.3 & 28.8 & 27.8 & 16.4 \\
\hline $3: 07: 30$ & 31.7 & 11.3 & 51.9 & 20.3 & 28.7 & 27.8 & 16.2 \\
\hline $3: 07: 45$ & 31.7 & 11.4 & 51.9 & 20.3 & 28.8 & 27.8 & 16.2 \\
\hline $3: 08: 00$ & 31.7 & 11.4 & 51.9 & 20.3 & 28.8 & 27.9 & 16.2 \\
\hline $3: 08: 15$ & 31.7 & 11.4 & 51.9 & 20.3 & 28.7 & 27.8 & 16.1 \\
\hline $3: 08: 30$ & 31.7 & 11.4 & 51.9 & 20.3 & 28.7 & 27.8 & 16 \\
\hline $3: 08: 45$ & 31.7 & 11.4 & 51.9 & 20.3 & 28.7 & 27.8 & 16 \\
\hline $3: 09: 00$ & 31.7 & 11.4 & 51.9 & 20.3 & 28.7 & 27.8 & 16.1 \\
\hline $3: 09: 15$ & 31.7 & 11.3 & 51.9 & 20.3 & 28.7 & 27.8 & 16.1 \\
\hline $3: 09: 30$ & 31.7 & 11.3 & 51.9 & 20.3 & 28.7 & 27.8 & 16.1 \\
\hline $3: 09: 45$ & 31.7 & 11.3 & 51.9 & 20.3 & 28.7 & 27.8 & 16.2 \\
\hline $3: 10: 00$ & 31.7 & 11.3 & 51.9 & 20.2 & 28.8 & 27.8 & 16 \\
\hline $3: 10: 15$ & 31.7 & 11.3 & 51.9 & 20.2 & 28.7 & 27.8 & 16.2 \\
\hline $3: 10: 30$ & 31.6 & 11.3 & 51.9 & 20.2 & 28.7 & 27.8 & 16.2 \\
\hline $3: 10: 45$ & 31.6 & 11.3 & 51.8 & 20.2 & 28.7 & 27.8 & 16.1 \\
\hline $3: 11: 00$ & 31.6 & 11.3 & 51.9 & 20.3 & 28.8 & 27.9 & 16.1 \\
\hline $3: 11: 15$ & 31.7 & 11.3 & 51.9 & 20.3 & 28.7 & 27.8 & 16.1 \\
\hline $3: 11: 30$ & 31.6 & 11.2 & 51.9 & 20.2 & 28.7 & 27.8 & 16.3 \\
\hline $3: 11: 45$ & 31.7 & 11.3 & 51.9 & 20.2 & 28.7 & 27.8 & 16.2 \\
\hline $3: 12: 00$ & 31.7 & 11.2 & 51.9 & 20.3 & 28.7 & 27.8 & 16.2 \\
\hline $3: 12: 15$ & 31.7 & 11.2 & 51.9 & 20.3 & 28.7 & 27.8 & 16.2 \\
\hline
\end{tabular}




\begin{tabular}{|c|c|c|c|c|c|c|c|}
\hline $3: 12: 30$ & 31.6 & 11.2 & 51.9 & 20.2 & 28.8 & 27.8 & 16.1 \\
\hline $3: 12: 45$ & 31.6 & 11.2 & 51.9 & 20.2 & 28.7 & 27.8 & 16.3 \\
\hline $3: 13: 00$ & 31.6 & 11.2 & 51.8 & 20.2 & 28.7 & 27.8 & 16.4 \\
\hline $3: 13: 15$ & 31.7 & 11.2 & 51.8 & 20.2 & 28.7 & 27.8 & 16.3 \\
\hline $3: 13: 30$ & 31.6 & 11.2 & 51.8 & 20.2 & 28.7 & 27.8 & 16.3 \\
\hline $3: 13: 45$ & 31.6 & 11.2 & 51.8 & 20.2 & 28.7 & 27.8 & 16.3 \\
\hline $3: 14: 00$ & 31.7 & 11.2 & 51.8 & 20.2 & 28.7 & 27.8 & 16.4 \\
\hline $3: 14: 15$ & 31.7 & 11.1 & 51.8 & 20.2 & 28.7 & 27.8 & 16.4 \\
\hline $3: 14: 30$ & 31.7 & 11.2 & 51.8 & 20.2 & 28.7 & 27.8 & 16.4 \\
\hline $3: 14: 45$ & 31.7 & 11.1 & 51.8 & 20.2 & 28.7 & 27.8 & 16.4 \\
\hline $3: 15: 00$ & 31.6 & 11.1 & 51.8 & 20.2 & 28.7 & 27.8 & 16.5 \\
\hline $3: 15: 15$ & 31.6 & 11.1 & 51.8 & 20.2 & 28.7 & 27.8 & 16.3 \\
\hline $3: 15: 30$ & 31.6 & 11.1 & 51.8 & 20.2 & 28.7 & 27.8 & 16.2 \\
\hline $3: 15: 45$ & 31.6 & 11.1 & 51.8 & 20.2 & 28.7 & 27.8 & 16.5 \\
\hline $3: 16: 00$ & 31.6 & 11.1 & 51.8 & 20.2 & 28.7 & 27.8 & 16.5 \\
\hline $3: 16: 15$ & 31.6 & 11.1 & 51.8 & 20.2 & 28.7 & 27.8 & 16.5 \\
\hline $3: 16: 30$ & 31.6 & 11.1 & 51.8 & 20.2 & 28.7 & 27.8 & 16.5 \\
\hline $3: 16: 45$ & 31.7 & 11.1 & 51.8 & 20.2 & 28.7 & 27.8 & 16.5 \\
\hline $3: 17: 00$ & 31.7 & 11.1 & 51.8 & 20.2 & 28.7 & 27.9 & 16.5 \\
\hline $3: 17: 15$ & 31.6 & 11.1 & 51.8 & 20.2 & 28.7 & 27.8 & 16.4 \\
\hline $3: 17: 30$ & 31.7 & 11 & 51.8 & 20.2 & 28.7 & 27.8 & 16.4 \\
\hline $3: 17: 45$ & 31.7 & 11 & 51.8 & 20.2 & 28.7 & 27.8 & 16.4 \\
\hline $3: 18: 00$ & 31.7 & 11.1 & 51.8 & 20.2 & 28.7 & 27.8 & 16.6 \\
\hline $3: 18: 15$ & 31.7 & 11 & 51.8 & 20.2 & 28.7 & 27.8 & 16.5 \\
\hline $3: 18: 30$ & 31.7 & 11 & 51.8 & 20.2 & 28.7 & 27.8 & 16.7 \\
\hline $3: 18: 45$ & 31.6 & 11.1 & 51.8 & 20.2 & 28.8 & 27.8 & 16.5 \\
\hline $3: 19: 00$ & 31.6 & 11.1 & 51.8 & 20.2 & 28.7 & 27.8 & 16.6 \\
\hline $3: 19: 15$ & 31.6 & 11.1 & 51.8 & 20.2 & 28.7 & 27.8 & 16.7 \\
\hline $3: 19: 30$ & 31.6 & 11.1 & 51.8 & 20.2 & 28.7 & 27.8 & 16.5 \\
\hline $3: 19: 45$ & 31.6 & 11.1 & 51.8 & 20.2 & 28.7 & 27.8 & 16.6 \\
\hline $3: 20: 00$ & 31.6 & 11.1 & 51.8 & 20.2 & 28.7 & 27.8 & 16.7 \\
\hline $3: 20: 15$ & 31.7 & 11 & 51.8 & 20.2 & 28.7 & 27.8 & 16.6 \\
\hline $3: 20: 30$ & 31.7 & 11 & 51.8 & 20.2 & 28.8 & 27.8 & 16.7 \\
\hline $3: 20: 45$ & 31.7 & 11.1 & 51.8 & 20.2 & 28.7 & 27.9 & 16.5 \\
\hline $3: 21: 00$ & 31.7 & 11 & 51.8 & 20.2 & 28.8 & 27.8 & 16.8 \\
\hline $3: 21: 15$ & 31.7 & 11.1 & 51.8 & 20.2 & 28.7 & 27.8 & 16.7 \\
\hline $3: 21: 30$ & 31.7 & 11.1 & 51.8 & 20.2 & 28.7 & 27.8 & 16.7 \\
\hline $3: 21: 45$ & 31.7 & 11.1 & 51.8 & 20.2 & 28.7 & 27.8 & 16.7 \\
\hline $3: 22: 00$ & 31.7 & 11.1 & 51.8 & 20.2 & 28.7 & 27.8 & 16.6 \\
\hline $3: 22: 15$ & 31.7 & 11.1 & 51.8 & 20.2 & 28.7 & 27.8 & 16.8 \\
\hline $3: 22: 30$ & 31.6 & 11 & 51.8 & 20.2 & 28.8 & 27.9 & 16.7 \\
\hline $3: 22: 45$ & 31.7 & 11 & 51.8 & 20.2 & 28.7 & 27.8 & 16.7 \\
\hline $3: 23: 00$ & 31.7 & 11 & 51.8 & 20.2 & 28.7 & 27.8 & 16.9 \\
\hline
\end{tabular}




\begin{tabular}{|c|c|c|c|c|c|c|c|}
\hline $3: 23: 15$ & 31.7 & 11 & 51.8 & 20.2 & 28.7 & 27.8 & 16.7 \\
\hline $3: 23: 30$ & 31.7 & 11 & 51.8 & 20.2 & 28.7 & 27.8 & 16.8 \\
\hline $3: 23: 45$ & 31.7 & 11 & 51.8 & 20.2 & 28.7 & 27.9 & 16.8 \\
\hline $3: 24: 00$ & 31.7 & 11 & 51.8 & 20.2 & 28.7 & 27.8 & 16.8 \\
\hline $3: 24: 15$ & 31.7 & 11 & 51.8 & 20.2 & 28.7 & 27.8 & 16.9 \\
\hline $3: 24: 30$ & 31.7 & 11 & 51.8 & 20.2 & 28.8 & 27.9 & 16.9 \\
\hline $3: 24: 45$ & 31.7 & 11 & 51.8 & 20.2 & 28.8 & 27.9 & 16.9 \\
\hline $3: 25: 00$ & 31.7 & 11 & 51.8 & 20.2 & 28.7 & 27.8 & 16.9 \\
\hline $3: 25: 15$ & 31.6 & 11 & 51.8 & 20.2 & 28.7 & 27.8 & 17.1 \\
\hline $3: 25: 30$ & 31.7 & 11 & 51.8 & 20.2 & 28.7 & 27.8 & 17 \\
\hline $3: 25: 45$ & 31.6 & 11 & 51.8 & 20.2 & 28.7 & 27.8 & 16.9 \\
\hline $3: 26: 00$ & 31.7 & 11 & 51.8 & 20.2 & 28.8 & 27.9 & 17 \\
\hline $3: 26: 15$ & 31.6 & 11 & 51.7 & 20.2 & 28.7 & 27.8 & 17.1 \\
\hline $3: 26: 30$ & 31.7 & 11 & 51.8 & 20.2 & 28.7 & 27.8 & 17.1 \\
\hline $3: 26: 45$ & 31.7 & 11 & 51.7 & 20.2 & 28.8 & 27.9 & 17.2 \\
\hline $3: 27: 00$ & 31.7 & 11 & 51.8 & 20.2 & 28.8 & 27.9 & 17.1 \\
\hline $3: 27: 15$ & 31.6 & 11 & 51.8 & 20.2 & 28.7 & 27.8 & 17.2 \\
\hline $3: 27: 30$ & 31.6 & 11 & 51.8 & 20.2 & 28.7 & 27.8 & 17 \\
\hline $3: 27: 45$ & 31.6 & 11 & 51.8 & 20.2 & 28.7 & 27.9 & 17.2 \\
\hline $3: 28: 00$ & 31.6 & 11 & 51.7 & 20.2 & 28.8 & 27.9 & 17.2 \\
\hline $3: 28: 15$ & 31.7 & 11 & 51.8 & 20.2 & 28.8 & 27.9 & 17.2 \\
\hline $3: 28: 30$ & 31.7 & 11 & 51.8 & 20.2 & 28.8 & 27.9 & 17.4 \\
\hline $3: 28: 45$ & 31.7 & 11 & 51.8 & 20.2 & 28.8 & 27.8 & 17.2 \\
\hline $3: 29: 00$ & 31.7 & 11 & 51.7 & 20.2 & 28.7 & 27.8 & 17.3 \\
\hline $3: 29: 15$ & 31.7 & 11 & 51.8 & 20.2 & 28.8 & 27.9 & 17.1 \\
\hline $3: 29: 30$ & 31.6 & 11 & 51.7 & 20.2 & 28.8 & 27.8 & 17.3 \\
\hline $3: 29: 45$ & 31.7 & 11.1 & 51.8 & 20.2 & 28.8 & 27.8 & 17.2 \\
\hline $3: 30: 00$ & 31.7 & 11 & 51.8 & 20.2 & 28.8 & 27.9 & 17.3 \\
\hline $3: 30: 15$ & 31.7 & 11 & 51.8 & 20.2 & 28.7 & 27.8 & 17.2 \\
\hline $3: 30: 30$ & 31.6 & 11 & 51.8 & 20.2 & 28.8 & 27.9 & 17.3 \\
\hline $3: 30: 45$ & 31.6 & 11 & 51.8 & 20.2 & 28.8 & 27.9 & 17.2 \\
\hline $3: 31: 00$ & 31.7 & 11 & 51.8 & 20.2 & 28.7 & 27.8 & 17.1 \\
\hline $3: 31: 15$ & 31.6 & 11 & 51.8 & 20.2 & 28.8 & 27.8 & 17.3 \\
\hline $3: 31: 30$ & 31.7 & 11 & 51.8 & 20.2 & 28.7 & 27.8 & 17.2 \\
\hline $3: 31: 45$ & 31.7 & 11 & 51.8 & 20.2 & 28.7 & 27.8 & 17 \\
\hline $3: 32: 00$ & 31.7 & 11.1 & 51.8 & 20.2 & 28.7 & 27.8 & 17.1 \\
\hline $3: 32: 15$ & 31.7 & 11 & 51.8 & 20.2 & 28.8 & 27.9 & 17.1 \\
\hline $3: 32: 30$ & 31.7 & 11 & 51.8 & 20.2 & 28.8 & 27.8 & 16.9 \\
\hline $3: 32: 45$ & 31.7 & 11 & 51.8 & 20.2 & 28.8 & 27.9 & 17 \\
\hline $3: 33: 00$ & 31.7 & 11 & 51.8 & 20.2 & 28.7 & 27.9 & 17 \\
\hline $3: 33: 15$ & 31.6 & 11 & 51.8 & 20.2 & 28.8 & 27.9 & 16.9 \\
\hline $3: 33: 30$ & 31.6 & 11 & 51.8 & 20.2 & 28.8 & 27.8 & 16.7 \\
\hline $3: 33: 45$ & 31.6 & 11 & 51.7 & 20.2 & 28.7 & 27.9 & 16.9 \\
\hline
\end{tabular}




\begin{tabular}{|c|c|c|c|c|c|c|c|}
\hline $3: 34: 00$ & 31.6 & 11 & 51.8 & 20.2 & 28.8 & 27.9 & 16.9 \\
\hline $3: 34: 15$ & 31.7 & 11 & 51.8 & 20.2 & 28.8 & 27.8 & 16.8 \\
\hline $3: 34: 30$ & 31.7 & 10.9 & 51.8 & 20.2 & 28.8 & 27.9 & 16.9 \\
\hline $3: 34: 45$ & 31.6 & 10.9 & 51.7 & 20.2 & 28.8 & 27.9 & 16.9 \\
\hline $3: 35: 00$ & 31.7 & 10.9 & 51.8 & 20.2 & 28.8 & 27.8 & 17 \\
\hline $3: 35: 15$ & 31.7 & 10.9 & 51.8 & 20.2 & 28.8 & 27.9 & 16.9 \\
\hline $3: 35: 30$ & 31.7 & 10.9 & 51.8 & 20.2 & 28.8 & 27.9 & 16.8 \\
\hline $3: 35: 45$ & 31.6 & 10.9 & 51.8 & 20.2 & 28.8 & 27.8 & 16.9 \\
\hline $3: 36: 00$ & 31.6 & 10.9 & 51.8 & 20.2 & 28.8 & 27.9 & 16.9 \\
\hline $3: 36: 15$ & 31.6 & 10.9 & 51.8 & 20.2 & 28.8 & 27.9 & 16.9 \\
\hline $3: 36: 30$ & 31.6 & 10.9 & 51.8 & 20.2 & 28.8 & 27.8 & 16.9 \\
\hline $3: 36: 45$ & 31.6 & 10.9 & 51.8 & 20.2 & 28.7 & 27.9 & 16.9 \\
\hline $3: 37: 00$ & 31.6 & 10.9 & 51.8 & 20.2 & 28.7 & 27.8 & 16.8 \\
\hline $3: 37: 15$ & 31.7 & 10.9 & 51.7 & 20.2 & 28.8 & 27.8 & 17 \\
\hline $3: 37: 30$ & 31.7 & 10.8 & 51.8 & 20.2 & 28.8 & 27.9 & 16.8 \\
\hline $3: 37: 45$ & 31.7 & 10.9 & 51.8 & 20.2 & 28.7 & 27.8 & 16.9 \\
\hline $3: 38: 00$ & 31.7 & 10.8 & 51.8 & 20.2 & 28.8 & 27.9 & 16.8 \\
\hline $3: 38: 15$ & 31.7 & 10.8 & 51.8 & 20.2 & 28.8 & 27.9 & 16.9 \\
\hline $3: 38: 30$ & 31.7 & 10.8 & 51.8 & 20.2 & 28.8 & 27.9 & 16.9 \\
\hline $3: 38: 45$ & 31.7 & 10.9 & 51.7 & 20.2 & 28.7 & 27.8 & 16.9 \\
\hline $3: 39: 00$ & 31.7 & 10.8 & 51.8 & 20.2 & 28.8 & 27.9 & 16.9 \\
\hline $3: 39: 15$ & 31.7 & 10.8 & 51.8 & 20.2 & 28.8 & 27.9 & 16.9 \\
\hline $3: 39: 30$ & 31.7 & 10.8 & 51.8 & 20.2 & 28.8 & 27.8 & 16.9 \\
\hline $3: 39: 45$ & 31.7 & 10.8 & 51.8 & 20.2 & 28.8 & 27.8 & 16.9 \\
\hline $3: 40: 00$ & 31.7 & 10.8 & 51.8 & 20.2 & 28.8 & 27.9 & 17.1 \\
\hline $3: 40: 15$ & 31.7 & 10.8 & 51.8 & 20.2 & 28.8 & 27.8 & 16.9 \\
\hline $3: 40: 30$ & 31.7 & 10.8 & 51.8 & 20.2 & 28.8 & 27.9 & 16.9 \\
\hline $3: 40: 45$ & 31.7 & 10.8 & 51.8 & 20.2 & 28.8 & 27.9 & 17 \\
\hline $3: 41: 00$ & 31.7 & 10.8 & 51.8 & 20.2 & 28.8 & 27.9 & 17 \\
\hline $3: 41: 15$ & 31.7 & 10.8 & 51.8 & 20.2 & 28.8 & 27.9 & 16.9 \\
\hline $3: 41: 30$ & 31.7 & 10.8 & 51.8 & 20.2 & 28.8 & 27.9 & 17.1 \\
\hline $3: 41: 45$ & 31.7 & 10.8 & 51.8 & 20.2 & 28.8 & 27.8 & 17 \\
\hline $3: 42: 00$ & 31.7 & 10.8 & 51.8 & 20.2 & 28.8 & 27.9 & 17 \\
\hline $3: 42: 15$ & 31.7 & 10.7 & 51.8 & 20.2 & 28.8 & 27.9 & 17 \\
\hline $3: 42: 30$ & 31.7 & 10.8 & 51.8 & 20.2 & 28.8 & 27.8 & 17.1 \\
\hline $3: 42: 45$ & 31.7 & 10.8 & 51.8 & 20.2 & 28.8 & 27.9 & 17 \\
\hline $3: 43: 00$ & 31.7 & 10.9 & 51.8 & 20.2 & 28.8 & 27.9 & 17.2 \\
\hline $3: 43: 15$ & 31.7 & 10.9 & 51.8 & 20.2 & 28.8 & 27.9 & 17.2 \\
\hline $3: 43: 30$ & 31.7 & 10.8 & 51.8 & 20.2 & 28.8 & 27.9 & 17.1 \\
\hline $3: 43: 45$ & 31.7 & 10.8 & 51.8 & 20.2 & 28.8 & 27.9 & 17 \\
\hline $3: 44: 00$ & 31.6 & 10.8 & 51.8 & 20.2 & 28.8 & 27.9 & 16.9 \\
\hline $3: 44: 15$ & 31.7 & 10.8 & 51.8 & 20.2 & 28.8 & 27.8 & 16.9 \\
\hline $3: 44: 30$ & 31.6 & 10.8 & 51.8 & 20.2 & 28.8 & 27.9 & 16.9 \\
\hline
\end{tabular}




\begin{tabular}{|c|c|c|c|c|c|c|c|}
\hline $3: 44: 45$ & 31.7 & 10.8 & 51.8 & 20.2 & 28.7 & 27.8 & 17 \\
\hline $3: 45: 00$ & 31.7 & 10.8 & 51.8 & 20.2 & 28.8 & 27.9 & 17 \\
\hline $3: 45: 15$ & 31.7 & 10.7 & 51.8 & 20.1 & 28.9 & 28 & 17 \\
\hline $3: 45: 30$ & 31.7 & 10.8 & 51.8 & 20.1 & 28.8 & 27.9 & 16.9 \\
\hline $3: 45: 45$ & 31.7 & 10.8 & 51.8 & 20.1 & 28.8 & 27.8 & 17.1 \\
\hline $3: 46: 00$ & 31.7 & 10.8 & 51.8 & 20.1 & 28.8 & 27.9 & 17.1 \\
\hline $3: 46: 15$ & 31.7 & 10.8 & 51.7 & 20.1 & 28.8 & 27.9 & 17.1 \\
\hline $3: 46: 30$ & 31.7 & 10.8 & 51.7 & 20.1 & 28.8 & 27.8 & 17.1 \\
\hline $3: 46: 45$ & 31.7 & 10.7 & 51.7 & 20.1 & 28.8 & 27.9 & 17.1 \\
\hline $3: 47: 00$ & 31.7 & 10.7 & 51.7 & 20.1 & 28.8 & 27.8 & 17.3 \\
\hline $3: 47: 15$ & 31.7 & 10.7 & 51.7 & 20.1 & 28.8 & 27.8 & 17.1 \\
\hline $3: 47: 30$ & 31.7 & 10.7 & 51.7 & 20.1 & 28.8 & 27.9 & 17 \\
\hline $3: 47: 45$ & 31.7 & 10.7 & 51.7 & 20.1 & 28.8 & 27.9 & 17.1 \\
\hline $3: 48: 00$ & 31.7 & 10.7 & 51.7 & 20.1 & 28.8 & 27.8 & 17.1 \\
\hline $3: 48: 15$ & 31.7 & 10.6 & 51.7 & 20.1 & 28.8 & 27.9 & 17.1 \\
\hline $3: 48: 30$ & 31.7 & 10.6 & 51.7 & 20.1 & 28.8 & 27.9 & 17.2 \\
\hline $3: 48: 45$ & 31.7 & 10.6 & 51.7 & 20.1 & 28.8 & 27.8 & 17.1 \\
\hline $3: 49: 00$ & 31.7 & 10.6 & 51.7 & 20.1 & 28.8 & 27.9 & 17.2 \\
\hline $3: 49: 15$ & 31.7 & 10.6 & 51.7 & 20.1 & 28.8 & 27.9 & 17.2 \\
\hline $3: 49: 30$ & 31.7 & 10.6 & 51.7 & 20.1 & 28.8 & 27.8 & 17 \\
\hline $3: 49: 45$ & 31.7 & 10.6 & 51.8 & 20.1 & 28.8 & 27.9 & 17.2 \\
\hline $3: 50: 00$ & 31.7 & 10.6 & 51.7 & 20.1 & 28.8 & 27.9 & 17.2 \\
\hline $3: 50: 15$ & 31.7 & 10.7 & 51.7 & 20.1 & 28.8 & 27.9 & 17.1 \\
\hline $3: 50: 30$ & 31.7 & 10.7 & 51.7 & 20.1 & 28.8 & 27.8 & 17.1 \\
\hline $3: 50: 45$ & 31.7 & 10.7 & 51.8 & 20.1 & 28.8 & 27.9 & 17.2 \\
\hline $3: 51: 00$ & 31.7 & 10.7 & 51.8 & 20.1 & 28.8 & 27.9 & 17.2 \\
\hline $3: 51: 15$ & 31.7 & 10.7 & 51.8 & 20.1 & 28.9 & 27.9 & 17.3 \\
\hline $3: 51: 30$ & 31.7 & 10.7 & 51.8 & 20.1 & 28.8 & 27.9 & 17.3 \\
\hline $3: 51: 45$ & 31.7 & 10.7 & 51.7 & 20.2 & 28.8 & 27.9 & 17.2 \\
\hline $3: 52: 00$ & 31.7 & 10.7 & 51.7 & 20.2 & 28.8 & 27.9 & 17.2 \\
\hline $3: 52: 15$ & 31.7 & 10.7 & 51.7 & 20.2 & 28.8 & 27.9 & 17.1 \\
\hline $3: 52: 30$ & 31.7 & 10.7 & 51.7 & 20.2 & 28.8 & 27.9 & 17.3 \\
\hline $3: 52: 45$ & 31.7 & 10.7 & 51.7 & 20.2 & 28.8 & 27.9 & 17.1 \\
\hline $3: 53: 00$ & 31.7 & 10.7 & 51.7 & 20.2 & 28.8 & 27.9 & 17.2 \\
\hline $3: 53: 15$ & 31.7 & 10.7 & 51.8 & 20.2 & 28.8 & 27.9 & 17.1 \\
\hline $3: 53: 30$ & 31.7 & 10.7 & 51.8 & 20.2 & 28.8 & 27.9 & 17.3 \\
\hline $3: 53: 45$ & 31.7 & 10.7 & 51.8 & 20.2 & 28.8 & 27.9 & 17 \\
\hline $3: 54: 00$ & 31.7 & 10.7 & 51.8 & 20.2 & 28.8 & 27.9 & 17.2 \\
\hline $3: 54: 15$ & 31.7 & 10.7 & 51.8 & 20.2 & 28.8 & 27.8 & 17.1 \\
\hline $3: 54: 30$ & 31.7 & 10.7 & 51.8 & 20.2 & 28.8 & 27.9 & 17.1 \\
\hline $3: 54: 45$ & 31.7 & 10.7 & 51.8 & 20.2 & 28.8 & 27.9 & 16.8 \\
\hline $3: 55: 00$ & 31.7 & 10.7 & 51.8 & 20.2 & 28.8 & 27.9 & 16.7 \\
\hline $3: 55: 15$ & 31.7 & 10.7 & 51.8 & 20.2 & 28.8 & 27.9 & 16.8 \\
\hline
\end{tabular}




\begin{tabular}{|c|c|c|c|c|c|c|c|}
\hline $3: 55: 30$ & 31.7 & 10.7 & 51.8 & 20.2 & 28.8 & 27.9 & 16.7 \\
\hline $3: 55: 45$ & 31.7 & 10.7 & 51.8 & 20.2 & 28.8 & 27.9 & 16.7 \\
\hline $3: 56: 00$ & 31.7 & 10.7 & 51.8 & 20.2 & 28.8 & 27.9 & 16.7 \\
\hline $3: 56: 15$ & 31.7 & 10.7 & 51.8 & 20.2 & 28.8 & 27.9 & 16.6 \\
\hline $3: 56: 30$ & 31.8 & 10.7 & 51.8 & 20.2 & 28.8 & 27.9 & 16.5 \\
\hline $3: 56: 45$ & 31.8 & 10.7 & 51.8 & 20.2 & 28.8 & 27.9 & 16.4 \\
\hline $3: 57: 00$ & 31.8 & 10.7 & 51.8 & 20.2 & 28.8 & 27.9 & 16.5 \\
\hline $3: 57: 15$ & 31.7 & 10.7 & 51.8 & 20.1 & 28.8 & 27.8 & 16.5 \\
\hline $3: 57: 30$ & 31.7 & 10.7 & 51.8 & 20.1 & 28.8 & 27.9 & 16.5 \\
\hline $3: 57: 45$ & 31.7 & 10.7 & 51.8 & 20.1 & 28.8 & 27.9 & 16.5 \\
\hline $3: 58: 00$ & 31.7 & 10.7 & 51.8 & 20.1 & 28.8 & 27.9 & 16.2 \\
\hline $3: 58: 15$ & 31.7 & 10.7 & 51.8 & 20.1 & 28.8 & 27.9 & 16.1 \\
\hline $3: 58: 30$ & 31.7 & 10.7 & 51.8 & 20.1 & 28.8 & 27.9 & 16.4 \\
\hline $3: 58: 45$ & 31.7 & 10.7 & 51.8 & 20.1 & 28.8 & 27.9 & 16 \\
\hline $3: 59: 00$ & 31.7 & 10.7 & 51.8 & 20.1 & 28.8 & 27.9 & 16 \\
\hline $3: 59: 15$ & 31.7 & 10.7 & 51.8 & 20.1 & 28.8 & 27.9 & 16.2 \\
\hline $3: 59: 30$ & 31.7 & 10.7 & 51.8 & 20.2 & 28.8 & 27.9 & 16.1 \\
\hline $3: 59: 45$ & 31.7 & 10.7 & 51.8 & 20.1 & 28.8 & 27.9 & 16.3 \\
\hline $4: 00: 00$ & 31.7 & 10.8 & 51.8 & 20.1 & 28.8 & 27.9 & 16.1 \\
\hline $4: 00: 15$ & 31.7 & 10.8 & 51.8 & 20.1 & 28.8 & 27.9 & 16.2 \\
\hline $4: 00: 30$ & 31.7 & 10.8 & 51.8 & 20.1 & 28.8 & 27.9 & 16.3 \\
\hline $4: 00: 45$ & 31.7 & 10.8 & 51.8 & 20.1 & 28.7 & 27.9 & 16.3 \\
\hline $4: 01: 00$ & 31.7 & 10.8 & 51.8 & 20.2 & 28.8 & 27.8 & 16.1 \\
\hline $4: 01: 15$ & 31.6 & 10.8 & 51.8 & 20.2 & 28.8 & 27.9 & 16.5 \\
\hline $4: 01: 30$ & 31.7 & 10.8 & 51.7 & 20.2 & 28.8 & 27.9 & 16.3 \\
\hline $4: 01: 45$ & 31.7 & 10.8 & 51.8 & 20.2 & 28.8 & 27.9 & 16.3 \\
\hline $4: 02: 00$ & 31.7 & 10.9 & 51.8 & 20.2 & 28.8 & 27.9 & 16.4 \\
\hline $4: 02: 15$ & 31.7 & 10.9 & 51.8 & 20.2 & 28.8 & 27.9 & 16.5 \\
\hline $4: 02: 30$ & 31.7 & 10.9 & 51.7 & 20.2 & 28.8 & 27.9 & 16.5 \\
\hline $4: 02: 45$ & 31.7 & 10.9 & 51.7 & 20.2 & 28.8 & 27.9 & 16.5 \\
\hline $4: 03: 00$ & 31.7 & 10.9 & 51.7 & 20.2 & 28.8 & 27.9 & 16.5 \\
\hline $4: 03: 15$ & 31.7 & 10.9 & 51.7 & 20.2 & 28.8 & 27.9 & 16.7 \\
\hline $4: 03: 30$ & 31.6 & 10.8 & 51.7 & 20.2 & 28.8 & 27.9 & 16.7 \\
\hline $4: 03: 45$ & 31.7 & 10.9 & 51.7 & 20.1 & 28.8 & 27.9 & 16.5 \\
\hline $4: 04: 00$ & 31.7 & 10.9 & 51.7 & 20.1 & 28.8 & 27.9 & 16.6 \\
\hline $4: 04: 15$ & 31.7 & 10.9 & 51.7 & 20.1 & 28.8 & 27.9 & 16.7 \\
\hline $4: 04: 30$ & 31.7 & 10.9 & 51.7 & 20.1 & 28.8 & 27.9 & 16.6 \\
\hline $4: 04: 45$ & 31.7 & 10.9 & 51.7 & 20.1 & 28.8 & 27.9 & 16.7 \\
\hline $4: 05: 00$ & 31.7 & 10.8 & 51.8 & 20.1 & 28.8 & 27.9 & 16.9 \\
\hline $4: 05: 15$ & 31.7 & 10.8 & 51.7 & 20.1 & 28.8 & 27.9 & 16.7 \\
\hline $4: 05: 30$ & 31.7 & 10.9 & 51.7 & 20.1 & 28.8 & 27.9 & 16.8 \\
\hline $4: 05: 45$ & 31.7 & 10.9 & 51.7 & 20.1 & 28.8 & 27.9 & 16.7 \\
\hline $4: 06: 00$ & 31.7 & 10.9 & 51.7 & 20.1 & 28.8 & 27.9 & 16.7 \\
\hline
\end{tabular}




\begin{tabular}{|c|c|c|c|c|c|c|c|}
\hline $4: 06: 15$ & 31.7 & 10.9 & 51.7 & 20.1 & 28.8 & 27.9 & 16.8 \\
\hline $4: 06: 30$ & 31.7 & 10.9 & 51.7 & 20.1 & 28.8 & 27.9 & 16.8 \\
\hline $4: 06: 45$ & 31.7 & 10.9 & 51.7 & 20.1 & 28.8 & 27.9 & 16.8 \\
\hline $4: 07: 00$ & 31.7 & 10.9 & 51.7 & 20.1 & 28.8 & 27.9 & 16.9 \\
\hline $4: 07: 15$ & 31.7 & 10.9 & 51.7 & 20.1 & 28.8 & 27.9 & 16.8 \\
\hline $4: 07: 30$ & 31.7 & 10.8 & 51.7 & 20.1 & 28.8 & 27.9 & 16.9 \\
\hline $4: 07: 45$ & 31.6 & 10.8 & 51.7 & 20.1 & 28.8 & 27.9 & 16.9 \\
\hline 4:08:00 & 31.7 & 10.8 & 51.7 & 20.1 & 28.8 & 27.9 & 16.9 \\
\hline $4: 08: 15$ & 31.7 & 10.8 & 51.7 & 20.1 & 28.8 & 27.9 & 17 \\
\hline $4: 08: 30$ & 31.7 & 10.7 & 51.7 & 20.1 & 28.8 & 27.9 & 16.9 \\
\hline $4: 08: 45$ & 31.7 & 10.7 & 51.7 & 20.1 & 28.8 & 27.9 & 16.9 \\
\hline $4: 09: 00$ & 31.7 & 10.7 & 51.7 & 20.1 & 28.8 & 27.9 & 17 \\
\hline $4: 09: 15$ & 31.7 & 10.7 & 51.7 & 20.1 & 28.8 & 27.9 & 17 \\
\hline $4: 09: 30$ & 31.7 & 10.7 & 51.7 & 20.1 & 28.8 & 27.9 & 17 \\
\hline $4: 09: 45$ & 31.7 & 10.7 & 51.7 & 20.1 & 28.8 & 27.9 & 17.1 \\
\hline $4: 10: 00$ & 31.7 & 10.6 & 51.7 & 20.1 & 28.9 & 28 & 17.1 \\
\hline $4: 10: 15$ & 31.7 & 10.6 & 51.7 & 20.1 & 28.8 & 27.9 & 17.1 \\
\hline $4: 10: 30$ & 31.7 & 10.6 & 51.7 & 20 & 28.8 & 27.9 & 17.1 \\
\hline $4: 10: 45$ & 31.7 & 10.6 & 51.7 & 20 & 28.8 & 27.9 & 17.2 \\
\hline $4: 11: 00$ & 31.7 & 10.6 & 51.7 & 20 & 28.8 & 27.9 & 17.2 \\
\hline $4: 11: 15$ & 31.7 & 10.6 & 51.7 & 20 & 28.8 & 27.9 & 17.2 \\
\hline $4: 11: 30$ & 31.7 & 10.6 & 51.7 & 20 & 28.8 & 27.9 & 17 \\
\hline $4: 11: 45$ & 31.7 & 10.6 & 51.7 & 20 & 28.8 & 27.9 & 17.3 \\
\hline $4: 12: 00$ & 31.7 & 10.6 & 51.7 & 20 & 28.8 & 27.9 & 17.3 \\
\hline $4: 12: 15$ & 31.7 & 10.6 & 51.7 & 20.1 & 28.8 & 27.9 & 17.1 \\
\hline $4: 12: 30$ & 31.7 & 10.6 & 51.7 & 20 & 28.8 & 27.9 & 17.1 \\
\hline $4: 12: 45$ & 31.7 & 10.6 & 51.7 & 20 & 28.8 & 27.9 & 17.1 \\
\hline $4: 13: 00$ & 31.7 & 10.5 & 51.7 & 20 & 28.8 & 27.9 & 17.2 \\
\hline $4: 13: 15$ & 31.7 & 10.5 & 51.7 & 20 & 28.8 & 27.9 & 17.3 \\
\hline $4: 13: 30$ & 31.7 & 10.6 & 51.7 & 20 & 28.8 & 27.9 & 17.2 \\
\hline $4: 13: 45$ & 31.7 & 10.5 & 51.7 & 20 & 28.9 & 28 & 17.1 \\
\hline $4: 14: 00$ & 31.7 & 10.5 & 51.7 & 20 & 28.8 & 27.9 & 17.2 \\
\hline $4: 14: 15$ & 31.7 & 10.5 & 51.7 & 20 & 28.8 & 27.9 & 17.2 \\
\hline $4: 14: 30$ & 31.7 & 10.5 & 51.7 & 20 & 28.8 & 27.9 & 17.2 \\
\hline $4: 14: 45$ & 31.7 & 10.6 & 51.7 & 20 & 28.8 & 27.8 & 17.2 \\
\hline $4: 15: 00$ & 31.7 & 10.5 & 51.7 & 20 & 28.8 & 27.9 & 17.3 \\
\hline $4: 15: 15$ & 31.7 & 10.5 & 51.8 & 20.1 & 28.8 & 27.9 & 17.2 \\
\hline $4: 15: 30$ & 31.7 & 10.5 & 51.8 & 20 & 28.8 & 27.9 & 17.2 \\
\hline $4: 15: 45$ & 31.7 & 10.5 & 51.8 & 20 & 28.8 & 27.9 & 17.3 \\
\hline $4: 16: 00$ & 31.7 & 10.5 & 51.8 & 20.1 & 28.8 & 27.9 & 17.2 \\
\hline $4: 16: 15$ & 31.7 & 10.5 & 51.8 & 20 & 28.8 & 27.9 & 17.2 \\
\hline $4: 16: 30$ & 31.7 & 10.5 & 51.8 & 20 & 28.8 & 27.9 & 17.1 \\
\hline $4: 16: 45$ & 31.7 & 10.5 & 51.8 & 20.1 & 28.8 & 27.9 & 17.2 \\
\hline
\end{tabular}




\begin{tabular}{|c|c|c|c|c|c|c|c|}
\hline $4: 17: 00$ & 31.7 & 10.5 & 51.8 & 20.1 & 28.8 & 27.9 & 17.2 \\
\hline $4: 17: 15$ & 31.7 & 10.5 & 51.8 & 20 & 28.8 & 27.8 & 17.1 \\
\hline $4: 17: 30$ & 31.7 & 10.5 & 51.8 & 20 & 28.8 & 27.9 & 17.2 \\
\hline $4: 17: 45$ & 31.7 & 10.5 & 51.8 & 20 & 28.8 & 27.9 & 17.1 \\
\hline $4: 18: 00$ & 31.7 & 10.5 & 51.8 & 20.1 & 28.8 & 27.9 & 17.1 \\
\hline $4: 18: 15$ & 31.7 & 10.5 & 51.8 & 20 & 28.8 & 27.9 & 17 \\
\hline $4: 18: 30$ & 31.7 & 10.5 & 51.8 & 20 & 28.8 & 27.9 & 17.1 \\
\hline $4: 18: 45$ & 31.7 & 10.5 & 51.8 & 20 & 28.8 & 27.9 & 17.1 \\
\hline $4: 19: 00$ & 31.7 & 10.5 & 51.8 & 20.1 & 28.8 & 27.9 & 17.1 \\
\hline $4: 19: 15$ & 31.7 & 10.5 & 51.8 & 20.1 & 28.8 & 27.9 & 17.1 \\
\hline $4: 19: 30$ & 31.7 & 10.5 & 51.8 & 20.1 & 28.8 & 27.9 & 17 \\
\hline $4: 19: 45$ & 31.7 & 10.5 & 51.8 & 20.1 & 28.8 & 27.9 & 16.9 \\
\hline $4: 20: 00$ & 31.8 & 10.5 & 51.8 & 20.1 & 28.9 & 27.9 & 17 \\
\hline $4: 20: 15$ & 31.7 & 10.5 & 51.8 & 20.1 & 28.8 & 27.9 & 17.1 \\
\hline $4: 20: 30$ & 31.7 & 10.4 & 51.8 & 20 & 28.8 & 27.9 & 17.1 \\
\hline $4: 20: 45$ & 31.8 & 10.4 & 51.8 & 20.1 & 28.8 & 27.9 & 16.9 \\
\hline $4: 21: 00$ & 31.7 & 10.5 & 51.8 & 20.1 & 28.8 & 27.9 & 17.1 \\
\hline $4: 21: 15$ & 31.7 & 10.5 & 51.8 & 20.1 & 28.8 & 27.9 & 17.1 \\
\hline $4: 21: 30$ & 31.7 & 10.5 & 51.8 & 20.1 & 28.8 & 27.9 & 17.1 \\
\hline $4: 21: 45$ & 31.6 & 10.5 & 51.8 & 20.1 & 28.8 & 27.9 & 17.3 \\
\hline $4: 22: 00$ & 31.6 & 10.5 & 51.8 & 20.1 & 28.8 & 27.9 & 17.2 \\
\hline $4: 22: 15$ & 31.7 & 10.5 & 51.8 & 20.1 & 28.8 & 27.9 & 17.2 \\
\hline $4: 22: 30$ & 31.7 & 10.5 & 51.8 & 20.1 & 28.8 & 27.9 & 17.3 \\
\hline $4: 22: 45$ & 31.7 & 10.5 & 51.8 & 20.1 & 28.8 & 27.9 & 17.2 \\
\hline $4: 23: 00$ & 31.7 & 10.5 & 51.8 & 20.1 & 28.9 & 28 & 17.2 \\
\hline $4: 23: 15$ & 31.7 & 10.5 & 51.8 & 20.1 & 28.8 & 27.9 & 17.2 \\
\hline $4: 23: 30$ & 31.7 & 10.5 & 51.8 & 20.1 & 28.8 & 27.9 & 17.4 \\
\hline $4: 23: 45$ & 31.7 & 10.5 & 51.9 & 20.1 & 28.9 & 27.9 & 17.2 \\
\hline $4: 24: 00$ & 31.7 & 10.5 & 51.8 & 20.1 & 28.8 & 27.9 & 17.5 \\
\hline $4: 24: 15$ & 31.7 & 10.4 & 51.9 & 20.1 & 28.8 & 27.9 & 17.4 \\
\hline $4: 24: 30$ & 31.7 & 10.4 & 51.8 & 20.1 & 28.8 & 27.9 & 17.3 \\
\hline $4: 24: 45$ & 31.7 & 10.4 & 51.8 & 20.1 & 28.8 & 27.9 & 17.5 \\
\hline $4: 25: 00$ & 31.7 & 10.4 & 51.8 & 20.1 & 28.9 & 28 & 17.6 \\
\hline $4: 25: 15$ & 31.7 & 10.3 & 51.8 & 20.1 & 28.9 & 28 & 17.5 \\
\hline $4: 25: 30$ & 31.8 & 10.4 & 51.8 & 20.1 & 28.8 & 27.9 & 17.5 \\
\hline $4: 25: 45$ & 31.8 & 10.4 & 51.8 & 20.1 & 28.8 & 27.9 & 17.6 \\
\hline $4: 26: 00$ & 31.8 & 10.4 & 51.8 & 20.1 & 28.9 & 28 & 17.4 \\
\hline $4: 26: 15$ & 31.8 & 10.4 & 51.8 & 20.1 & 28.8 & 27.9 & 17.5 \\
\hline $4: 26: 30$ & 31.8 & 10.5 & 51.8 & 20.1 & 28.9 & 28 & 17.4 \\
\hline $4: 26: 45$ & 31.8 & 10.5 & 51.8 & 20.1 & 28.9 & 28 & 17.5 \\
\hline $4: 27: 00$ & 31.8 & 10.5 & 51.8 & 20.2 & 28.8 & 27.9 & 17.6 \\
\hline $4: 27: 15$ & 31.8 & 10.6 & 51.8 & 20.2 & 28.8 & 27.9 & 17.6 \\
\hline $4: 27: 30$ & 31.8 & 10.6 & 51.8 & 20.2 & 28.9 & 27.9 & 17.4 \\
\hline
\end{tabular}




\begin{tabular}{|c|c|c|c|c|c|c|c|}
\hline $4: 27: 45$ & 31.7 & 10.6 & 51.8 & 20.2 & 28.9 & 27.9 & 17.2 \\
\hline $4: 28: 00$ & 31.7 & 10.6 & 51.8 & 20.2 & 28.9 & 28 & 17.3 \\
\hline $4: 28: 15$ & 31.7 & 10.6 & 51.8 & 20.2 & 28.9 & 28 & 17.3 \\
\hline $4: 28: 30$ & 31.7 & 10.5 & 51.9 & 20.2 & 28.9 & 27.9 & 17.3 \\
\hline $4: 28: 45$ & 31.8 & 10.5 & 51.9 & 20.1 & 28.9 & 28 & 17.1 \\
\hline $4: 29: 00$ & 31.8 & 10.5 & 51.9 & 20.1 & 28.9 & 27.9 & 17.2 \\
\hline $4: 29: 15$ & 31.8 & 10.5 & 51.9 & 20.1 & 28.9 & 27.9 & 17 \\
\hline $4: 29: 30$ & 31.7 & 10.5 & 51.9 & 20.1 & 28.8 & 27.9 & 16.8 \\
\hline $4: 29: 45$ & 31.8 & 10.5 & 51.9 & 20.1 & 28.9 & 28 & 16.9 \\
\hline $4: 30: 00$ & 31.8 & 10.5 & 51.9 & 20.1 & 28.9 & 27.9 & 16.8 \\
\hline $4: 30: 15$ & 31.8 & 10.5 & 51.9 & 20.1 & 28.9 & 27.9 & 16.6 \\
\hline $4: 30: 30$ & 31.8 & 10.5 & 51.9 & 20.1 & 28.9 & 28 & 16.8 \\
\hline $4: 30: 45$ & 31.8 & 10.5 & 51.9 & 20 & 28.9 & 27.9 & 16.7 \\
\hline $4: 31: 00$ & 31.8 & 10.5 & 51.9 & 20 & 28.9 & 27.9 & 16.6 \\
\hline $4: 31: 15$ & 31.8 & 10.5 & 51.9 & 20 & 28.9 & 28 & 16.8 \\
\hline $4: 31: 30$ & 31.7 & 10.5 & 51.9 & 20 & 28.9 & 27.9 & 16.6 \\
\hline $4: 31: 45$ & 31.8 & 10.5 & 51.9 & 20 & 28.8 & 27.9 & 16.6 \\
\hline $4: 32: 00$ & 31.7 & 10.4 & 51.9 & 20 & 28.9 & 28 & 16.4 \\
\hline $4: 32: 15$ & 31.8 & 10.5 & 51.8 & 20 & 28.9 & 27.9 & 16.4 \\
\hline $4: 32: 30$ & 31.7 & 10.5 & 51.8 & 20 & 28.8 & 27.9 & 16.5 \\
\hline $4: 32: 45$ & 31.7 & 10.5 & 51.9 & 20 & 28.9 & 28 & 16.5 \\
\hline $4: 33: 00$ & 31.7 & 10.6 & 51.9 & 20.1 & 28.8 & 27.9 & 16.5 \\
\hline $4: 33: 15$ & 31.7 & 10.6 & 51.9 & 20.1 & 28.8 & 27.9 & 16.4 \\
\hline $4: 33: 30$ & 31.7 & 10.6 & 51.8 & 20.1 & 28.8 & 27.9 & 16.5 \\
\hline $4: 33: 45$ & 31.7 & 10.6 & 51.8 & 20.1 & 28.9 & 28 & 16.5 \\
\hline $4: 34: 00$ & 31.8 & 10.7 & 51.8 & 20.1 & 28.8 & 27.9 & 16.5 \\
\hline $4: 34: 15$ & 31.8 & 10.7 & 51.8 & 20.1 & 28.8 & 27.9 & 16.5 \\
\hline $4: 34: 30$ & 31.7 & 10.7 & 51.6 & 20.1 & 28.8 & 27.9 & 16.6 \\
\hline $4: 34: 45$ & 31.7 & 10.7 & 48.2 & 20 & 28.9 & 27.9 & 16.7 \\
\hline $4: 35: 00$ & 31.7 & 10.7 & 43.5 & 20 & 28.8 & 27.9 & 16.5 \\
\hline $4: 35: 15$ & 31.7 & 10.7 & 44.4 & 20.1 & 28.8 & 27.9 & 16.6 \\
\hline $4: 35: 30$ & 31.7 & 10.8 & 44.5 & 20.1 & 28.8 & 27.9 & 16.4 \\
\hline $4: 35: 45$ & 31.7 & 10.7 & 43.6 & 20.1 & 28.8 & 27.9 & 16.1 \\
\hline $4: 36: 00$ & 31.7 & 10.7 & 43.3 & 20.1 & 28.8 & 27.9 & 16.3 \\
\hline $4: 36: 15$ & 31.7 & 10.7 & 43.2 & 20.1 & 28.8 & 27.9 & 16.4 \\
\hline $4: 36: 30$ & 31.7 & 10.7 & 43.1 & 20.1 & 28.8 & 27.9 & 16.3 \\
\hline $4: 36: 45$ & 31.7 & 10.7 & 43.1 & 20.1 & 28.8 & 27.9 & 16.6 \\
\hline $4: 37: 00$ & 31.7 & 10.7 & 43.1 & 20.1 & 28.8 & 27.9 & 16.7 \\
\hline $4: 37: 15$ & 31.7 & 10.7 & 43 & 20.1 & 28.8 & 27.9 & 17 \\
\hline $4: 37: 30$ & 31.7 & 10.8 & 43.1 & 20.1 & 28.8 & 27.9 & 17.1 \\
\hline $4: 37: 45$ & 31.7 & 10.8 & 43.1 & 20.1 & 28.8 & 27.9 & 17.3 \\
\hline $4: 38: 00$ & 31.7 & 10.7 & 43.1 & 20.1 & 28.9 & 27.9 & 17.4 \\
\hline $4: 38: 15$ & 31.7 & 10.8 & 43.2 & 20.1 & 28.8 & 27.9 & 17.5 \\
\hline
\end{tabular}




\begin{tabular}{|c|c|c|c|c|c|c|c|}
\hline $4: 38: 30$ & 31.7 & 10.7 & 43.2 & 20.1 & 28.8 & 27.9 & 17.7 \\
\hline $4: 38: 45$ & 31.7 & 10.7 & 43.2 & 20.1 & 28.8 & 27.9 & 17.8 \\
\hline $4: 39: 00$ & 31.7 & 10.8 & 43.2 & 20.1 & 28.9 & 27.9 & 18 \\
\hline $4: 39: 15$ & 31.7 & 10.8 & 43.3 & 20.1 & 28.8 & 27.9 & 18.1 \\
\hline $4: 39: 30$ & 31.7 & 10.8 & 43.3 & 20.1 & 28.8 & 27.9 & 18.2 \\
\hline $4: 39: 45$ & 31.7 & 10.8 & 43.3 & 20.1 & 28.8 & 27.9 & 18.4 \\
\hline $4: 40: 00$ & 31.8 & 10.8 & 43.4 & 20.1 & 28.8 & 27.9 & 18.5 \\
\hline $4: 40: 15$ & 31.8 & 10.8 & 43.4 & 20.1 & 28.8 & 27.9 & 18.5 \\
\hline $4: 40: 30$ & 31.8 & 10.8 & 43.4 & 20.1 & 28.8 & 27.9 & 18.6 \\
\hline $4: 40: 45$ & 31.7 & 10.8 & 43.5 & 20.1 & 28.8 & 27.9 & 18.7 \\
\hline $4: 41: 00$ & 31.8 & 10.8 & 43.5 & 20.1 & 28.8 & 27.9 & 18.9 \\
\hline $4: 41: 15$ & 31.7 & 10.8 & 43.6 & 20.1 & 28.8 & 27.9 & 18.9 \\
\hline $4: 41: 30$ & 31.7 & 10.8 & 43.6 & 20.1 & 28.8 & 27.9 & 19 \\
\hline $4: 41: 45$ & 31.7 & 10.8 & 43.6 & 20.1 & 28.8 & 27.9 & 19.1 \\
\hline $4: 42: 00$ & 31.7 & 10.8 & 43.6 & 20.1 & 28.8 & 27.9 & 19.2 \\
\hline $4: 42: 15$ & 31.8 & 10.8 & 43.7 & 20.1 & 28.8 & 27.9 & 19.3 \\
\hline $4: 42: 30$ & 31.7 & 10.8 & 43.7 & 20.1 & 28.8 & 27.9 & 19.4 \\
\hline $4: 42: 45$ & 31.8 & 10.8 & 43.7 & 20.1 & 28.8 & 27.9 & 19.5 \\
\hline $4: 43: 00$ & 31.7 & 10.8 & 43.8 & 20.1 & 28.8 & 27.9 & 19.6 \\
\hline $4: 43: 15$ & 31.7 & 10.8 & 43.8 & 20.1 & 28.8 & 27.9 & 19.7 \\
\hline $4: 43: 30$ & 31.7 & 10.8 & 43.8 & 20.1 & 28.8 & 27.9 & 19.7 \\
\hline $4: 43: 45$ & 31.7 & 10.8 & 43.8 & 20.1 & 28.8 & 27.9 & 19.8 \\
\hline $4: 44: 00$ & 31.7 & 10.9 & 43.9 & 20.1 & 28.8 & 27.9 & 19.9 \\
\hline $4: 44: 15$ & 31.8 & 10.9 & 43.9 & 20.1 & 28.8 & 27.9 & 19.9 \\
\hline $4: 44: 30$ & 31.7 & 10.9 & 43.9 & 20.1 & 28.8 & 27.9 & 20 \\
\hline $4: 44: 45$ & 31.7 & 10.8 & 43.9 & 20.1 & 28.9 & 27.9 & 20.1 \\
\hline $4: 45: 00$ & 31.7 & 10.9 & 43.9 & 20.1 & 28.8 & 27.9 & 20.1 \\
\hline $4: 45: 15$ & 31.7 & 10.9 & 43.9 & 20.1 & 28.8 & 27.9 & 20.2 \\
\hline $4: 45: 30$ & 31.7 & 10.9 & 43.9 & 20.1 & 28.8 & 27.9 & 20.3 \\
\hline $4: 45: 45$ & 31.8 & 10.9 & 43.9 & 20.1 & 28.8 & 27.9 & 20.3 \\
\hline $4: 46: 00$ & 31.7 & 10.9 & 43.9 & 20.1 & 28.8 & 27.9 & 20.4 \\
\hline $4: 46: 15$ & 31.8 & 10.9 & 44 & 20.1 & 28.9 & 27.9 & 20.5 \\
\hline $4: 46: 30$ & 31.8 & 10.9 & 44 & 20.1 & 28.8 & 27.9 & 20.5 \\
\hline $4: 46: 45$ & 31.7 & 10.9 & 44 & 20.1 & 28.8 & 27.9 & 20.5 \\
\hline $4: 47: 00$ & 31.8 & 10.9 & 44 & 20.1 & 28.9 & 27.9 & 20.6 \\
\hline $4: 47: 15$ & 31.7 & 10.9 & 44 & 20.1 & 28.8 & 27.8 & 20.7 \\
\hline $4: 47: 30$ & 31.7 & 10.9 & 44 & 20.1 & 28.8 & 27.9 & 20.7 \\
\hline $4: 47: 45$ & 31.7 & 10.9 & 44 & 20.1 & 28.8 & 27.9 & 20.7 \\
\hline $4: 48: 00$ & 31.7 & 10.9 & 44 & 20.1 & 28.8 & 27.9 & 20.8 \\
\hline $4: 48: 15$ & 31.7 & 10.9 & 44.1 & 20.1 & 28.8 & 27.9 & 20.8 \\
\hline $4: 48: 30$ & 31.7 & 10.9 & 44.1 & 20.1 & 28.8 & 27.9 & 20.9 \\
\hline $4: 48: 45$ & 31.7 & 10.9 & 44.1 & 20.1 & 28.8 & 27.9 & 20.9 \\
\hline $4: 49: 00$ & 31.7 & 10.9 & 44.1 & 20.1 & 28.8 & 27.9 & 21 \\
\hline
\end{tabular}




\begin{tabular}{|c|c|c|c|c|c|c|c|}
\hline $4: 49: 15$ & 31.7 & 10.9 & 44.1 & 20.1 & 28.8 & 27.8 & 21 \\
\hline $4: 49: 30$ & 31.7 & 10.9 & 44.1 & 20.1 & 28.8 & 27.9 & 21 \\
\hline $4: 49: 45$ & 31.7 & 10.9 & 44.1 & 20.1 & 28.8 & 27.8 & 21.1 \\
\hline $4: 50: 00$ & 31.7 & 10.9 & 44.2 & 20.1 & 28.8 & 27.8 & 21.1 \\
\hline $4: 50: 15$ & 31.7 & 10.9 & 44.2 & 20.1 & 28.8 & 27.9 & 21.2 \\
\hline $4: 50: 30$ & 31.7 & 10.9 & 44.2 & 20.1 & 28.8 & 27.9 & 21.2 \\
\hline $4: 50: 45$ & 31.7 & 10.9 & 44.2 & 20.1 & 28.8 & 27.9 & 21.2 \\
\hline $4: 51: 00$ & 31.7 & 10.9 & 44.3 & 20.1 & 28.8 & 27.9 & 21.3 \\
\hline $4: 51: 15$ & 31.7 & 10.9 & 44.3 & 20.1 & 28.8 & 27.9 & 21.3 \\
\hline $4: 51: 30$ & 31.7 & 10.9 & 44.3 & 20.1 & 28.8 & 27.9 & 21.3 \\
\hline $4: 51: 45$ & 31.7 & 10.9 & 44.3 & 20.1 & 28.8 & 27.8 & 21.3 \\
\hline $4: 52: 00$ & 31.7 & 10.8 & 44.3 & 20.1 & 28.9 & 27.9 & 21.4 \\
\hline $4: 52: 15$ & 31.8 & 10.9 & 44.3 & 20.1 & 28.8 & 27.8 & 21.4 \\
\hline $4: 52: 30$ & 31.8 & 10.9 & 44.3 & 20.1 & 28.8 & 27.9 & 21.4 \\
\hline $4: 52: 45$ & 31.7 & 10.8 & 44.3 & 20.1 & 28.8 & 27.9 & 21.5 \\
\hline $4: 53: 00$ & 31.7 & 10.9 & 44.3 & 20.1 & 28.7 & 27.8 & 21.5 \\
\hline $4: 53: 15$ & 31.7 & 10.9 & 44.3 & 20.1 & 28.8 & 27.9 & 21.5 \\
\hline $4: 53: 30$ & 31.7 & 10.9 & 44.3 & 20.1 & 28.8 & 27.9 & 21.5 \\
\hline $4: 53: 45$ & 31.7 & 10.9 & 44.4 & 20.1 & 28.8 & 27.8 & 21.5 \\
\hline $4: 54: 00$ & 31.7 & 10.9 & 44.4 & 20.1 & 28.8 & 27.8 & 21.6 \\
\hline $4: 54: 15$ & 31.7 & 10.9 & 44.4 & 20.1 & 28.8 & 27.8 & 21.6 \\
\hline $4: 54: 30$ & 31.7 & 10.8 & 44.4 & 20.1 & 28.8 & 27.9 & 21.6 \\
\hline $4: 54: 45$ & 31.7 & 10.9 & 45.5 & 20.1 & 28.8 & 27.8 & 21.6 \\
\hline $4: 55: 00$ & 31.7 & 10.9 & 45.9 & 20.1 & 28.8 & 27.9 & 21.7 \\
\hline $4: 55: 15$ & 31.7 & 10.9 & 45.9 & 20.1 & 28.8 & 27.9 & 21.7 \\
\hline $4: 55: 30$ & 31.7 & 10.9 & 45.9 & 20.1 & 28.8 & 27.9 & 21.8 \\
\hline $4: 55: 45$ & 31.7 & 10.9 & 45.8 & 20.1 & 28.8 & 27.9 & 21.8 \\
\hline $4: 56: 00$ & 31.7 & 10.9 & 45.8 & 20.1 & 28.8 & 27.9 & 21.8 \\
\hline $4: 56: 15$ & 31.7 & 10.9 & 45.9 & 20.1 & 28.8 & 27.9 & 21.9 \\
\hline $4: 56: 30$ & 31.7 & 10.9 & 45.8 & 20.1 & 28.8 & 27.9 & 21.9 \\
\hline $4: 56: 45$ & 31.8 & 10.8 & 45.8 & 20.1 & 28.9 & 27.9 & 21.9 \\
\hline $4: 57: 00$ & 31.7 & 10.9 & 45.9 & 20.1 & 28.8 & 27.8 & 21.9 \\
\hline $4: 57: 15$ & 31.7 & 10.9 & 45.9 & 20.1 & 28.8 & 27.9 & 22 \\
\hline $4: 57: 30$ & 31.7 & 10.9 & 45.9 & 20.1 & 28.8 & 27.9 & 22 \\
\hline $4: 57: 45$ & 31.7 & 10.8 & 45.9 & 20.1 & 28.8 & 27.9 & 22 \\
\hline $4: 58: 00$ & 31.7 & 10.9 & 45.9 & 20.1 & 28.8 & 27.8 & 22 \\
\hline $4: 58: 15$ & 31.7 & 10.9 & 45.9 & 20.1 & 28.8 & 27.9 & 22 \\
\hline $4: 58: 30$ & 31.7 & 10.8 & 45.9 & 20.1 & 28.8 & 27.9 & 22 \\
\hline $4: 58: 45$ & 31.7 & 10.9 & 46 & 20.1 & 28.8 & 27.8 & 22.1 \\
\hline $4: 59: 00$ & 31.7 & 10.9 & 46 & 20.1 & 28.8 & 27.9 & 22.2 \\
\hline $4: 59: 15$ & 31.8 & 10.8 & 46 & 20.1 & 28.8 & 27.8 & 22.3 \\
\hline $4: 59: 30$ & 31.8 & 10.9 & 46 & 20.1 & 28.7 & 27.8 & 22.3 \\
\hline $4: 59: 45$ & 31.7 & 10.8 & 46.1 & 20.1 & 28.8 & 27.8 & 22.5 \\
\hline
\end{tabular}




\begin{tabular}{|c|c|c|c|c|c|c|c|}
\hline $5: 00: 00$ & 31.8 & 10.9 & 46.1 & 20.1 & 28.8 & 27.8 & 22.5 \\
\hline $5: 00: 15$ & 31.7 & 10.8 & 46 & 20.1 & 28.8 & 27.9 & 22.7 \\
\hline $5: 00: 30$ & 31.6 & 10.9 & 45.4 & 20.2 & 28.8 & 27.2 & 22.5 \\
\hline $5: 00: 45$ & 29.9 & 13.3 & 44.8 & 20.3 & 25.4 & 24.4 & 22.6 \\
\hline $5: 01: 00$ & 27.5 & 15.5 & 44.4 & 20.4 & 23.7 & 23 & 22.6 \\
\hline $5: 01: 15$ & 25.5 & 16.8 & 44 & 20.5 & 22.6 & 22.1 & 22.6 \\
\hline $5: 01: 30$ & 24 & 17.6 & 43.6 & 20.6 & 22 & 21.6 & 22.6 \\
\hline $5: 01: 45$ & 23 & 18.2 & 43.3 & 20.6 & 21.6 & 21.3 & 22.5 \\
\hline $5: 02: 00$ & 22.3 & 18.5 & 42.9 & 20.6 & 21.3 & 21.1 & 22.6 \\
\hline $5: 02: 15$ & 21.8 & 18.8 & 42.6 & 20.7 & 21.1 & 20.9 & 22.5 \\
\hline $5: 02: 30$ & 21.5 & 18.9 & 42.3 & 20.7 & 21 & 20.8 & 22.4 \\
\hline $5: 02: 45$ & 21.3 & 19 & 42 & 20.7 & 20.9 & 20.8 & 22.4 \\
\hline $5: 03: 00$ & 21.1 & 19.1 & 41.6 & 20.7 & 20.9 & 20.7 & 22.4 \\
\hline $5: 03: 15$ & 21 & 19.1 & 41.3 & 20.8 & 20.8 & 20.7 & 22.4 \\
\hline $5: 03: 30$ & 20.9 & 19.1 & 41 & 20.8 & 20.8 & 20.7 & 22.4 \\
\hline $5: 03: 45$ & 20.9 & 19.1 & 40.7 & 20.8 & 20.8 & 20.7 & 22.4 \\
\hline $5: 04: 00$ & 20.8 & 19.1 & 40.4 & 20.8 & 20.8 & 20.6 & 22.4 \\
\hline $5: 04: 15$ & 20.8 & 19.1 & 40.1 & 20.8 & 20.7 & 20.6 & 22.4 \\
\hline $5: 04: 30$ & 20.8 & 19.1 & 39.8 & 20.8 & 20.7 & 20.6 & 22.4 \\
\hline $5: 04: 45$ & 20.7 & 19.1 & 39.5 & 20.8 & 20.7 & 20.6 & 22.4 \\
\hline $5: 05: 00$ & 20.7 & 19.1 & 39.2 & 20.8 & 20.7 & 20.6 & 22.4 \\
\hline $5: 05: 15$ & 20.7 & 19.1 & 38.9 & 20.8 & 20.7 & 20.6 & 22.4 \\
\hline $5: 05: 30$ & 20.7 & 19.1 & 38.6 & 20.8 & 20.7 & 20.6 & 22.4 \\
\hline $5: 05: 45$ & 20.7 & 19.1 & 38.3 & 20.8 & 20.7 & 20.6 & 22.4 \\
\hline 5:06:00 & 20.7 & 19.1 & 38.1 & 20.8 & 20.7 & 20.6 & 22.5 \\
\hline $5: 06: 15$ & 20.7 & 19.1 & 37.8 & 20.8 & 20.7 & 20.6 & 22.4 \\
\hline $5: 06: 30$ & 20.6 & 19 & 37.5 & 20.8 & 20.7 & 20.6 & 22.4 \\
\hline $5: 06: 45$ & 20.6 & 19 & 37.3 & 20.8 & 20.7 & 20.6 & 22.4 \\
\hline $5: 07: 00$ & 20.6 & 19 & 37 & 20.8 & 20.7 & 20.6 & 22.4 \\
\hline $5: 07: 15$ & 20.6 & 19 & 36.7 & 20.8 & 20.7 & 20.6 & 22.5 \\
\hline $5: 07: 30$ & 20.6 & 19 & 36.5 & 20.8 & 20.6 & 20.6 & 22.5 \\
\hline $5: 07: 45$ & 20.6 & 19 & 36.2 & 20.8 & 20.6 & 20.5 & 22.5 \\
\hline 5:08:00 & 20.6 & 19 & 36 & 20.8 & 20.6 & 20.6 & 22.5 \\
\hline $5: 08: 15$ & 20.6 & 19 & 35.7 & 20.8 & 20.6 & 20.6 & 22.5 \\
\hline $5: 08: 30$ & 20.6 & 19 & 35.5 & 20.9 & 20.6 & 20.6 & 22.5 \\
\hline $5: 08: 45$ & 20.6 & 19 & 35.3 & 20.9 & 20.6 & 20.5 & 22.5 \\
\hline 5:09:00 & 20.6 & 19.1 & 35 & 20.9 & 20.6 & 20.5 & 22.5 \\
\hline $5: 09: 15$ & 20.6 & 19.1 & 34.8 & 20.9 & 20.6 & 20.5 & 22.5 \\
\hline $5: 09: 30$ & 20.6 & 19.1 & 34.5 & 20.9 & 20.6 & 20.5 & 22.5 \\
\hline $5: 09: 45$ & 20.7 & 19.1 & 34.3 & 20.9 & 20.6 & 20.5 & 22.5 \\
\hline $5: 10: 00$ & 20.6 & 19.1 & 34.1 & 20.9 & 20.6 & 20.5 & 22.5 \\
\hline $5: 10: 15$ & 20.6 & 19.2 & 33.9 & 20.9 & 20.6 & 20.5 & 22.5 \\
\hline $5: 10: 30$ & 20.6 & 19.2 & 33.7 & 21 & 20.6 & 20.5 & 22.5 \\
\hline
\end{tabular}




\begin{tabular}{|c|c|c|c|c|c|c|c|}
\hline $5: 10: 45$ & 20.6 & 19.2 & 33.4 & 21 & 20.6 & 20.5 & 22.5 \\
\hline $5: 11: 00$ & 20.6 & 19.2 & 33.2 & 21 & 20.6 & 20.5 & 22.5 \\
\hline $5: 11: 15$ & 20.6 & 19.2 & 33 & 21 & 20.6 & 20.5 & 22.5 \\
\hline $5: 11: 30$ & 20.6 & 19.2 & 32.8 & 21 & 20.6 & 20.5 & 22.5 \\
\hline $5: 11: 45$ & 20.6 & 19.2 & 32.6 & 21 & 20.6 & 20.5 & 22.5 \\
\hline $5: 12: 00$ & 20.6 & 19.2 & 32.4 & 21 & 20.6 & 20.5 & 22.5 \\
\hline $5: 12: 15$ & 20.6 & 19.2 & 32.2 & 21.1 & 20.6 & 20.5 & 22.5 \\
\hline $5: 12: 30$ & 20.6 & 19.2 & 32 & 21.1 & 20.6 & 20.5 & 22.5 \\
\hline $5: 12: 45$ & 20.6 & 19.2 & 31.8 & 21.1 & 20.6 & 20.5 & 22.5 \\
\hline $5: 13: 00$ & 20.6 & 19.2 & 31.7 & 21.1 & 20.6 & 20.5 & 22.5 \\
\hline $5: 13: 15$ & 20.6 & 19.2 & 31.5 & 21.1 & 20.6 & 20.5 & 22.5 \\
\hline $5: 13: 30$ & 20.6 & 19.2 & 31.3 & 21.1 & 20.6 & 20.5 & 22.6 \\
\hline $5: 13: 45$ & 20.6 & 19.2 & 31.1 & 21.1 & 20.6 & 20.5 & 22.6 \\
\hline $5: 14: 00$ & 20.6 & 19.2 & 30.9 & 21.1 & 20.6 & 20.5 & 22.6 \\
\hline $5: 14: 15$ & 20.5 & 19.1 & 30.8 & 21.1 & 20.6 & 20.5 & 22.6 \\
\hline $5: 14: 30$ & 20.6 & 19.1 & 30.6 & 21.1 & 20.6 & 20.5 & 22.6 \\
\hline $5: 14: 45$ & 20.6 & 19.1 & 30.4 & 21.1 & 20.6 & 20.5 & 22.6 \\
\hline $5: 15: 00$ & 20.6 & 19.1 & 30.3 & 21.1 & 20.6 & 20.5 & 22.6 \\
\hline $5: 15: 15$ & 20.5 & 19.1 & 30.1 & 21.1 & 20.6 & 20.5 & 22.6 \\
\hline $5: 15: 30$ & 20.5 & 19.1 & 30 & 21.1 & 20.6 & 20.5 & 22.6 \\
\hline $5: 15: 45$ & 20.5 & 19.1 & 29.8 & 21.1 & 20.6 & 20.5 & 22.6 \\
\hline $5: 16: 00$ & 20.5 & 19.1 & 29.7 & 21.1 & 20.6 & 20.5 & 22.6 \\
\hline $5: 16: 15$ & 20.5 & 19.1 & 29.5 & 21.1 & 20.6 & 20.5 & 22.6 \\
\hline $5: 16: 30$ & 20.5 & 19.1 & 29.4 & 21.1 & 20.6 & 20.5 & 22.6 \\
\hline $5: 16: 45$ & 20.5 & 19.1 & 29.2 & 21.1 & 20.6 & 20.5 & 22.6 \\
\hline $5: 17: 00$ & 20.5 & 19.1 & 29.1 & 21.1 & 20.6 & 20.5 & 22.6 \\
\hline $5: 17: 15$ & 20.5 & 19.1 & 29 & 21.1 & 20.6 & 20.5 & 22.6 \\
\hline $5: 17: 30$ & 20.5 & 19.1 & 28.8 & 21.1 & 20.6 & 20.5 & 22.6 \\
\hline $5: 17: 45$ & 20.5 & 19.1 & 28.7 & 21.1 & 20.6 & 20.5 & 22.6 \\
\hline $5: 18: 00$ & 20.5 & 19.1 & 28.6 & 21.1 & 20.6 & 20.5 & 22.6 \\
\hline $5: 18: 15$ & 20.5 & 19.1 & 28.4 & 21.2 & 20.6 & 20.5 & 22.6 \\
\hline $5: 18: 30$ & 20.5 & 19.1 & 28.3 & 21.1 & 20.6 & 20.5 & 22.6 \\
\hline $5: 18: 45$ & 20.5 & 19.1 & 28.2 & 21.1 & 20.6 & 20.5 & 22.6 \\
\hline $5: 19: 00$ & 20.5 & 19.1 & 28.1 & 21.1 & 20.6 & 20.5 & 22.7 \\
\hline $5: 19: 15$ & 20.5 & 19 & 28 & 21.1 & 20.6 & 20.5 & 22.7 \\
\hline $5: 19: 30$ & 20.5 & 19 & 27.8 & 21.1 & 20.6 & 20.5 & 22.7 \\
\hline $5: 19: 45$ & 20.5 & 19 & 27.7 & 21.1 & 20.6 & 20.5 & 22.7 \\
\hline $5: 20: 00$ & 20.5 & 19 & 27.6 & 21.1 & 20.6 & 20.5 & 22.7 \\
\hline $5: 20: 15$ & 20.5 & 19 & 27.5 & 21.1 & 20.6 & 20.5 & 22.7 \\
\hline $5: 20: 30$ & 20.5 & 19 & 27.4 & 21.1 & 20.6 & 20.5 & 22.7 \\
\hline $5: 20: 45$ & 20.5 & 19 & 27.3 & 21.1 & 20.6 & 20.5 & 22.7 \\
\hline $5: 21: 00$ & 20.5 & 19 & 27.2 & 21.1 & 20.5 & 20.5 & 22.7 \\
\hline $5: 21: 15$ & 20.5 & 19 & 27 & 21.1 & 20.7 & 20.8 & 23 \\
\hline
\end{tabular}




\begin{tabular}{|c|c|c|c|c|c|c|c|}
\hline $5: 21: 30$ & 22.2 & 16.4 & 26.8 & 21.1 & 24 & 23.8 & 23.1 \\
\hline $5: 21: 45$ & 24.8 & 14.2 & 26.8 & 21.1 & 25.8 & 25.3 & 23.3 \\
\hline $5: 22: 00$ & 26.8 & 13 & 26.7 & 21.1 & 26.8 & 26.2 & 23.6 \\
\hline $5: 22: 15$ & 28.3 & 12.1 & 26.6 & 21.1 & 27.4 & 26.7 & 23.9 \\
\hline $5: 22: 30$ & 29.3 & 11.6 & 26.6 & 21.1 & 27.8 & 27.1 & 24.3 \\
\hline $5: 22: 45$ & 30 & 11.3 & 26.5 & 21.1 & 28 & 27.2 & 24.5 \\
\hline $5: 23: 00$ & 30.4 & 11.1 & 26.4 & 21.1 & 28.2 & 27.4 & 25.2 \\
\hline $5: 23: 15$ & 30.7 & 11 & 26.4 & 21.1 & 28.3 & 27.5 & 25.4 \\
\hline $5: 23: 30$ & 30.9 & 10.9 & 26.3 & 21.2 & 28.4 & 27.5 & 25.7 \\
\hline $5: 23: 45$ & 31.1 & 10.9 & 26.2 & 21.2 & 28.4 & 27.6 & 26 \\
\hline $5: 24: 00$ & 31.2 & 10.9 & 26.2 & 21.2 & 28.5 & 27.6 & 26.4 \\
\hline $5: 24: 15$ & 31.3 & 10.9 & 26.1 & 21.2 & 28.5 & 27.6 & 26.7 \\
\hline $5: 24: 30$ & 31.3 & 11 & 26.1 & 21.2 & 28.5 & 27.7 & 27 \\
\hline $5: 24: 45$ & 31.4 & 10.9 & 26 & 21.2 & 28.6 & 27.7 & 27.2 \\
\hline $5: 25: 00$ & 31.4 & 11 & 26 & 21.2 & 28.5 & 27.6 & 27.5 \\
\hline $5: 25: 15$ & 31.4 & 11 & 25.9 & 21.3 & 28.6 & 27.7 & 27.9 \\
\hline $5: 25: 30$ & 31.5 & 11 & 25.9 & 21.3 & 28.7 & 27.8 & 28.1 \\
\hline $5: 25: 45$ & 31.5 & 11.1 & 25.8 & 21.3 & 28.6 & 27.7 & 28.4 \\
\hline $5: 26: 00$ & 31.5 & 11.1 & 25.8 & 21.3 & 28.6 & 27.7 & 28.7 \\
\hline $5: 26: 15$ & 31.5 & 11.1 & 25.7 & 21.3 & 28.6 & 27.7 & 29 \\
\hline $5: 26: 30$ & 31.5 & 11.2 & 25.7 & 21.3 & 28.6 & 27.7 & 29.2 \\
\hline $5: 26: 45$ & 31.5 & 11.2 & 25.7 & 21.4 & 28.6 & 27.7 & 29.5 \\
\hline $5: 27: 00$ & 31.5 & 11.2 & 25.7 & 21.4 & 28.6 & 27.7 & 29.8 \\
\hline $5: 27: 15$ & 31.5 & 11.3 & 25.6 & 21.4 & 28.6 & 27.7 & 30.1 \\
\hline $5: 27: 30$ & 31.6 & 11.3 & 25.6 & 21.4 & 28.6 & 27.7 & 30.3 \\
\hline $5: 27: 45$ & 31.6 & 11.3 & 25.6 & 21.4 & 28.6 & 27.7 & 30.6 \\
\hline $5: 28: 00$ & 31.6 & 11.3 & 25.6 & 21.5 & 28.6 & 27.7 & 30.8 \\
\hline $5: 28: 15$ & 31.6 & 11.3 & 25.5 & 21.5 & 28.6 & 27.7 & 31.1 \\
\hline $5: 28: 30$ & 31.6 & 11.3 & 25.5 & 21.5 & 28.6 & 27.7 & 31.3 \\
\hline $5: 28: 45$ & 31.6 & 11.3 & 25.5 & 21.5 & 28.6 & 27.7 & 31.6 \\
\hline $5: 29: 00$ & 31.6 & 11.3 & 25.4 & 21.5 & 28.6 & 27.7 & 31.8 \\
\hline $5: 29: 15$ & 31.6 & 11.4 & 25.4 & 21.6 & 28.6 & 27.8 & 32.1 \\
\hline $5: 29: 30$ & 31.6 & 11.4 & 25.4 & 21.6 & 28.7 & 27.7 & 32.3 \\
\hline $5: 29: 45$ & 31.6 & 11.4 & 25.4 & 21.6 & 28.6 & 27.7 & 32.6 \\
\hline $5: 30: 00$ & 31.6 & 11.4 & 25.4 & 21.7 & 28.7 & 27.8 & 32.8 \\
\hline $5: 30: 15$ & 31.6 & 11.4 & 25.3 & 21.7 & 28.6 & 27.8 & 33.1 \\
\hline $5: 30: 30$ & 31.6 & 11.5 & 25.3 & 21.7 & 28.7 & 27.8 & 33.3 \\
\hline $5: 30: 45$ & 31.6 & 11.5 & 25.3 & 21.7 & 28.6 & 27.7 & 33.6 \\
\hline $5: 31: 00$ & 31.7 & 11.5 & 25.3 & 21.8 & 28.6 & 27.8 & 33.8 \\
\hline $5: 31: 15$ & 31.6 & 11.5 & 25.3 & 21.8 & 28.7 & 27.8 & 34 \\
\hline $5: 31: 30$ & 31.7 & 11.6 & 25.3 & 21.9 & 28.7 & 27.8 & 34.3 \\
\hline $5: 31: 45$ & 31.7 & 11.5 & 48.1 & 20.3 & 28.7 & 27.8 & 34.5 \\
\hline $5: 32: 00$ & 31.6 & 11.5 & 52.4 & 20 & 28.7 & 27.8 & 34.7 \\
\hline
\end{tabular}




\begin{tabular}{|c|c|c|c|c|c|c|c|}
\hline $5: 32: 15$ & 31.6 & 11.6 & 49.8 & 20 & 28.7 & 27.8 & 34.8 \\
\hline $5: 32: 30$ & 31.6 & 11.6 & 47.8 & 20 & 28.7 & 27.8 & 34.8 \\
\hline $5: 32: 45$ & 31.6 & 11.6 & 50.2 & 20 & 28.7 & 27.8 & 34.8 \\
\hline $5: 33: 00$ & 31.7 & 11.6 & 51.7 & 20.1 & 28.7 & 27.8 & 34.9 \\
\hline $5: 33: 15$ & 31.7 & 11.6 & 52 & 20.1 & 28.7 & 27.8 & 34.9 \\
\hline $5: 33: 30$ & 31.7 & 11.6 & 52.1 & 20.1 & 28.7 & 27.8 & 35 \\
\hline $5: 33: 45$ & 31.7 & 11.6 & 52.2 & 20.1 & 28.7 & 27.8 & 35 \\
\hline $5: 34: 00$ & 31.7 & 11.6 & 52.2 & 20.1 & 28.7 & 27.8 & 35.1 \\
\hline $5: 34: 15$ & 31.7 & 11.6 & 52.3 & 20.1 & 28.7 & 27.8 & 35.1 \\
\hline $5: 34: 30$ & 31.7 & 11.6 & 52.3 & 20.1 & 28.7 & 27.8 & 35.1 \\
\hline $5: 34: 45$ & 31.7 & 11.7 & 52.4 & 20.1 & 28.7 & 27.8 & 35.2 \\
\hline $5: 35: 00$ & 31.7 & 11.7 & 52.4 & 20.1 & 28.7 & 27.8 & 35.2 \\
\hline $5: 35: 15$ & 31.7 & 11.7 & 52.4 & 20.1 & 28.7 & 27.8 & 35.2 \\
\hline $5: 35: 30$ & 31.7 & 11.7 & 52.5 & 20.1 & 28.7 & 27.8 & 35.3 \\
\hline $5: 35: 45$ & 31.8 & 11.7 & 52.5 & 20.1 & 28.7 & 27.8 & 35.3 \\
\hline $5: 36: 00$ & 31.7 & 11.7 & 52.6 & 20.1 & 28.7 & 27.8 & 35.3 \\
\hline $5: 36: 15$ & 31.7 & 11.7 & 52.7 & 20.1 & 28.7 & 27.8 & 35.4 \\
\hline $5: 36: 30$ & 31.7 & 11.7 & 52.7 & 20.1 & 28.7 & 27.8 & 35.4 \\
\hline $5: 36: 45$ & 31.7 & 11.7 & 52.8 & 20.1 & 28.7 & 27.8 & 35.4 \\
\hline $5: 37: 00$ & 31.7 & 11.7 & 52.8 & 20.1 & 28.7 & 27.8 & 35.5 \\
\hline $5: 37: 15$ & 31.8 & 11.7 & 52.9 & 20.1 & 28.7 & 27.8 & 35.5 \\
\hline $5: 37: 30$ & 31.8 & 11.7 & 52.9 & 20.1 & 28.7 & 27.8 & 35.5 \\
\hline $5: 37: 45$ & 31.8 & 11.8 & 53 & 20.1 & 28.7 & 27.8 & 35.6 \\
\hline $5: 38: 00$ & 31.8 & 11.8 & 53.1 & 20.1 & 28.7 & 27.8 & 35.6 \\
\hline $5: 38: 15$ & 31.8 & 11.8 & 53.1 & 20.1 & 28.7 & 27.8 & 35.6 \\
\hline $5: 38: 30$ & 31.8 & 11.8 & 53.2 & 20.1 & 28.7 & 27.8 & 35.7 \\
\hline $5: 38: 45$ & 31.8 & 11.9 & 53.3 & 20.1 & 28.7 & 27.8 & 35.7 \\
\hline $5: 39: 00$ & 31.8 & 11.9 & 53.3 & 20.1 & 28.7 & 27.8 & 35.7 \\
\hline $5: 39: 15$ & 31.8 & 11.9 & 53.4 & 20.1 & 28.7 & 27.8 & 35.8 \\
\hline $5: 39: 30$ & 31.8 & 11.9 & 53.4 & 20.1 & 28.7 & 27.8 & 35.8 \\
\hline $5: 39: 45$ & 31.8 & 11.9 & 53.4 & 20.1 & 28.7 & 27.8 & 35.8 \\
\hline $5: 40: 00$ & 31.8 & 12 & 53.5 & 20.1 & 28.7 & 27.8 & 35.9 \\
\hline $5: 40: 15$ & 31.8 & 12 & 53.5 & 20.1 & 28.7 & 27.8 & 35.6 \\
\hline $5: 40: 30$ & 31.8 & 12 & 53.6 & 20.1 & 28.7 & 27.8 & 36 \\
\hline $5: 40: 45$ & 31.8 & 12 & 53.6 & 20.1 & 28.7 & 27.8 & 35.9 \\
\hline $5: 41: 00$ & 31.8 & 12 & 53.7 & 20.1 & 28.7 & 27.8 & 35.7 \\
\hline $5: 41: 15$ & 31.9 & 12 & 53.7 & 20.1 & 28.7 & 27.8 & 35.6 \\
\hline $5: 41: 30$ & 31.8 & 12 & 53.7 & 20.1 & 28.7 & 27.8 & 35.7 \\
\hline $5: 41: 45$ & 31.8 & 12 & 53.7 & 20.1 & 28.7 & 27.8 & 35.8 \\
\hline $5: 42: 00$ & 31.8 & 12.1 & 53.7 & 20.1 & 28.7 & 27.8 & 35.7 \\
\hline $5: 42: 15$ & 31.8 & 12.1 & 53.7 & 20.1 & 28.7 & 27.8 & 35.5 \\
\hline $5: 42: 30$ & 31.9 & 12.1 & 53.7 & 20.1 & 28.7 & 27.8 & 35.6 \\
\hline $5: 42: 45$ & 31.8 & 12.1 & 53.7 & 20.1 & 28.7 & 27.8 & 35.6 \\
\hline
\end{tabular}




\begin{tabular}{|c|c|c|c|c|c|c|c|}
\hline $5: 43: 00$ & 31.8 & 12.1 & 53.8 & 20.1 & 28.7 & 27.8 & 35.5 \\
\hline $5: 43: 15$ & 31.8 & 12.1 & 53.8 & 20.1 & 28.7 & 27.8 & 35.4 \\
\hline $5: 43: 30$ & 31.9 & 12.1 & 53.8 & 20.1 & 28.7 & 27.8 & 35.4 \\
\hline $5: 43: 45$ & 31.9 & 12.1 & 53.8 & 20.1 & 28.7 & 27.8 & 35.3 \\
\hline $5: 44: 00$ & 31.9 & 12.1 & 53.8 & 20.1 & 28.7 & 27.8 & 35.2 \\
\hline $5: 44: 15$ & 31.9 & 12.2 & 53.8 & 20.1 & 28.7 & 27.8 & 35.1 \\
\hline $5: 44: 30$ & 31.9 & 12.2 & 53.8 & 20.1 & 28.7 & 27.8 & 35.1 \\
\hline $5: 44: 45$ & 31.9 & 12.2 & 53.8 & 20.1 & 28.8 & 27.8 & 35.1 \\
\hline $5: 45: 00$ & 31.9 & 12.2 & 53.8 & 20.1 & 28.7 & 27.8 & 35 \\
\hline $5: 45: 15$ & 31.9 & 12.3 & 53.8 & 20.1 & 28.7 & 27.8 & 34.9 \\
\hline $5: 45: 30$ & 31.9 & 12.3 & 53.8 & 20.2 & 28.8 & 27.9 & 34.9 \\
\hline $5: 45: 45$ & 31.9 & 12.3 & 53.8 & 20.1 & 28.7 & 27.8 & 34.7 \\
\hline $5: 46: 00$ & 31.9 & 12.3 & 53.8 & 20.1 & 28.7 & 27.8 & 34.7 \\
\hline $5: 46: 15$ & 31.9 & 12.3 & 53.8 & 20.1 & 28.7 & 27.8 & 34.7 \\
\hline $5: 46: 30$ & 31.9 & 12.3 & 53.8 & 20.2 & 28.7 & 27.8 & 34.6 \\
\hline $5: 46: 45$ & 31.9 & 12.3 & 53.8 & 20.1 & 28.8 & 27.8 & 34.5 \\
\hline $5: 47: 00$ & 31.9 & 12.3 & 53.8 & 20.1 & 28.7 & 27.8 & 34.4 \\
\hline $5: 47: 15$ & 31.8 & 12.4 & 53.8 & 20.1 & 28.7 & 27.8 & 34.3 \\
\hline $5: 47: 30$ & 31.9 & 12.4 & 53.8 & 20.1 & 28.8 & 27.8 & 34.2 \\
\hline $5: 47: 45$ & 31.8 & 12.4 & 53.8 & 20.1 & 28.8 & 27.8 & 34.2 \\
\hline $5: 48: 00$ & 31.8 & 12.4 & 53.8 & 20.2 & 28.7 & 27.8 & 34.1 \\
\hline $5: 48: 15$ & 31.8 & 12.4 & 53.8 & 20.1 & 28.7 & 27.8 & 34 \\
\hline $5: 48: 30$ & 31.8 & 12.4 & 53.8 & 20.1 & 28.7 & 27.8 & 34 \\
\hline $5: 48: 45$ & 31.8 & 12.4 & 53.8 & 20.1 & 28.7 & 27.8 & 34 \\
\hline $5: 49: 00$ & 31.8 & 12.4 & 53.8 & 20.1 & 28.7 & 27.8 & 33.8 \\
\hline $5: 49: 15$ & 31.8 & 12.4 & 53.8 & 20.1 & 28.7 & 27.8 & 33.7 \\
\hline $5: 49: 30$ & 31.8 & 12.4 & 53.8 & 20.1 & 28.7 & 27.8 & 33.7 \\
\hline $5: 49: 45$ & 31.8 & 12.3 & 53.8 & 20.1 & 28.8 & 27.9 & 33.6 \\
\hline $5: 50: 00$ & 31.9 & 12.4 & 53.8 & 20.2 & 28.7 & 27.8 & 33.5 \\
\hline $5: 50: 15$ & 31.9 & 12.4 & 53.8 & 20.2 & 28.7 & 27.8 & 33.5 \\
\hline $5: 50: 30$ & 31.8 & 12.4 & 53.8 & 20.2 & 28.8 & 27.9 & 33.4 \\
\hline $5: 50: 45$ & 31.8 & 12.4 & 53.8 & 20.1 & 28.7 & 27.8 & 33.4 \\
\hline $5: 51: 00$ & 31.8 & 12.4 & 53.8 & 20.2 & 28.8 & 27.9 & 33.3 \\
\hline $5: 51: 15$ & 31.8 & 12.4 & 53.8 & 20.1 & 28.8 & 27.8 & 33.1 \\
\hline $5: 51: 30$ & 31.8 & 12.4 & 53.7 & 20.2 & 28.8 & 27.9 & 33.1 \\
\hline $5: 51: 45$ & 31.9 & 12.4 & 53.8 & 20.1 & 28.8 & 27.9 & 33.1 \\
\hline $5: 52: 00$ & 31.9 & 12.4 & 53.8 & 20.1 & 28.8 & 27.9 & 33 \\
\hline $5: 52: 15$ & 31.9 & 12.4 & 53.8 & 20.2 & 28.8 & 27.9 & 33 \\
\hline $5: 52: 30$ & 31.9 & 12.4 & 53.8 & 20.1 & 28.8 & 27.8 & 32.9 \\
\hline $5: 52: 45$ & 31.9 & 12.4 & 53.7 & 20.1 & 28.8 & 27.9 & 32.8 \\
\hline $5: 53: 00$ & 31.9 & 12.4 & 53.6 & 20.1 & 28.8 & 27.9 & 32.7 \\
\hline $5: 53: 15$ & 31.9 & 12.5 & 53.5 & 20.1 & 28.8 & 27.9 & 32.7 \\
\hline $5: 53: 30$ & 31.9 & 12.5 & 53.5 & 20.1 & 28.8 & 27.9 & 32.6 \\
\hline
\end{tabular}




\begin{tabular}{|c|c|c|c|c|c|c|c|}
\hline $5: 53: 45$ & 31.9 & 12.5 & 53.4 & 20.1 & 28.8 & 27.9 & 32.6 \\
\hline $5: 54: 00$ & 31.9 & 12.5 & 53.4 & 20.1 & 28.8 & 27.9 & 32.5 \\
\hline $5: 54: 15$ & 31.9 & 12.5 & 53.4 & 20.1 & 28.8 & 27.9 & 32.4 \\
\hline $5: 54: 30$ & 31.9 & 12.5 & 53.4 & 20.1 & 28.8 & 27.9 & 32.3 \\
\hline $5: 54: 45$ & 31.9 & 12.6 & 53.4 & 20.1 & 28.8 & 27.9 & 32.3 \\
\hline $5: 55: 00$ & 31.9 & 12.6 & 53.4 & 20.1 & 28.8 & 27.9 & 32.3 \\
\hline $5: 55: 15$ & 31.9 & 12.6 & 53.4 & 20.1 & 28.8 & 27.9 & 32.2 \\
\hline $5: 55: 30$ & 31.9 & 12.6 & 53.4 & 20.1 & 28.8 & 27.9 & 32.2 \\
\hline $5: 55: 45$ & 31.9 & 12.6 & 53.4 & 20.1 & 28.8 & 27.9 & 32.1 \\
\hline $5: 56: 00$ & 31.9 & 12.6 & 53.4 & 20.1 & 28.8 & 27.9 & 32 \\
\hline $5: 56: 15$ & 31.9 & 12.7 & 53.4 & 20.1 & 28.8 & 27.9 & 32 \\
\hline $5: 56: 30$ & 31.9 & 12.7 & 53.4 & 20.1 & 28.8 & 27.9 & 31.9 \\
\hline $5: 56: 45$ & 31.9 & 12.7 & 53.4 & 20.1 & 28.8 & 27.9 & 31.9 \\
\hline $5: 57: 00$ & 31.9 & 12.7 & 53.3 & 20.1 & 28.8 & 27.9 & 31.8 \\
\hline $5: 57: 15$ & 31.9 & 12.7 & 53.4 & 20.1 & 28.8 & 27.9 & 31.8 \\
\hline $5: 57: 30$ & 31.9 & 12.7 & 53.4 & 20.1 & 28.8 & 27.9 & 31.8 \\
\hline $5: 57: 45$ & 31.9 & 12.7 & 53.4 & 20.1 & 28.8 & 27.9 & 31.7 \\
\hline $5: 58: 00$ & 31.9 & 12.7 & 53.3 & 20.2 & 28.8 & 27.9 & 31.7 \\
\hline $5: 58: 15$ & 31.9 & 12.7 & 53.4 & 20.1 & 28.8 & 27.9 & 31.6 \\
\hline $5: 58: 30$ & 31.9 & 12.7 & 53.3 & 20.1 & 28.8 & 27.9 & 31.6 \\
\hline $5: 58: 45$ & 31.9 & 12.7 & 53.3 & 20.2 & 28.8 & 27.9 & 31.6 \\
\hline $5: 59: 00$ & 31.9 & 12.7 & 53.3 & 20.1 & 28.8 & 27.9 & 31.6 \\
\hline $5: 59: 15$ & 31.9 & 12.7 & 53.3 & 20.1 & 28.8 & 27.9 & 31.5 \\
\hline $5: 59: 30$ & 31.9 & 12.7 & 53.3 & 20.1 & 28.8 & 27.9 & 31.4 \\
\hline $5: 59: 45$ & 31.9 & 12.7 & 53.3 & 20.1 & 28.8 & 27.9 & 31.4 \\
\hline $6: 00: 00$ & 31.9 & 12.6 & 53.3 & 20.1 & 28.9 & 27.9 & 31.4 \\
\hline $6: 00: 15$ & 31.9 & 12.6 & 53.3 & 20.2 & 28.8 & 27.9 & 31.4 \\
\hline $6: 00: 30$ & 31.9 & 12.6 & 53.3 & 20.1 & 28.8 & 27.9 & 31.3 \\
\hline $6: 00: 45$ & 31.9 & 12.6 & 53.3 & 20.2 & 28.8 & 27.9 & 31.3 \\
\hline $6: 01: 00$ & 31.9 & 12.6 & 53.3 & 20.2 & 28.9 & 28 & 31.3 \\
\hline $6: 01: 15$ & 31.9 & 12.6 & 53.3 & 20.2 & 28.9 & 27.9 & 31.4 \\
\hline $6: 01: 30$ & 31.9 & 12.6 & 53.4 & 20.1 & 28.8 & 27.9 & 31.5 \\
\hline $6: 01: 45$ & 31.9 & 12.6 & 53.4 & 20.2 & 28.9 & 28 & 31.5 \\
\hline $6: 02: 00$ & 31.9 & 12.6 & 53.4 & 20.2 & 28.9 & 28 & 31.6 \\
\hline $6: 02: 15$ & 31.9 & 12.6 & 53.4 & 20.1 & 28.8 & 27.9 & 31.7 \\
\hline $6: 02: 30$ & 31.9 & 12.6 & 53.4 & 20.1 & 28.9 & 27.9 & 31.8 \\
\hline $6: 02: 45$ & 31.9 & 12.6 & 53.5 & 20.1 & 28.8 & 27.9 & 31.9 \\
\hline $6: 03: 00$ & 31.9 & 12.6 & 53.5 & 20.1 & 28.8 & 27.9 & 32 \\
\hline $6: 03: 15$ & 31.9 & 12.6 & 53.5 & 20.2 & 28.9 & 28 & 32.1 \\
\hline $6: 03: 30$ & 31.9 & 12.6 & 53.6 & 20.2 & 28.8 & 27.9 & 32.1 \\
\hline $6: 03: 45$ & 31.9 & 12.6 & 53.7 & 20.1 & 28.8 & 27.9 & 32.2 \\
\hline $6: 04: 00$ & 31.9 & 12.5 & 53.7 & 20.1 & 28.9 & 28 & 32.3 \\
\hline $6: 04: 15$ & 31.9 & 12.5 & 53.7 & 20.2 & 28.9 & 28 & 32.4 \\
\hline
\end{tabular}




\begin{tabular}{|c|c|c|c|c|c|c|c|}
\hline $6: 04: 30$ & 31.9 & 12.5 & 53.8 & 20.2 & 28.9 & 28 & 32.5 \\
\hline $6: 04: 45$ & 31.9 & 12.5 & 53.8 & 20.2 & 28.9 & 27.9 & 32.6 \\
\hline $6: 05: 00$ & 31.9 & 12.5 & 53.9 & 20.1 & 28.8 & 27.9 & 32.6 \\
\hline $6: 05: 15$ & 31.9 & 12.5 & 53.9 & 20.1 & 28.9 & 27.9 & 32.7 \\
\hline $6: 05: 30$ & 31.9 & 12.5 & 53.9 & 20.1 & 28.9 & 27.9 & 32.8 \\
\hline $6: 05: 45$ & 31.9 & 12.5 & 54 & 20.2 & 28.9 & 28 & 32.9 \\
\hline $6: 06: 00$ & 31.9 & 12.4 & 54 & 20.2 & 28.9 & 28 & 33 \\
\hline $6: 06: 15$ & 31.9 & 12.4 & 54.1 & 20.1 & 28.9 & 28 & 33 \\
\hline $6: 06: 30$ & 31.9 & 12.5 & 54.1 & 20.2 & 28.9 & 28 & 33.1 \\
\hline $6: 06: 45$ & 31.9 & 12.5 & 54.2 & 20.1 & 28.9 & 28 & 33.2 \\
\hline $6: 07: 00$ & 31.9 & 12.4 & 54.2 & 20.1 & 28.9 & 28 & 33.3 \\
\hline $6: 07: 15$ & 31.9 & 12.4 & 54.3 & 20.1 & 28.9 & 28 & 33.3 \\
\hline $6: 07: 30$ & 31.9 & 12.4 & 54.3 & 20.2 & 28.9 & 28 & 33.4 \\
\hline $6: 07: 45$ & 31.9 & 12.4 & 54.3 & 20.2 & 28.9 & 28 & 33.5 \\
\hline $6: 08: 00$ & 31.9 & 12.4 & 54.4 & 20.2 & 28.9 & 28 & 33.6 \\
\hline $6: 08: 15$ & 31.9 & 12.4 & 54.4 & 20.2 & 28.9 & 28 & 33.6 \\
\hline $6: 08: 30$ & 31.9 & 12.4 & 54.4 & 20.2 & 28.9 & 28 & 33.7 \\
\hline $6: 08: 45$ & 31.9 & 12.3 & 54.5 & 20.2 & 28.9 & 28 & 33.6 \\
\hline $6: 09: 00$ & 31.9 & 12.3 & 54.5 & 20.2 & 28.9 & 28 & 33.8 \\
\hline $6: 09: 15$ & 31.9 & 12.3 & 54.6 & 20.2 & 28.9 & 28 & 33.6 \\
\hline $6: 09: 30$ & 31.9 & 12.3 & 54.6 & 20.2 & 28.9 & 28 & 33.6 \\
\hline $6: 09: 45$ & 31.9 & 12.4 & 54.6 & 20.2 & 28.9 & 28 & 33.7 \\
\hline $6: 10: 00$ & 31.9 & 12.4 & 54.6 & 20.2 & 28.9 & 28 & 33.7 \\
\hline $6: 10: 15$ & 31.9 & 12.4 & 54.6 & 20.2 & 28.9 & 28 & 33.5 \\
\hline $6: 10: 30$ & 31.9 & 12.4 & 54.6 & 20.2 & 28.9 & 28 & 33.6 \\
\hline $6: 10: 45$ & 31.9 & 12.4 & 54.6 & 20.2 & 28.9 & 28 & 33.5 \\
\hline $6: 11: 00$ & 31.9 & 12.4 & 54.4 & 20.2 & 28.9 & 28 & 33.4 \\
\hline $6: 11: 15$ & 30.7 & 14.7 & 53.6 & 20.3 & 25.8 & 25.1 & 33.1 \\
\hline $6: 11: 30$ & 28.3 & 16.9 & 52.8 & 20.4 & 23.9 & 23.4 & 33 \\
\hline $6: 11: 45$ & 26.2 & 18.3 & 52.1 & 20.5 & 22.8 & 22.5 & 32.9 \\
\hline $6: 12: 00$ & 24.6 & 19.1 & 51.6 & 20.5 & 22.2 & 21.9 & 32.8 \\
\hline $6: 12: 15$ & 23.4 & 19.7 & 51.1 & 20.6 & 21.7 & 21.5 & 32.8 \\
\hline $6: 12: 30$ & 22.7 & 20 & 50.6 & 20.6 & 21.5 & 21.3 & 32.7 \\
\hline $6: 12: 45$ & 22.2 & 20.2 & 50.1 & 20.7 & 21.3 & 21.1 & 32.5 \\
\hline $6: 13: 00$ & 21.8 & 20.3 & 49.6 & 20.7 & 21.2 & 21 & 32.4 \\
\hline $6: 13: 15$ & 21.5 & 20.4 & 49.2 & 20.7 & 21.1 & 21 & 32.4 \\
\hline $6: 13: 30$ & 21.4 & 20.4 & 48.7 & 20.8 & 21 & 20.9 & 32.2 \\
\hline $6: 13: 45$ & 21.3 & 20.4 & 48.3 & 20.8 & 21 & 20.9 & 32.1 \\
\hline $6: 14: 00$ & 21.2 & 20.4 & 47.8 & 20.8 & 21 & 20.9 & 32 \\
\hline $6: 14: 15$ & 21.2 & 20.4 & 47.4 & 20.8 & 20.9 & 20.8 & 31.9 \\
\hline $6: 14: 30$ & 21.2 & 20.4 & 46.9 & 20.9 & 20.9 & 20.8 & 31.8 \\
\hline $6: 14: 45$ & 21.1 & 20.4 & 46.5 & 20.9 & 20.9 & 20.8 & 31.7 \\
\hline $6: 15: 00$ & 21.1 & 20.4 & 46 & 20.9 & 20.9 & 20.8 & 31.5 \\
\hline
\end{tabular}




\begin{tabular}{|c|c|c|c|c|c|c|c|}
\hline $6: 15: 15$ & 21.1 & 20.3 & 45.5 & 21 & 21 & 20.8 & 31.4 \\
\hline $6: 15: 30$ & 21 & 20.2 & 45 & 21 & 21 & 20.8 & 31.3 \\
\hline $6: 15: 45$ & 21 & 20 & 44.6 & 21 & 21 & 20.8 & 31.2 \\
\hline $6: 16: 00$ & 21 & 19.9 & 44.1 & 21 & 20.9 & 20.8 & 31 \\
\hline $6: 16: 15$ & 21 & 19.8 & 43.7 & 21.1 & 20.9 & 20.8 & 30.9 \\
\hline $6: 16: 30$ & 21 & 19.7 & 43.3 & 21.1 & 20.9 & 20.8 & 30.8 \\
\hline $6: 16: 45$ & 21 & 19.6 & 42.8 & 21.1 & 20.8 & 20.8 & 30.7 \\
\hline $6: 17: 00$ & 21 & 19.5 & 42.4 & 21.1 & 20.8 & 20.8 & 30.5 \\
\hline $6: 17: 15$ & 21 & 19.4 & 42 & 21.1 & 20.8 & 20.8 & 30.5 \\
\hline $6: 17: 30$ & 21 & 19.2 & 41.7 & 21.1 & 20.8 & 20.8 & 30.3 \\
\hline $6: 17: 45$ & 21 & 19.1 & 41.3 & 21.2 & 20.7 & 20.8 & 30.2 \\
\hline $6: 18: 00$ & 20.9 & 19 & 40.9 & 21.2 & 20.7 & 20.8 & 30.1 \\
\hline $6: 18: 15$ & 20.9 & 18.9 & 40.6 & 21.2 & 20.7 & 20.8 & 30 \\
\hline $6: 18: 30$ & 20.9 & 18.8 & 40.3 & 21.2 & 20.7 & 20.8 & 29.8 \\
\hline $6: 18: 45$ & 20.9 & 18.7 & 39.9 & 21.2 & 20.6 & 20.8 & 29.7 \\
\hline $6: 19: 00$ & 20.9 & 18.6 & 39.6 & 21.2 & 20.6 & 20.7 & 29.6 \\
\hline $6: 19: 15$ & 20.9 & 18.5 & 39.3 & 21.2 & 20.6 & 20.7 & 29.5 \\
\hline $6: 19: 30$ & 21 & 18.4 & 39 & 21.2 & 20.5 & 20.7 & 29.4 \\
\hline $6: 19: 45$ & 21 & 18.3 & 38.7 & 21.2 & 20.5 & 20.7 & 29.3 \\
\hline $6: 20: 00$ & 21 & 18.3 & 38.4 & 21.2 & 20.5 & 20.6 & 29.2 \\
\hline $6: 20: 15$ & 21 & 18.2 & 38.1 & 21.2 & 20.5 & 20.6 & 29.1 \\
\hline $6: 20: 30$ & 21.1 & 18.1 & 37.9 & 21.2 & 20.4 & 20.6 & 29 \\
\hline $6: 20: 45$ & 21.1 & 18 & 37.6 & 21.2 & 20.4 & 20.5 & 29 \\
\hline $6: 21: 00$ & 21.1 & 18 & 37.3 & 21.3 & 20.4 & 20.5 & 29.1 \\
\hline $6: 21: 15$ & 21.2 & 17.9 & 37.1 & 21.2 & 20.4 & 20.5 & 29 \\
\hline $6: 21: 30$ & 21.2 & 17.8 & 36.8 & 21.2 & 20.3 & 20.4 & 29 \\
\hline $6: 21: 45$ & 21.2 & 17.8 & 36.6 & 21.2 & 20.3 & 20.4 & 29 \\
\hline $6: 22: 00$ & 21.2 & 17.7 & 36.3 & 21.2 & 20.3 & 20.4 & 29 \\
\hline $6: 22: 15$ & 21.3 & 17.7 & 36.1 & 21.2 & 20.3 & 20.3 & 29 \\
\hline $6: 22: 30$ & 21.3 & 17.6 & 35.9 & 21.2 & 20.3 & 20.3 & 29 \\
\hline $6: 22: 45$ & 21.3 & 17.5 & 34.7 & 21.3 & 20.2 & 20.3 & 29 \\
\hline $6: 23: 00$ & 21.3 & 17.5 & 34.8 & 21.3 & 20.2 & 20.2 & 29.1 \\
\hline $6: 23: 15$ & 21.4 & 17.4 & 34.9 & 21.3 & 20.2 & 20.2 & 29 \\
\hline $6: 23: 30$ & 21.4 & 17.4 & 39.9 & 20 & 20.2 & 20.2 & 29.1 \\
\hline $6: 23: 45$ & 21.4 & 17.3 & 41.6 & 20.2 & 20.2 & 20.2 & 29.1 \\
\hline $6: 24: 00$ & 21.5 & 17.3 & 41.7 & 20.9 & 20.1 & 20.1 & 29.1 \\
\hline $6: 24: 15$ & 21.5 & 17.2 & 41.8 & 21 & 20.1 & 20.1 & 29.1 \\
\hline $6: 24: 30$ & 21.5 & 17.2 & 41.8 & 20.9 & 20.1 & 20.1 & 29 \\
\hline $6: 24: 45$ & 21.5 & 17.1 & 41.8 & 20.8 & 20.1 & 20.1 & 29 \\
\hline $6: 25: 00$ & 21.5 & 17.1 & 41.7 & 20.6 & 20 & 20 & 29 \\
\hline $6: 25: 15$ & 21.6 & 17.1 & 41.7 & 20.5 & 20 & 20 & 29 \\
\hline $6: 25: 30$ & 21.6 & 17 & 41.6 & 20.4 & 20 & 20 & 29 \\
\hline $6: 25: 45$ & 21.6 & 17 & 41.6 & 20.3 & 20 & 20 & 29 \\
\hline
\end{tabular}




\begin{tabular}{l|lllllll}
$6: 26: 00$ & 21.6 & 17 & 41.5 & 20.2 & 20 & 19.9 & 28.9 \\
$6: 26: 15$ & 21.7 & 16.9 & 41.1 & 20.2 & 20 & 19.9 & 28.9 \\
$6: 26: 30$ & 21.7 & 16.9 & 39.9 & 20.6 & 20 & 19.9 & 28.9 \\
$6: 26: 45$ & 21.7 & 16.9 & 39.2 & 20.7 & 19.9 & 19.9 & 28.9 \\
$6: 27: 00$ & 21.7 & 16.8 & 38.7 & 20.8 & 19.9 & 19.8 & 28.9 \\
$6: 27: 15$ & 21.7 & 16.8 & 41.2 & 20 & 19.9 & 19.8 & 28.8 \\
$6: 27: 30$ & 21.7 & 16.8 & 37.6 & 20 & 19.9 & 19.8 & 28.8 \\
$6: 27: 45$ & 21.8 & 16.8 & 33.7 & 20 & 19.9 & 19.8 & 28.7 \\
$6: 28: 00$ & 21.8 & 16.7 & 32.4 & 20 & 19.9 & 19.8 & 28.6 \\
$6: 28: 15$ & 21.8 & 16.7 & 31.6 & 20 & 19.8 & 19.7 & 28.6 \\
$6: 28: 30$ & 21.8 & 16.7 & 31.3 & 20.1 & 19.8 & 19.7 & 28.5 \\
$6: 28: 45$ & 21.8 & 16.6 & 31 & 20.1 & 19.8 & 19.7 & 28.4 \\
$6: 29: 00$ & 21.8 & 16.6 & 30.5 & 20.2 & 19.8 & 19.7 & 28.4 \\
$6: 29: 15$ & 21.8 & 16.6 & 30.9 & 20.1 & 19.8 & 19.7 & 28.4 \\
$6: 29: 30$ & 21.8 & 16.6 & 31.1 & 19.8 & 19.8 & 19.6 & 28.4 \\
$6: 29: 45$ & 21.9 & 16.5 & 31.2 & 19.8 & 19.8 & 19.6 & 28.3 \\
$6: 30: 00$ & 21.9 & 16.5 & 36.9 & 19.7 & 19.8 & 19.6 & 28.3 \\
$6: 30: 15$ & 21.9 & 16.5 & 32.1 & 19.7 & 19.7 & 19.6 & 28.2 \\
$6: 30: 30$ & 21.9 & 16.5 & 31.1 & 19.7 & 19.7 & 19.6 & 28.1 \\
$6: 30: 45$ & 21.9 & 16.4 & 30.5 & 19.7 & 19.7 & 19.6 & 28 \\
$6: 31: 00$ & 21.9 & 16.4 & 30.4 & 19.7 & 19.7 & 19.5 & 27.9 \\
$6: 31: 15$ & 21.9 & 16.4 & 30.1 & 19.7 & 19.7 & 19.5 & 27.9 \\
$6: 31: 30$ & 21.9 & 16.4 & 30.1 & 19.7 & 19.7 & 19.5 & 27.8
\end{tabular}




\section{References}

1. Le, C.; Wismer, M.K.; Shi, Z.C.; Zhang, R.; Conway, D.V.; Li, G.; Vachal, P.; Davies, I.W.; MacMillan, D.W.C., A General Small-Scale Reactor to Enable Standardization and Acceleration of Photocatalytic Reactions. ACS Cent. Sci. 2017, 3, 647-653.

2. E. B. Corcoran, M. T. Pirnot, S. Lin, S. D. Dreher, D. A. DiRocco, I. W. Davies, S. L. Buchwald, D. W. C. MacMillan, Aryl amination using ligand-free Ni(II) salts and photoredox catalysis, Science, 2016, 353, 279-283. 